Do cálculo à cohomologia: cohomologia de de Rham 
SERVIÇO DE PÓS-GRADUAÇÃO DO ICMC-USP

Data de Depósito:

Assinatura:

\section{Do cálculo à cohomologia: cohomologia de de Rham}

\section{Thaís Zanutto Mendes}

Orientador: Prof. Dr. Daniel Levcovitz

Dissertação apresentada ao Instituto de Ciências Matemáticas e de Computação - ICMC-USP, como parte dos requisitos para obtenção do título de Mestre em Ciências - Matemática . VERSÃO REVISADA

USP - São Carlos

Junho de 2012 
Ficha catalográfica elaborada pela Biblioteca Prof. Achille Bassi e Seção Técnica de Informática, ICMC/USP, com os dados fornecidos pelo(a) autor(a)

Mendes, Thaís Zanutto
Do cálculo à cohomologia: cohomologia de de Rham /
Thaís Zanutto Mendes; orientador Daniel Levcovitz. --
São Carlos, 2012.
67 p.
Dissertação (Mestrado - Programa de Pós-Graduação en
Ciências de Computação e Matemática Computacional) --
Instituto de Ciências Matemáticas e de Computação,
Universidade de São Paulo, 2012.
1. cohomologia. 2. cohomologia de de Rham. 3. II.
álgebra homológica. I. Levcovitz, Daniel, orient. II.
Título.


"Nothing in life is to be feared, it is only to be understood. Now is the time to understand more, so that we may fear less."

Marie Curie 


\section{Agradecimentos}

Ao Pai Todo Amor, por caminhar ao meu lado.

Ao meu orientador, que foi sempre um apoio por todo o caminho do mestrado.

Aos meus pais e irmãos, por todo carinho e apoio que me deram.

Ao Adriano, por acreditar sempre que eu conseguiria, mesmo quando eu mesma não acreditava. 


\section{Resumo}

Neste trabalho, estudamos a cohomologia de de Rham e métodos para os seus cálculos. Finalizamos com aplicações da cohomologia de de Rham em teoremas da topologia. 


\section{Abstract}

In this work we study the de Rham cohomology and methods for its calculations. We close it with applications of the Rham cohomology in theorems from topology. 


\section{Sumário}

Introdução 1

0.1 Aplicações Multilineares Alternadas . . . . . . . . . . . . . . . . . . 1

$0.2 \quad$ O produto exterior $\ldots \ldots \ldots \ldots \ldots \ldots \ldots \ldots$

1 A cohomologia de de Rham 13

1.1 Formas diferenciais . . . . . . . . . . . . . . . . . . . 13

1.2 A cohomologia de de Rham . . . . . . . . . . . . . . 18

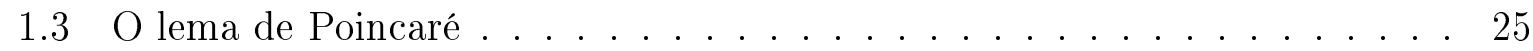

2 Complexos de cadeia e suas cohomologias 29

2.1 Complexos de Cadeia . . . . . . . . . . . . . . . . . . . . 29

2.2 Cohomologia de complexos de cadeia . . . . . . . . . . . . . 33

\begin{tabular}{|lll}
3 & A sequência de Mayer-Vietoris & 39
\end{tabular}

$\begin{array}{|ll|}4 & \text { Homotopia }\end{array}$

4.1 O funtor de cohomologia . . . . . . . . . . . . . . . . 45

5 Aplicações da Cohomologia de de Rham 55

5.1 Primeiros resultados . . . . . . . . . . . . . . . . 55 


\section{Introdução}

\subsection{Aplicações Multilineares Alternadas}

Seja $V$ um espaço vetorial sobre $\mathbb{R}$. Uma aplicação:

$$
\omega: V^{k}:=V \underbrace{\times \ldots \times}_{k \text { vezes }} V \longrightarrow \mathbb{R}
$$

é chamada $k$-linear, ou simplesmente multilinear, se for linear em cada uma de suas entradas, ou seja, se $v_{1}, v_{2}, \ldots, v_{k}, w_{i} \in V$ e $\lambda \in \mathbb{R}$, então:

$$
\omega\left(v_{1}, \ldots, \lambda v_{i}+w_{i}, \ldots, v_{k}\right)=\lambda \omega\left(v_{1}, \ldots, v_{i}, \ldots, v_{k}\right)+\omega\left(v_{1}, \ldots, w_{i}, \ldots, v_{k}\right) .
$$

Definição 0.1.1. Uma aplicação k-linear $\omega: V^{k} \longrightarrow \mathbb{R}$ é dita alternada se $\omega\left(\xi_{1}, \ldots, \xi_{k}\right)=$ 0 , sempre que $\xi_{i}=\xi_{j}$ para algum par $i \neq j$. O espaço vetorial das aplicações $k$-lineares alternadas será denotado por $A_{l t}^{k}(V)$.

Notemos que $\operatorname{Alt}^{k}(V)=0$ sempre que $k>\operatorname{dim} V$. De fato, se tomarmos $\xi_{1}, \ldots, \xi_{k} \in V$, como $k>\operatorname{dim} V$, existe pelo menos um vetor $\xi_{i}$ que é combinação linear dos outros vetores, ou seja, $\xi_{i}=\sum_{j=1, j \neq i}^{k} \lambda_{j} \xi_{j}$. Assim:

$$
\begin{aligned}
\omega\left(\xi_{1}, \ldots, \xi_{i}, \ldots \xi_{k}\right) & =\omega\left(\xi_{1}, \ldots, \sum_{j=1, j \neq i}^{k} \lambda_{j} \xi_{j}, \ldots \xi_{k}\right) \\
& =\sum_{j=1, j \neq i}^{k} \lambda_{j} \omega\left(\xi_{1}, \ldots, \xi_{j}, \ldots \xi_{k}\right)=0,
\end{aligned}
$$


pois $\omega$ é alternada. Isso também nos diz que se $\omega$ é uma aplicação $k$-linear alternada e $\xi_{1}, \ldots, \xi_{k} \in V$ são linearmente dependentes então $\omega\left(\xi_{1}, \ldots, \xi_{k}\right)=0$. Logo, para saber se um conjunto de $k$ vetores são linearmente independentes, basta encontrar uma aplicação $k$-linear alternada tal que $\omega\left(\xi_{1}, \ldots, \xi_{k}\right) \neq 0$.

O grupo simétrico de permutações do conjunto $\{1,2, \ldots, k\}$ é denotado por $S(k)$. Lembramos ainda que qualquer permutação pode ser escrita como uma composição de transposições (usualmente dizemos produto de transposições). A transposição que leva $i$ em $j$ e fixa os demais elementos será denotada por $(i, j)$. O sinal da permutação:

$$
\text { sign }: S(k) \longrightarrow\{ \pm 1\}
$$

é um homomorfismo; $\operatorname{sign}(\sigma \tau)=\operatorname{sign}(\sigma) \circ \operatorname{sign}(\tau)=\operatorname{sign}(\sigma) \cdot \operatorname{sign}(\tau)$, que aplica toda transposição em -1. Assim, o sinal de $\sigma \in S(k)$ é -1 se $\sigma$ se decompõe em um produto de uma quantidade ímpar de transposições e é 1 caso contrário.

Lema 0.1.2. Se $\omega \in A l t^{k}(V)$ e $\sigma \in S(k)$, então:

$$
\omega\left(\xi_{\sigma(1)}, \ldots, \xi_{\sigma(k)}\right)=\operatorname{sign}(\sigma) \cdot \omega\left(\xi_{1}, \ldots, \xi_{k}\right)
$$

Demonstração: Como toda permutação pode ser escrita como produto de transposições, é suficiente provar para $\sigma=(i, j)$. Para simplificar, escreveremos:

$$
\omega_{i, j}\left(\xi, \xi^{\prime}\right)=\omega\left(\xi_{1}, \ldots, \xi, \ldots, \xi^{\prime}, \ldots, \xi_{k}\right)
$$

onde $\xi$ e $\xi^{\prime}$ ocupam respectivamente as coordenadas $i$ e $j$. O restante dos vetores são escolhidos arbitrariamente, mas fixados. Segue da definição que $\omega_{i, j} \in \operatorname{Alt}^{2}(V)$. Então $\omega_{i, j}\left(\xi+\xi^{\prime}, \xi+\xi^{\prime}\right)=0$. Assim:

$$
\begin{gathered}
0=\omega_{i, j}\left(\xi+\xi^{\prime}, \xi+\xi^{\prime}\right)=\omega_{i, j}(\xi, \xi)+\omega_{i, j}\left(\xi, \xi^{\prime}\right)+\omega_{i, j}\left(\xi^{\prime}, \xi\right)+\omega_{i, j}\left(\xi^{\prime}, \xi^{\prime}\right) \\
0=\omega_{i, j}\left(\xi, \xi^{\prime}\right)+\omega_{i, j}\left(\xi^{\prime}, \xi\right) \Leftrightarrow \omega_{i, j}\left(\xi, \xi^{\prime}\right)=-\omega_{i, j}\left(\xi^{\prime}, \xi\right),
\end{gathered}
$$

o que encerra a nossa demonstração.

O que provamos acima também mostra que se uma aplicação é $k$-linear e alternada, então ela é anti-simétrica. A inversa dessa afirmação também é verdadeira, ou seja, se $\omega$ é anti-simétrica, então ela é alternada. Ora, se $\omega_{i, j}$ é anti-simétrica, $\omega_{i, j}(\xi, \xi)=-\omega_{i, j}(\xi, \xi)$ e assim $2 \omega_{i, j}(\xi, \xi)=0$, e portanto $\omega_{i, j}(\xi, \xi)=0$. 
Exemplo 0.1.3. Seja $V=\mathbb{R}^{k}$ e $\xi_{i}=\left(\xi_{i 1}, \ldots, \xi_{i k}\right)$. A função $\omega\left(\xi_{1}, \ldots, \xi_{k}\right)=\operatorname{det}\left(\xi_{i j}\right) \dot{e}$ alternada. De acordo com as propriedades de determinante, $\omega$ é anti-simétrica, e portanto, alternada.

Exemplo 0.1.4. A aplicação bilinear $\omega\left(\xi_{1}, \xi_{2}\right)=\xi_{1}^{1} \xi_{2}^{2}-\xi_{2}^{1} \xi_{1}^{2}$, onde $\xi_{1}=\left(\xi_{1}^{1}, \xi_{1}^{2}\right)$ e $\xi_{2}=$ $\left(\xi_{2}^{1}, \xi_{2}^{2}\right)$ é alternada.

\section{$0.2 \mathrm{O}$ produto exterior}

A partir de agora definiremos o produto exterior, que é uma função de $\operatorname{Alt}^{p}(V) \times$ $\operatorname{Alt}^{q}(V)$ em $\operatorname{Alt}^{p+q}(V)$.

Definição 0.2.1. Um $(p, q)$-embaralhamento $\sigma$ é uma permutação de $I_{p+q}=\{1, \ldots, p+q\}$ satisfazendo:

$$
\sigma(1)<\sigma(2)<\ldots<\sigma(p) \quad \text { e } \quad \sigma(p+1)<\ldots \sigma(p+q)
$$

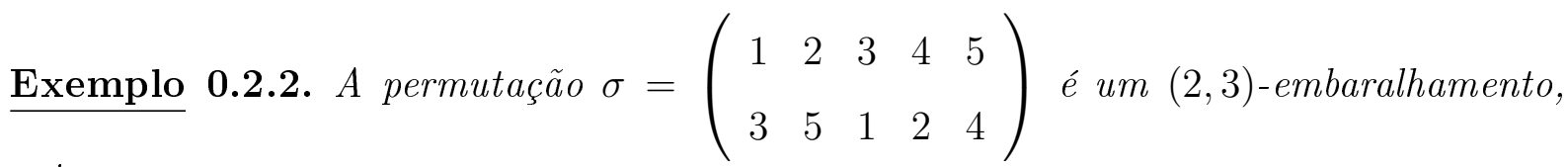
pois:

$$
\sigma(1)=3<\sigma(2)=5 \quad \text { e } \quad \sigma(3)=1<\sigma(4)=2<\sigma(5)=4 .
$$

$\operatorname{Um}(p, q)$-embaralhamento permuta o conjunto $I_{p+q}$, tomando $p$ primeiros elementos e os dispondo de forma crescente. Então, a imagem dos outros $q$ elementos fica completamente determinada. Logo a quantidade de $(p, q)$-embaralhamentos diferentes é a quantidade de combinações dos $p+q$ elementos de $I_{p+q} p$ a $p$. Assim, o conjunto de todos os $(p, q)$-embaralhamentos tem $\left(\begin{array}{c}p+q \\ p\end{array}\right)$ elementos. Denotaremos esse conjunto por $S(p, q)$.

Definição 0.2.3. Sejam $\omega_{1} \in A l t^{p}(V)$ e $\omega_{2} \in A l t^{q}(V)$, chamamos a aplicação:

$$
\left(\omega_{1} \wedge \omega_{2}\right)\left(\xi_{1}, \ldots, \xi_{p+q}\right)=\sum_{\sigma \in S(p, q)} \operatorname{sign}(\sigma) \cdot \omega_{1}\left(\xi_{\sigma(1)}, \ldots, \xi_{\sigma(p)}\right) \cdot \omega_{2}\left(\xi_{\sigma(p+1)}, \ldots, \xi_{\sigma(p+q)}\right)
$$

onde $\xi_{1}, \ldots, \xi_{p+q} \in V$, de produto exterior de $\omega_{1}$ e $\omega_{2}$. 
Exemplo 0.2.4. Quando $p=q=1$, o produto exterior é dado por $\left(\omega_{1} \wedge \omega_{2}\right)\left(\xi_{1}, \xi_{2}\right)=$ $\omega_{1}\left(\xi_{1}\right) \cdot \omega_{2}\left(\xi_{2}\right)-\omega_{1}\left(\xi_{2}\right) \cdot \omega_{2}\left(\xi_{1}\right)$, onde $\omega_{1}, \omega_{2} \in A l t^{1}(V)$ e $\xi_{1}, \xi_{2} \in V$.

Exemplo 0.2.5. Tomemos as seguintes aplicações alternadas:

$$
\begin{aligned}
\omega_{1}: \mathbb{R}^{2} \times \mathbb{R}^{2} & \longrightarrow \mathbb{R} & \omega_{2}: \mathbb{R}^{2} & \longrightarrow \mathbb{R} \\
\left(\xi_{1}, \xi_{2}\right) & \longmapsto \xi_{1}^{1} \xi_{2}^{2}-\xi_{1}^{2} \xi_{2}^{1} & \left(\xi_{1}^{1}, \xi_{1}^{2}\right) & \longmapsto \xi_{1}^{1} \xi_{1}^{2}
\end{aligned}
$$

onde $\xi_{1}=\left(\xi_{1}^{1}, \xi_{1}^{2}\right)$ e $\xi_{2}=\left(\xi_{2}^{1}, \xi_{2}^{2}\right)$. Sabemos que existem três $(2,1)$-embaralhamentos, pois $\left(\begin{array}{l}3 \\ 2\end{array}\right)=3$. São eles:

$$
\sigma_{1}=\left(\begin{array}{lll}
1 & 2 & 3 \\
1 & 2 & 3
\end{array}\right) \quad ; \quad \sigma_{2}=\left(\begin{array}{lll}
1 & 2 & 3 \\
1 & 3 & 2
\end{array}\right) \quad \text { e } \sigma_{3}\left(\begin{array}{lll}
1 & 2 & 3 \\
2 & 3 & 1
\end{array}\right)
$$

com $\operatorname{sign}\left(\sigma_{1}\right)=1, \operatorname{sign}\left(\sigma_{2}\right)=-1$ e $\operatorname{sign}\left(\sigma_{3}\right)=1$. Assim:

$$
\begin{aligned}
\left(\omega_{1} \wedge \omega_{2}\right)\left(\xi_{1}, \xi_{2}, \xi_{3}\right) & =\sum_{\sigma \in S(2,1)} \operatorname{sign}(\sigma) \cdot \omega_{1}\left(\xi_{\sigma(1)}, \xi_{\sigma(2)}\right) \cdot \omega_{2}\left(\xi_{\sigma(3)}\right)= \\
& =\operatorname{sign}\left(\sigma_{1}\right) \omega_{1}\left(\xi_{1}, \xi_{2}\right) \omega_{2}\left(\xi_{3}\right)+\operatorname{sign}\left(\sigma_{2}\right) \omega_{1}\left(\xi_{1}, \xi_{3}\right) \omega_{2}\left(\xi_{2}\right)+\operatorname{sign}\left(\sigma_{3}\right) \omega_{1}\left(\xi_{2}, \xi_{3}\right) \omega_{2}\left(\xi_{1}\right)= \\
& =\left(\xi_{1}^{1} \xi_{2}^{2}-\xi_{1}^{2} \xi_{2}^{1}\right) \xi_{3}^{1} \xi_{3}^{2}-\left(\xi_{1}^{1} \xi_{3}^{2}-\xi_{1}^{2} \xi_{3}^{1}\right) \xi_{2}^{1} \xi_{2}^{2}+\left(\xi_{2}^{1} \xi_{3}^{2}-\xi_{2}^{2} \xi_{3}^{1}\right) \xi_{1}^{1} \xi_{1}^{2} .
\end{aligned}
$$

O produto exterior $\omega_{1} \wedge \omega_{2}$ é uma forma $(p+q)$-linear, pois $\omega_{1}$ e $\omega_{2}$ são $p$-linear e $q$-linear, respectivamente. Além disso:

Lema 0.2.6. Se $\omega_{1} \in A l t^{p}(V)$ e $\omega_{2} \in A l t^{q}(V)$. Entãa $\omega_{1} \wedge \omega_{2} \in A l t^{p+q}(V)$.

Para essa demonstração é necessário antes demonstrar outro lema:

Lema 0.2.7. Uma aplicação k-linear $\omega$ é alternada se $\omega\left(\xi_{1}, \ldots, \xi_{k}\right)=0$ para toda $k$-upla $\operatorname{com} \xi_{i}=\xi_{i+1}$, para algum $1 \leqslant i \leqslant k-1$.

Demonstração: Temos que $S(k)$ é gerado por transposições do tipo $(i, i+1)$ e, pelo mesmo argumento usado no lema 0.1 .2 , temos:

$$
\omega\left(\xi_{1}, \ldots, \xi_{i}, \xi_{i+1}, \ldots, \xi_{k}\right)=-\omega\left(\xi_{1}, \ldots, \xi_{i+1}, \xi_{i}, \ldots, \xi_{k}\right)
$$


Como o lema 0.1 .2 é válido para toda $\sigma \in S(k)$, então, $\omega$ é alternada.

Agora munidos desse resultado, voltemos nossa atenção a demonstração desejada:

Demonstração do lema 0.2.6 Mostremos primeiramente que $\omega_{1} \wedge \omega_{2}\left(\xi_{1}, \ldots, \xi_{p+q}\right)=0$ quando $\xi_{1}=\xi_{2}$. Façamos:

(i) $S_{12}=\{\sigma \in S(p, q) / \sigma(1)=1, \sigma(p+1)=2\}$;

(ii) $S_{21}=\{\sigma \in S(p, q) / \sigma(1)=2, \sigma(p+1)=1\}$;

(iii) $S_{\circ}=S(p, q)-\left(S_{12} \bigcup S_{21}\right)$.

Caso $\sigma \in S_{\circ}$ então $\omega_{1}\left(\xi_{\sigma(1)}, \ldots, \xi_{\sigma(p)}\right)=0$ ou $\omega_{2}\left(\xi_{\sigma(p+1)}, \ldots, \xi_{\sigma(p+q)}\right)=0$, já que $\xi_{\sigma(1)}=\xi_{\sigma(2)}$ ou $\xi_{\sigma(p+1)}=\xi_{\sigma(p+2)}$. Notemos ainda que $S_{12}$ é aplicado em $S_{21}$ de forma bijetiva compondo $\tau=(1,2)$ com cada permutação de $S_{12}$. Assim, temos:

$$
\begin{aligned}
\omega_{1} \wedge \omega_{2}\left(\xi_{1}, \ldots, \xi_{p+q}\right)= & \sum_{\sigma \in S(p, q)} \operatorname{sign}(\sigma) \cdot \omega_{1}\left(\xi_{\sigma(1)}, \ldots, \xi_{\sigma(p)}\right) \cdot \omega_{2}\left(\xi_{\sigma(p+1)}, \ldots, \xi_{\sigma(p+q)}\right) \\
= & \sum_{\sigma \in S_{12}} \operatorname{sign}(\sigma) \cdot \omega_{1}\left(\xi_{\sigma(1)}, \ldots, \xi_{\sigma(p)}\right) \cdot \omega_{2}\left(\xi_{\sigma(p+1)}, \ldots, \xi_{\sigma(p+q)}\right) \\
& +\sum_{\sigma \in S_{21}} \operatorname{sign}(\sigma) \cdot \omega_{1}\left(\xi_{\sigma(1)}, \ldots, \xi_{\sigma(p)}\right) \cdot \omega_{2}\left(\xi_{\sigma(p+1)}, \ldots, \xi_{\sigma(p+q)}\right) \\
& +\sum_{\sigma \in S_{\circ}} \operatorname{sign}(\sigma) \cdot \omega_{1}\left(\xi_{\sigma(1)}, \ldots, \xi_{\sigma(p)}\right) \cdot \omega_{2}\left(\xi_{\sigma(p+1)}, \ldots, \xi_{\sigma(p+q)}\right) \\
= & \sum_{\sigma \in S_{12}} \operatorname{sign}(\sigma) \cdot \omega_{1}\left(\xi_{\sigma(1)}, \ldots, \xi_{\sigma(p)}\right) \cdot \omega_{2}\left(\xi_{\sigma(p+1)}, \ldots, \xi_{\sigma(p+q)}\right)+ \\
& +\sum_{\sigma \in S_{12}} \operatorname{sign}(\tau \sigma) \cdot \omega_{1}\left(\xi_{\tau \sigma(1)}, \ldots, \xi_{\tau \sigma(p)}\right) \cdot \omega_{2}\left(\xi_{\tau \sigma(p+1)}, \ldots, \xi_{\tau \sigma(p+q)}\right) \\
= & \sum_{\sigma \in S_{12}} \operatorname{sign}(\sigma) \cdot \omega_{1}\left(\xi_{\sigma(1)}, \ldots, \xi_{\sigma(p)}\right) \cdot \omega_{2}\left(\xi_{\sigma(p+1)}, \ldots, \xi_{\sigma(p+q)}\right) \\
& -\sum_{\sigma \in S_{12}} \operatorname{sign}(\sigma) \cdot \omega_{1}\left(\xi_{\tau \sigma(1)}, \ldots, \xi_{\tau \sigma(p)}\right) \cdot \omega_{2}\left(\xi_{\tau \sigma(p+1)}, \ldots, \xi_{\tau \sigma(p+q)}\right) .
\end{aligned}
$$

Desde que $\sigma(1)=1$ e $\sigma(p+1)=2$, temos $\tau \sigma(1)=2$ e $\tau \sigma(p+1)=1$ e vemos $\tau \sigma(i)=i$, sempre que $i \neq 1$ ou $i \neq p+1$ ). Como $\xi_{1}=\xi_{2}$, os termos das duas somas se cancelam.

Para o caso geral $\xi_{i}=\xi_{i+1}$, o raciocínio é mesmo. Consideremos:

(i) $S_{i+1}=\{\sigma \in S(p, q) / \sigma(i) \neq r, r \geqslant p+1 ; \sigma(p+1) \neq s, s \leqslant p\}$;

(ii) $S_{i+1} i=\{\sigma \in S(p, q) / \sigma(i) \neq r, r \leqslant p ; \sigma(p+1) \neq s, s \geqslant p+1\}$;

(iii) $S_{\circ}=S(p, q)-\left(S_{i i+1} \bigcup S_{i+1} i\right)$. 
Assim, para $\sigma \in S_{\circ}$, temos $\omega_{1}\left(\xi_{\sigma(1)}, \ldots, \xi_{\sigma(p)}\right)=0$ ou $\omega_{2}\left(\xi_{\sigma(p+1)}, \ldots, \xi_{\sigma(p+q)}\right)=0$. Então:

$$
\begin{aligned}
\omega_{1} \wedge \omega_{2}\left(\xi_{1}, \ldots, \xi_{p+q}\right)= & \sum_{\sigma \in S_{i}} \operatorname{sign}(\sigma) \cdot \omega_{1}\left(\xi_{\sigma(1)}, \ldots, \xi_{\sigma(p)}\right) \cdot \omega_{2}\left(\xi_{\sigma(p+1)}, \ldots, \xi_{\sigma(p+q)}\right) \\
& +\sum_{\sigma \in S_{i+1} i} \operatorname{sign}(\sigma) \cdot \omega_{1}\left(\xi_{\sigma(1)}, \ldots, \xi_{\sigma(p)}\right) \cdot \omega_{2}\left(\xi_{\sigma(p+1)}, \ldots, \xi_{\sigma(p+q)}\right) .
\end{aligned}
$$

Usando agora a transposição $\tau=(i, i+1)$, temos uma bijeção de $S_{i{ }_{i+1}}$ em $S_{i+1}{ }_{i}$ e, como antes, a soma se cancelará. Logo:

$$
\omega_{1} \wedge \omega_{2}\left(\xi_{1}, \ldots, \xi_{p+q}\right)=0
$$

sempre que $\xi_{i}=\xi_{i+1}$, para algum $1 \leqslant i \leqslant k-1$ na $(p+q)$-upla. Então, pelo lema 0.2.6. $\omega_{1} \wedge \omega_{2} \in \operatorname{Alt}^{p+q}(V)$.

Enunciaremos alguma propriedades do produto exterior. Consideremos $\omega_{1}, \omega_{1}^{\prime} \in \operatorname{Alt}^{p}(V)$, $\omega_{2}, \omega_{2}^{\prime} \in \operatorname{Alt}^{q}(V)$ e $\lambda \in \mathbb{R}$. Então:

1. $\left(\omega_{1}+\omega_{1}^{\prime}\right) \wedge \omega_{2}=\omega_{1} \wedge \omega_{2}+\omega_{1}^{\prime} \wedge \omega_{2}$;

2. $\left(\lambda \omega_{1}\right) \wedge \omega_{2}=\lambda\left(\omega_{1} \wedge \omega_{2}\right)=\omega_{1} \wedge\left(\lambda \omega_{2}\right)$;

3. $\omega_{1} \wedge\left(\omega_{2}+\omega_{2}^{\prime}\right)=\omega_{1} \wedge \omega_{2}+\omega_{1} \wedge \omega_{2}^{\prime}$.

Lema 0.2.8. Se $\omega_{1} \in A l t^{p}(V)$ e $\omega_{2} \in A l t^{q}(V)$, então $\omega_{1} \wedge \omega_{2}=(-1)^{p q} \omega_{2} \wedge \omega_{1}$.

Demonstração: Seja $\theta \in S(p+q)$ tal que:

$$
\begin{aligned}
& \theta(1)=p+1, \theta(2)=p+2, \ldots, \theta(q)=p+q \\
& \theta(q+1)=1, \theta(q+2)=2, \ldots, \theta(p+q)=p .
\end{aligned}
$$

Temos que $\operatorname{sign}(\theta)=(-1)^{p q}$. Não é difícil ver que a composição de $\theta$ com os elementos de $S(p, q)$ gera uma bijeção:

$$
\begin{aligned}
S(p, q) & \longleftrightarrow S(q, p) \\
\sigma & \longmapsto \sigma \circ \theta,
\end{aligned}
$$

ou seja, essa bijeção simplesmentes inverte a ordem do embaralhamento. 
Observemos que:

$$
\begin{aligned}
\omega_{2}\left(\xi_{\sigma \theta(1)}, \ldots, \xi_{\sigma \theta(q)}\right) & =\omega_{2}\left(\xi_{\sigma(p+1)}, \ldots, \xi_{\sigma(p+q)}\right) \\
\omega_{1}\left(\xi_{\sigma \theta(q+1)}, \ldots, \xi_{\sigma \theta(p+q)}\right) & =\omega_{1}\left(\xi_{\sigma(1)}, \ldots, \xi_{\sigma(p)}\right) .
\end{aligned}
$$

Então:

$$
\begin{aligned}
\omega_{2} \wedge \omega_{1}\left(\xi_{1}, \ldots, \xi_{p+q}\right) & =\sum_{\sigma \in S(p, q)} \operatorname{sign}(\sigma) \cdot \omega_{2}\left(\xi_{\sigma(1)}, \ldots, \xi_{\sigma(q)}\right) \cdot \omega_{1}\left(\xi_{\sigma(q+1)}, \ldots, \xi_{\sigma(p+q)}\right) \\
& =\sum_{\sigma \in S(p, q)} \operatorname{sign}(\sigma \theta) \cdot \omega_{2}\left(\xi_{\sigma \theta(1)}, \ldots, \xi_{\sigma \theta(q)}\right) \cdot \omega_{1}\left(\xi_{\sigma \theta(q+1)}, \ldots, \xi_{\sigma \theta(p+q)}\right) \\
& =(-1)^{p q} \sum_{\sigma \in S(p, q)} \operatorname{sign}(\sigma) \cdot \omega_{1}\left(\xi_{\sigma(1)}, \ldots, \xi_{\sigma(p)}\right) \cdot \omega_{2}\left(\xi_{\sigma(p+1)}, \ldots, \xi_{\sigma(p+q)}\right) \\
& =(-1)^{p q} \omega_{1} \wedge \omega_{2}\left(\xi_{1}, \ldots, \xi_{p+q}\right) .
\end{aligned}
$$

Lema 0.2.9. Se $\omega \in A l t^{p}(V), \omega_{2} \in A l t^{q}(V)$ e $\omega_{3} \in A l t^{r}(V)$, então:

$$
\omega_{1} \wedge\left(\omega_{2} \wedge \omega_{3}\right)=\left(\omega_{1} \wedge \omega_{2}\right) \wedge \omega_{3}
$$

Demonstração: Seja $S(p, q, r) \subset S(p+q+r)$ o conjunto das permutações que satisfazem:

$$
\begin{aligned}
\sigma(1) & <\ldots<\sigma(p) \\
\sigma(p+1) & <\ldots<\sigma(p+q) \\
\sigma(p+q+1) & <\ldots<\sigma(p+q+r) .
\end{aligned}
$$

Consideremos os seguintes subconjuntos de $S(p, q, r)$ :

$$
\begin{aligned}
& S(\bar{p}, q, r)=\{\sigma \in S(p, q, r) / \sigma(i)=i, 1 \leqslant i \leqslant p\} \\
& S(p, q, \bar{r})=\{\sigma \in S(p, q, r) / \sigma(i)=i, p+q+1 \leqslant i \leqslant p+q+r\} .
\end{aligned}
$$

Consideremos a seguinte bijeção:

$$
\begin{aligned}
S(p, q+r) \times S(\bar{p}, q, r) & \longleftrightarrow S(p, q, r) \\
(\sigma, \theta) & \longmapsto \sigma \circ \theta .
\end{aligned}
$$


Com todas essas informações:

$$
\begin{aligned}
& {\left[\omega_{1} \wedge\left(\omega_{2} \wedge \omega_{3}\right)\right]\left(\xi_{1}, \ldots, \xi_{p+q+r}\right)=\sum_{\sigma \in S(p, q+r)} \operatorname{sign}(\sigma) \cdot \omega_{1}\left(\xi_{\sigma(1)}, \ldots, \xi_{\sigma(p)}\right)\left(\omega_{2} \wedge \omega_{3}\right)\left(\xi_{\sigma(p+1)}, \ldots, \xi_{\sigma(p+q+r)}\right)} \\
& =\sum_{\sigma \in S(p, q+r)} \operatorname{sign}(\sigma) \sum_{\theta \in S(\bar{p}, q, r)} \operatorname{sign}(\theta)\left[\omega _ { 1 } ( \xi _ { \sigma ( 1 ) } , \ldots , \xi _ { \sigma ( p ) } ) \omega _ { 2 } ( \xi _ { \sigma \theta ( p + 1 ) } , \ldots , \xi _ { \sigma \theta ( p + q ) } ) \omega _ { 3 } \left(\xi_{\sigma \theta(p+q+1)}, \ldots, \xi_{\sigma \theta(p+q+r}\right.\right. \\
& =\sum_{\substack{\sigma \in S(p, q+r) \\
\theta \in S(\bar{p}, q, r)}} \operatorname{sign}(\sigma \theta)\left[\omega_{1}\left(\xi_{\sigma \theta(1)}, \ldots, \xi_{\sigma \theta(p)}\right) \omega_{2}\left(\xi_{\sigma \theta(p+1)}, \ldots, \xi_{\sigma \theta(p+q)}\right) \omega_{3}\left(\xi_{\sigma \theta(p+q+1)}, \ldots, \xi_{\sigma \theta(p+q+r)}\right)\right] \\
& =\sum_{\mu \in S(p, q, r)} \operatorname{sign}(\sigma \theta)\left[\omega_{1}\left(\xi_{\mu(1)}, \ldots, \xi_{\mu(p)}\right) \omega_{2}\left(\xi_{\mu(p+1)}, \ldots, \xi_{\mu(p+q)}\right) \omega_{3}\left(\xi_{\mu(p+q+1)}, \ldots, \xi_{\mu(p+q+r)}\right)\right]
\end{aligned}
$$

Onde usamos que $\sigma(i)=\sigma \theta(i), 1 \leqslant i \leqslant p$ e a bijeção 0.2 .1 na última passagem. Isso porque a combinação dos dois somatórios, descrita na terceira linha, gera todas as combinações possíveis, logo, a troca é possível.

Consideremos agora a seguinte bijeção:

$$
\begin{aligned}
S(p+q, r) \times S(p, q, \bar{r}) & \longleftrightarrow S(p, q, r) \\
(\eta, \mu) & \longmapsto \eta \circ \mu .
\end{aligned}
$$

Analogamente, teremos:

$$
\begin{aligned}
& {\left[\left(\omega_{1} \wedge \omega_{2}\right) \wedge \omega_{3}\right]\left(\xi_{1}, \ldots, \xi_{p+q+r}\right)=\sum_{\eta \in S(p+q, r)} \operatorname{sign}(\eta) \cdot\left(\omega_{1} \wedge \omega_{2}\right)\left(\xi_{\eta(1)}, \ldots, \xi_{\eta(p+q)}\right) \omega_{3}\left(\xi_{\eta(p+q+1)}, \ldots, \xi_{\eta(p+q+r)}\right.} \\
& =\sum_{\eta \in S(p+q, r)} \operatorname{sign}(\eta) \sum_{\mu \in S(p, q, \bar{r})} \operatorname{sign}(\mu)\left[\omega _ { 1 } ( \xi _ { \eta \mu ( 1 ) } , \ldots , \xi _ { \eta \mu ( p ) } ) \omega _ { 2 } ( \xi _ { \eta \mu ( p + 1 ) } , \ldots , \xi _ { \eta \mu ( p + q ) } ) \omega _ { 3 } \left(\xi_{\eta(p+q+1)}, \ldots, \xi_{\eta(p+q+r}\right.\right. \\
& =\sum_{\substack{\eta \in S(p+q, r) \\
\mu \in S(p, q, \bar{r})}} \operatorname{sign}(\eta \mu)\left[\omega_{1}\left(\xi_{\eta \mu(1)}, \ldots, \xi_{\eta \mu(p)}\right) \omega_{2}\left(\xi_{\eta \mu(p+1)}, \ldots, \xi_{\eta \mu(p+q)}\right) \omega_{3}\left(\xi_{\eta \mu(p+q+1)}, \ldots, \xi_{\eta \mu(p+q+r)}\right)\right] \\
& =\sum_{v \in S(p, q, r)} \operatorname{sign}(\eta \mu)\left[\omega_{1}\left(\xi_{v(1)}, \ldots, \xi_{v(p)}\right) \omega_{2}\left(\xi_{v(p+1)}, \ldots, \xi_{v(p+q)}\right) \omega_{3}\left(\xi_{v(p+q+1)}, \ldots, \xi_{v(p+q+r)}\right)\right] .
\end{aligned}
$$

Onde usamos que $\eta(i)=\eta \mu(i), p+q+1 \leqslant i \leqslant p+q+r$ e a bijeção 0.2 .2 para a última passagem, como feito antes.

Logo, $\omega_{1} \wedge\left(\omega_{2} \wedge \omega_{3}\right)=\left(\omega_{1} \wedge \omega_{2}\right) \wedge \omega_{3}$ como queríamos demonstrar.

Observação 0.2.10. É comum encontrarmos na literatura a seguinte definição:

$$
\omega_{1} \bar{\wedge} \omega_{2}\left(\xi_{1}, \ldots, \xi_{p+q}\right)=\frac{1}{p ! q !} \sum_{\sigma \in S(p+q)} \operatorname{sign}(\sigma) \cdot \omega_{2}\left(\xi_{\sigma(1)}, \ldots, \xi_{\sigma(q)}\right) \cdot \omega_{1}\left(\xi_{\sigma(q+1)}, \ldots, \xi_{\sigma(p+q)}\right)
$$


Note que nessa definiçãa $\{\sigma(1), \ldots, \sigma(p)\}$ e $\{\sigma(p+1), \ldots, \sigma(p+q)\}$ não estão ordenados. Ora, há exatamente $S(p) \times S(q)$ modos para que os conjuntos acima se tornem uma sequência arbitrária, e essa é a causa do fator de correção $\frac{1}{p ! q !}$ aparecer na expressão. Então $\omega_{1} \wedge \omega_{2}=\omega_{1} \bar{\wedge} \omega_{2}$.

Uma $\mathbb{R}$-álgebra $\mathcal{A}$ consiste de um espaço vetorial sobre $\mathbb{R}$ e uma aplicação bilinear $\mu: A \times A \longrightarrow A$ que seja associativa, ou seja, $\mu(a, \mu(b, c))=\mu(\mu(a, b), c)$, para quaisquer $a, b, c \in A$. A álgebra é chamada com unidade se existir um elemento $1 \in A$ tal que $\mu(1, a)=\mu(a, 1)=a$, para todo $a \in A$. Tal elemento é chamado de unidade.

Definição 0.2.11. (i) Uma $\mathbb{R}$-álgebra graduada $\mathcal{A}_{*}$ é uma sequência de espaços vetoriais $A_{k}, k=0,1, \ldots$, e aplicações bilineares $\mu: A_{k} \times A_{l} \longrightarrow A_{k+l}$ que são associativas;

(ii) A álgebra graduada $\mathcal{A}_{*}$ é dita conexa se ela tiver unidade $1 \in A_{0}$ e se $\epsilon: \mathbb{R} \longrightarrow A_{0}$ dada por $\epsilon(r)=r \cdot 1$ é um isomorfismo;

(iii) A álgebra graduada $\mathcal{A}_{*}$ é chamada comutativa (ou anti-comutativa), se $\mu(a, b)=(-1)^{k l} \mu(b, a)$, para $a \in A_{k}$ e $b \in A_{l}$.

Os elementos de $A_{k}$ são ditos de grau $k$. Sabemos que $\operatorname{Alt}^{k}(V)$ é um espaço vetorial sobre $\mathbb{R}$ do modo usual:

$$
\begin{aligned}
\left(\omega_{1}+\omega_{2}\right)\left(\xi_{1}, \ldots, \xi_{k}\right) & =\omega_{1}\left(\xi_{1}, \ldots, \xi_{k}\right)+\omega_{2}\left(\xi_{1}, \ldots, \xi_{k}\right) \\
(\lambda \omega)\left(\xi_{1}, \ldots, \xi_{k}\right) & =\lambda\left(\omega\left(\xi_{1}, \ldots, \xi_{k}\right)\right) .
\end{aligned}
$$

Também já foi mostrado que o produro exterior é uma aplicação bilinear de $\operatorname{Alt}^{p}(V) \times$ $\operatorname{Alt}^{q}(V)$ em $\operatorname{Alt}^{p+q}(V)$. Partindo de $\operatorname{Alt}^{0}(V)=\mathbb{R}$, expandimos o produto exterior para $\operatorname{Alt}^{0}(V) \times \operatorname{Alt}^{p}(V)$ usando a estrutura de espaço vetorial. As propriedades das formas alternadas podem ser resumidas em:

Teorema 0.2.12. Alt* $(V)$ é uma álgebra graduada, anti-comutativa e conexa. 
Lema 0.2.13. Para 1 -formas $\omega_{1}, \ldots, \omega_{p} \in A l t^{1}(V)$ temos que:

$$
\omega_{1} \wedge \ldots \wedge \omega_{p}\left(\xi_{1}, \ldots, \xi_{p}\right)=\operatorname{det}\left(\begin{array}{cccc}
\omega_{1}\left(\xi_{1}\right) & \omega_{1}\left(\xi_{2}\right) & \cdots & \omega_{1}\left(\xi_{p}\right) \\
\omega_{2}\left(\xi_{1}\right) & \omega_{2}\left(\xi_{2}\right) & \cdots & \omega_{2}\left(\xi_{p}\right) \\
\vdots & \vdots & \ddots & \vdots \\
\omega_{p}\left(\xi_{1}\right) & \omega_{p}\left(\xi_{2}\right) & \cdots & \omega_{p}\left(\xi_{p}\right)
\end{array}\right)
$$

Demonstração: Por indução sobre $p$. Para $p=2$, sejam $\omega_{1}, \omega_{2} \in \operatorname{Alt}^{1}(V)$. Então:

$$
\left(\omega_{1} \wedge \omega_{2}\right)\left(\xi_{1}, \xi_{2}\right)=\sum_{\sigma \in S(1,1)} \operatorname{sign}(\sigma) \omega_{1}\left(\xi_{\sigma(1)}\right) \omega_{2}\left(\xi_{\sigma(2)}\right)=\omega_{1}\left(\xi_{1}\right) \omega_{2}\left(\xi_{2}\right)-\omega_{1}\left(\xi_{2}\right) \omega_{2}\left(\xi_{1}\right)
$$

e

$$
\operatorname{det}\left(\begin{array}{ll}
\omega_{1}\left(\xi_{1}\right) & \omega_{1}\left(\xi_{2}\right) \\
\omega_{2}\left(\xi_{1}\right) & \omega_{2}\left(\xi_{2}\right)
\end{array}\right)=\omega_{1}\left(\xi_{1}\right) \omega_{2}\left(\xi_{2}\right)-\omega_{1}\left(\xi_{2}\right) \omega_{2}\left(\xi_{1}\right)
$$

o que comprova a afirmação para $p=2$.

Suponhamos que a afirmação seja verdadeira para $p-1$. Pela definição de produto exterior, temos, para $\omega_{1}, \ldots, \omega_{p} \in \operatorname{Alt}^{1}(V)$ :

$$
\begin{aligned}
\omega_{1} \wedge\left(\omega_{2} \wedge \ldots \wedge \omega_{p}\right)\left(\xi_{1}, \ldots, \xi_{p}\right) & =\sum_{\sigma \in S(1, p-1)} \operatorname{sign}(\sigma) \omega_{1}\left(\xi_{\sigma(1)}\right)\left(\omega_{2} \wedge \ldots \wedge \omega_{p}\right)\left(\xi_{\sigma(2)}, \ldots, \xi_{\sigma(p)}\right) \\
& =\sum_{j=1}^{p}(-1)^{j+1} \omega_{1}\left(\xi_{j}\right)\left(\omega_{2} \wedge \ldots \wedge \omega_{p}\right)\left(\xi_{\sigma(2)}, \ldots, \xi_{\sigma(p)}\right) \\
& =\sum_{j=1}^{p}(-1)^{j+1} \omega_{1}\left(\xi_{j}\right)\left(\omega_{2} \wedge \ldots \wedge \omega_{p}\right)\left(\xi_{1}, \ldots, \widehat{\xi}_{j}, \ldots, \xi_{p}\right),
\end{aligned}
$$

onde $\left(\xi_{1}, \ldots, \widehat{\xi}_{j}, \ldots, \xi_{p}\right)$ é uma $(p-1)$-upla que omite a variável $\xi_{j}$. Como a afirmação é válida para $p-1$, temos:

$$
\begin{aligned}
\omega_{1} \wedge\left(\omega_{2} \wedge \ldots \wedge \omega_{p}\right)\left(\xi_{1}, \ldots, \xi_{p}\right) & =\sum_{j=1}^{p}(-1)^{j+1} \omega_{1}\left(\xi_{j}\right)\left(\omega_{2} \wedge \ldots \wedge \omega_{p}\right)\left(\xi_{1}, \ldots, \widehat{\xi}_{j}, \ldots, \xi_{p}\right) \\
& =\sum_{j=1}^{p}(-1)^{j+1} \omega_{1}\left(\xi_{j}\right) \operatorname{det}\left(\begin{array}{cccc}
\omega_{2}\left(\xi_{1}\right) & \omega_{2}\left(\xi_{2}\right) & \cdots & \omega_{2}\left(\xi_{p}\right) \\
\omega_{3}\left(\xi_{1}\right) & \omega_{3}\left(\xi_{2}\right) & \cdots & \omega_{3}\left(\xi_{p}\right) \\
\vdots & \vdots & \ddots & \vdots \\
\omega_{p}\left(\xi_{1}\right) & \omega_{p}\left(\xi_{2}\right) & \cdots & \omega_{p}\left(\xi_{p}\right)
\end{array}\right) .
\end{aligned}
$$


O último termo da igualdade acima é justamente o determinante da matriz $\left(\omega_{i}\left(\xi_{j}\right)\right)$, em expansão pela $1^{\text {a }}$ linha segundo a regra de Laplace para determinantes de matrizes quadradas.

Do lema acima, temos que se as 1-formas $\omega_{1}, \ldots, \omega_{p} \in \operatorname{Alt}^{1}(V)$ são linearmente independentes, então $\omega_{1} \wedge \ldots \wedge \omega_{p} \neq 0$. De fato, podemos escolher $\xi \in V \operatorname{com} \omega_{i}\left(\xi_{j}\right)=0$ se $i \neq j$ e $\omega_{i}\left(\xi_{j}\right)=1$, para $i=j$ e teremos $\operatorname{det}\left(\omega_{i}\left(\xi_{j}\right)\right)=1$. Reciprocamente, se $\omega_{1}, \ldots, \omega_{p} \in \operatorname{Alt}^{1}(V)$ são linearmente dependentes, existirá pelo menos um $\omega_{i}$ que poderá ser expresso como combinação linear dos outros. Ou seja, $\omega_{i}=\sum_{\substack{j=1 \\ j \neq i}} r_{j} \omega_{j}$, então:

$$
\omega_{1} \wedge \ldots \wedge \omega_{i} \wedge \ldots \wedge \omega_{p}=\sum_{\substack{j=1 \\ j \neq i}} r_{j}\left(\omega_{1} \wedge \ldots \wedge \omega_{j} \wedge \ldots \wedge \omega_{p}\right)=0
$$

pois, pelo lema anterior, podemos calcular esse produto exterior pelo determinante, que sempre se anulará em cada termo do somatório, já que teremos duas linhas iguais. Essas ressalvas provam o seguinte lema:

Lema 0.2.14. Sejam $\omega_{1}, \ldots, \omega_{p} \in A l t^{1}(V)$, então $\omega_{1} \wedge \ldots \wedge \omega_{p} \neq 0$ se e somente se, $\omega_{1}, \ldots, \omega_{p}$ são linearmente independentes.

Teorema 0.2.15. Sejam $e_{1}, \ldots, e_{n}$ uma base de $V$ e $\epsilon_{1}, \ldots, \epsilon_{n}$ a base dual de $A_{l}^{1}(V)$. Então:

$$
\left\{\epsilon_{\sigma(1)} \wedge \cdots \wedge \epsilon_{\sigma(p)}\right\}_{\sigma \in S(p, n-p)}
$$

é uma base para $\operatorname{Alt}^{p}(V)$. Em particular

$$
\operatorname{dim} A l t^{p}(V)=\left(\begin{array}{c}
\operatorname{dim} V \\
p
\end{array}\right)
$$

Demonstração do teorema 0.2.15. Como $\epsilon_{i}\left(e_{j}\right)=0$ quando $i \neq j$ e $\epsilon_{i}\left(e_{i}\right)=1$, o lema 0.2 .13 nos permite caracterizar o produto exterior do enunciado como:

$$
\epsilon_{i_{1}} \wedge \ldots \wedge \epsilon_{i_{p}}\left(e_{j_{1}}, \ldots, e_{j_{p}}\right)=\left\{\begin{array}{ccc}
0 & \text { se } & \left\{i_{1}, \ldots, i_{p}\right\} \neq\left\{j_{1}, \ldots, j_{p}\right\} \\
\operatorname{sign}(\sigma) & \text { se } \quad\left\{i_{1}, \ldots, i_{p}\right\}=\left\{j_{1}, \ldots, j_{p}\right\}
\end{array}\right.
$$


onde $\sigma$ é a permutação $\sigma\left(i_{k}\right)=j_{k}$. Então, do lema 0.1 .2 e de 0.2 .3 , temos que:

$$
\omega=\sum_{S(p, n-p)} \omega\left(e_{\sigma(1)}, \ldots, e_{\sigma(p)}\right) \epsilon_{\sigma(1)} \wedge \ldots \wedge \epsilon_{\sigma(p)}
$$

para qualquer $p$-forma alternada $\omega \in \operatorname{Alt}^{p}(V)$. De fato, ao tomarmos $e_{1}, \ldots, e_{p}$ elementos da base de $V$, temos:

$$
\begin{aligned}
\sum_{S(p, n-p)} \omega\left(e_{\sigma(1)}, \ldots, e_{\sigma(p)}\right) \epsilon_{\sigma(1)} \wedge \ldots \wedge \epsilon_{\sigma(p)}\left(e_{1}, \ldots, e_{p}\right) & =\sum_{\substack{S(p, n-p) \\
1 \leqslant \sigma(i) \leqslant p \\
1 \leqslant i \leqslant p}} \omega\left(e_{\sigma(1)}, \ldots, e_{\sigma(p)}\right) \operatorname{sign}(\sigma) \\
& =\sum_{\substack{S(p, n-p) \\
1 \leqslant \sigma(i) \leqslant p \\
1 \leqslant i \leqslant p}} \operatorname{sign}(\sigma) \omega\left(e_{1}, \ldots, e_{p}\right) \operatorname{sign}(\sigma) \\
& =\omega\left(e_{1}, \ldots, e_{p}\right),
\end{aligned}
$$

pois a única permutação em $S(p, n-p)$ a atender as condições descritas no somatório da segunda linha é a identidade. Logo $\operatorname{Alt}^{p}(V)$ é gerado pelas $\epsilon_{\sigma(1)} \wedge \ldots \wedge \epsilon_{\sigma(p)}$, com $\sigma \in S(p, n-p)$.

Para a independência linear, consideremos a seguinte expressão:

$$
\sum_{S(p, n-p)} \lambda_{\sigma} \epsilon_{\sigma(1)} \wedge \ldots \wedge \epsilon_{\sigma(p)}=0, \lambda_{\sigma} \in \mathbb{R}
$$

Como anteriormente, tomemos $e_{1}, \ldots, e_{p}$ base de $V$. Então:

$$
\sum_{S(p, n-p)} \lambda_{\sigma} \epsilon_{\sigma(1)} \wedge \ldots \wedge \epsilon_{\sigma(p)}\left(e_{1}, \ldots, e_{p}\right)=\sum_{\substack{S(p, n-p) \\ 1 \leqslant \sigma(i) \leqslant p \\ 1 \leqslant i \leqslant p}} \lambda_{\sigma} \operatorname{sign}(\sigma)=\lambda_{\sigma} .
$$

Assim, para que a expressão 0.2 .4 seja satisfeita devemos ter $\lambda_{\sigma}=0$. Logo, provamos a independência linear dos termos $\epsilon_{\sigma(1)} \wedge \ldots \wedge \epsilon_{\sigma(p)}$, com $\sigma \in S(p, n-p)$.

Logo, a dimensão de $\operatorname{Alt}^{p}(V)$ é justamente a quantidade de $(p, n-p)$-embaralhamentos existentes, ou seja, $\left(\begin{array}{c}n \\ p\end{array}\right)$ ou $\left(\begin{array}{c}\operatorname{dim} V \\ p\end{array}\right)$. O que conclui a nossa demonstração.

Deste teorema, temos $\operatorname{Alt}^{n}(V) \cong \mathbb{R}$ se $n=\operatorname{dim} V$ e, como já vimos antes, $\operatorname{Alt}^{p}(V)=0$ se $p>n$. No caso $n=\operatorname{dim} V$ temos que $\operatorname{dim} \operatorname{Alt}^{n}(V)=1$ e a base é dada pela $n$-forma alternada $\epsilon_{1} \wedge \ldots \wedge \epsilon_{n}$. Em particular toda $n$-forma alternada do $\mathbb{R}^{n}$ é proporcional ao determinando 0.1 .3 desse capítulo. 


\section{Capítulo}

\section{A cohomologia de de Rham}

\subsection{Formas diferenciais}

O objetivo deste capítulo é apresentar a cohomologia de de Rham e provar o lema de Poincaré.

Definição 1.1.1. Uma p-forma diferencial (ou simplesmente uma p-forma) é uma aplicação $\omega: U \longrightarrow A t^{p}\left(\mathbb{R}^{n}\right)$, onde $U$ é um aberto de $\mathbb{R}^{n}$ e $\omega$ é infinitamente diferenciável.

O conjunto de todas as $p$-formas diferenciáveis no aberto $U$ será denotado por $\Omega^{p}(U)$.

Denotaremos a derivada usual de $\omega$ por $\mathrm{D} \omega$, e seu valor em $x$ por $\mathrm{D}_{x} \omega$. Assim,

$$
\mathrm{D}_{x} \omega: \mathbb{R}^{n} \longrightarrow \operatorname{Alt}^{p}\left(\mathbb{R}^{n}\right)
$$

é tal que

$$
\left(\mathrm{D}_{x} \omega\right)\left(e_{i}\right)=\frac{d}{d t} \omega\left(x+t e_{i}\right)_{t=0}=\frac{\partial \omega}{\partial x_{i}}(x) .
$$

No capítulo anterior demonstramos que as $p$-formas $\epsilon_{I}=\epsilon_{i_{1}} \wedge \cdots \wedge \epsilon_{i_{p}}$ com $1 \leqslant i_{1}<$ $i_{2}<\cdots<i_{p} \leqslant n$ formam uma base para $\operatorname{Alt}^{p}\left(\mathbb{R}^{n}\right)$. Logo, qualquer $p$-forma $\omega \in \Omega^{p}(U)$ se escreve unicamente como:

$$
\omega(x)=\sum_{I} \omega_{I}(x) \epsilon_{I}
$$

onde $\omega_{I}(x)$ são funções reais diferenciáveis e $x \in U$. Lembramos ainda que $\operatorname{Alt}^{0}\left(\mathbb{R}^{n}\right)=\mathbb{R}$ e $\Omega^{0}(U)$ é o espaço das funções reais diferenciáveis em $U$, ou seja, $\Omega^{0}(U)=C^{\infty}(U, \mathbb{R})$. 
Assim, a diferencial $\mathrm{D}_{x} \omega$ é a aplicação linear dada por:

$$
\left(\mathrm{D}_{x} \omega\right)\left(e_{j}\right)=\sum_{j} \frac{\partial \omega_{I}}{\partial x_{j}}(x) \epsilon_{I}, j=1, \cdots, n .
$$

E note que a função $x \longrightarrow \mathrm{D}_{x} \omega$ é uma aplicação de $U$ no espaço das aplicações lineares de $\mathbb{R}^{n}$ em $\operatorname{Alt}^{p}\left(\mathbb{R}^{n}\right)$.

Definição 1.1.2. A diferencial exterior é o operador linear

$$
d: \Omega^{p}(U) \longrightarrow \Omega^{p+1}(U)
$$

tal que

$$
d_{x} \omega\left(\xi_{1}, \ldots, \xi_{p+1}\right)=\sum_{1}^{p+1}(-1)^{l-1} D_{x} \omega\left(\xi_{l}\right)\left(\xi_{1}, \ldots, \widehat{\xi}_{l}, \ldots, \xi_{p+1}\right)
$$

onde $\left(\xi_{1}, \ldots, \widehat{\xi}_{l}, \ldots, \xi_{p+1}\right)=\left(\xi_{1}, \ldots, \xi_{l-1}, \xi_{l+1}, \ldots, \xi_{p+1}\right)$.

Mostremos que $d_{x} \omega \in \operatorname{Alt}^{p+1}\left(\mathbb{R}^{n}\right)$. Pelo lema 0.2 .7 do capítulo anterior, basta mostrar que se $\xi_{i}=\xi_{i+1}$, então $d_{x} \omega\left(\xi_{1}, \ldots, \xi_{p+1}\right)=0$, para qualquer $1 \leqslant i \leqslant p-1$. Assim:

$$
\begin{aligned}
& \sum_{1}^{p+1}(-1)^{l-1} \mathrm{D}_{x} \omega\left(\xi_{l}\right)\left(\xi_{1}, \ldots, \widehat{\xi}_{l}, \ldots, \xi_{p+1}\right)= \\
& =(-1)^{i-1} \mathrm{D}_{x} \omega\left(\xi_{i}\right)\left(\xi_{1}, \ldots, \widehat{\xi}_{i}, \ldots, \xi_{p+1}\right)+(-1)^{i} \mathrm{D}_{x} \omega\left(\xi_{i+1}\right)\left(\xi_{1}, \ldots, \widehat{\xi_{i+1}}, \ldots, \xi_{p+1}\right)= \\
& =(-1)^{i-1} \mathrm{D}_{x} \omega\left(\xi_{i}\right)\left(\xi_{1}, \ldots, \widehat{\xi}_{i}, \ldots, \xi_{p+1}\right)+(-1)^{i} \mathrm{D}_{x} \omega\left(\xi_{i}\right)\left(\xi_{1}, \ldots, \widehat{\xi}_{i}, \ldots, \xi_{p+1}\right)=0
\end{aligned}
$$

Exemplo 1.1.3. Tomemos a i-ésima projeção $x_{i}: U \longrightarrow \mathbb{R}$. Assim:

$$
D_{x} x_{i}\left(e_{j}\right)=\sum_{j=1}^{n} \frac{\partial x_{i}}{\partial x_{j}}(x) \epsilon_{j}=\epsilon_{i}
$$

pois $\frac{\partial x_{i}}{\partial x_{j}}= \begin{cases}0 & \text { se }, i \neq j \\ 1 & \text { se, } i=j .\end{cases}$

De uma forma mais geral, se $f \in \Omega^{0}(U)$, teremos:

$$
d_{x} f(\zeta)=\frac{\partial f}{\partial x_{1}}(x) \zeta^{1}+\frac{\partial f}{\partial x_{2}}(x) \zeta^{2}+\cdots+\frac{\partial f}{\partial x_{n}}(x) \zeta^{n}
$$

$\operatorname{com} \zeta=\left(\zeta_{1}, \ldots, \zeta_{n}\right)$, ou seja, 


$$
d f=\sum_{i=1}^{n} \frac{\partial f}{\partial x_{i}} \epsilon^{i}=\sum_{i=1}^{n} \frac{\partial f}{\partial x_{i}} d x_{i}
$$

Lema 1.1.4. Se $\omega(x)=f(x) \epsilon_{I}$, então $d_{x} \omega=d_{x} f \wedge \epsilon_{I}$.

Demonstração: Pela equação (\$), temos:

$$
\mathrm{D}_{x} \omega(\zeta)=\mathrm{D}_{x} f(\zeta) \epsilon_{I}=\left(\frac{\partial f}{\partial x_{1}} \zeta^{1}+\frac{\partial f}{\partial x_{2}} \zeta^{2}+\cdots+\frac{\partial f}{\partial x_{n}} \zeta^{n}\right) \epsilon_{I}=d_{x} f(\zeta) \epsilon_{I}
$$

e, usando a definição de diferencial exterior, temos:

$$
\begin{aligned}
d_{x} \omega\left(\xi_{1}, \ldots, \xi_{p+1}\right) & =\sum_{1}^{p+1}(-1)^{l+1} \mathrm{D}_{x} \omega\left(\xi_{l}\right)\left(\xi_{1}, \ldots, \widehat{\xi}_{l}, \ldots, \xi_{p+1}\right) \\
& =\sum_{1}^{p+1}(-1)^{l+1} d_{x} f\left(\xi_{l}\right) \epsilon_{I}\left(\xi_{1}, \ldots, \widehat{\xi}_{l}, \ldots, \xi_{p+1}\right) .
\end{aligned}
$$

Notemos que esta última expressão é similar a definição de produto exterior dada no capítulo anterior tomando $\omega_{1}=d_{x} f$ e $\omega_{2}=\epsilon_{I}$. Além disso, o somatório acima percorre todas $p+1$ embaralhamentos do tipo $(1, p)$ existentes, e o fator $(-1)^{p+1}$ é o sinal da permutação correspondente.

Notemos que

$$
\epsilon^{k} \wedge \epsilon^{I}=\left\{\begin{array}{cc}
0 & \text { se, } k \in I \\
(-1)^{r} \epsilon_{J} & \text { se, } k \notin I
\end{array}\right.
$$

onde $\epsilon_{I} \in \operatorname{Alt}^{p}\left(\mathbb{R}^{n}\right)$ e $r$ é o número determinado por $i_{r}<k<i_{r+1}$ e $J=\left(i_{1}, \ldots, i_{r}, k, i_{r+1}, \ldots, i_{p}\right)$.

Lema 1.1.5. Se $p \geqslant 0$, a composição $\Omega^{p}(U) \stackrel{d}{\longrightarrow} \Omega^{p+1} \stackrel{d}{\longrightarrow} \Omega^{p+2}$ é identicamente nula.

Demonstração: Lembrando que as aplicações $\epsilon_{I}$ formam uma base para $\operatorname{Alt}^{p}\left(\mathbb{R}^{n}\right)$, tomemos $\omega=f \epsilon_{I}$. Então:

$$
d \omega=d f \wedge \epsilon_{I}=\frac{\partial f}{\partial x_{1}} \epsilon_{1} \wedge \epsilon_{I}+\frac{\partial f}{\partial x_{2}} \epsilon_{2} \wedge \epsilon_{I}+\cdots+\frac{\partial f}{\partial x_{n}} \epsilon_{n} \wedge \epsilon_{I}=\sum_{i=1}^{n} \frac{\partial f}{\partial x_{i}} \epsilon_{i} \wedge \epsilon_{I} .
$$

Como $\epsilon_{i} \wedge \epsilon_{i}=0$ e $\epsilon_{i} \wedge \epsilon_{j}=-\epsilon_{j} \wedge \epsilon_{i}$, teremos: 


$$
\begin{aligned}
d^{2} \omega & =d\left(d f \wedge \epsilon_{I}\right) \\
& =d\left(\sum_{i=1}^{n} \frac{\partial f}{\partial x_{i}} \epsilon_{i} \wedge \epsilon_{I}\right) \\
& =\sum_{j=1}^{n} \sum_{i=1}^{n} \frac{\partial^{2} f}{\partial x_{i} \partial x_{j}} \epsilon_{i} \wedge \epsilon_{j} \wedge \epsilon_{I} \\
& =\sum_{i, j=1}^{n} \frac{\partial^{2} f}{\partial x_{i} \partial x_{j}} \epsilon_{i} \wedge \epsilon_{j} \wedge \epsilon_{I} \\
& =\sum_{i<j}^{n}\left(\frac{\partial^{2} f}{\partial x_{i} \partial x_{j}}-\frac{\partial^{2} f}{\partial x_{j} \partial x_{i}}\right) \epsilon_{i} \wedge \epsilon_{j} \wedge \epsilon_{I}=0
\end{aligned}
$$

O produto exterior em $\operatorname{Alt}^{*}\left(\mathbb{R}^{n}\right)$ induz um produto exterior em $\Omega^{*}(U)$, definido por:

$$
\begin{aligned}
& \wedge: \Omega^{p}(U) \times \Omega^{q}(U) \quad \longrightarrow \quad \Omega^{p+q}(U) \\
& \left(\omega_{1} \wedge \omega_{2}\right)(x) \longmapsto \omega_{1}(x) \wedge \omega_{2}(x)
\end{aligned}
$$

Ou seja, se $\omega_{1}$ é uma $p$-forma diferencial e $\omega_{2}$ é uma $q$-forma diferencial, teremos que $\omega_{1} \wedge \omega_{2}$ será uma $p+q$-forma diferencial e a operação $\wedge$ será bilinear. Mais ainda, se $f \in C^{\infty}(U, \mathbb{R})$, temos:

$$
\left(f \omega_{1}\right) \wedge \omega_{2}=f\left(\omega_{1} \wedge \omega_{2}\right)=\omega_{1} \wedge\left(f \omega_{2}\right)
$$

isso segue do fato de que $f \in \Omega^{0}(U)$, sendo assim, $f \wedge \omega=f \omega$, pela definição de produto exterior.

Lema 1.1.6. Para $\omega_{1} \in \Omega^{p}(U)$ e $\omega_{2} \in \Omega^{q}(U)$, temos

$$
d\left(\omega_{1} \wedge \omega_{2}\right)=d \omega_{1} \wedge \omega_{2}+(-1)^{p} \omega_{1} \wedge d \omega_{2}
$$

Demonstração: Sendo as formas $\epsilon_{I}$ base, é suficiente provar para o caso $\omega_{1}=f \epsilon_{I}$ e $\omega_{2}=g \epsilon_{J}$. Assim $\omega_{1} \wedge \omega_{2}=f \epsilon_{I} \wedge g \epsilon_{J}=f g \epsilon_{I} \wedge \epsilon_{J}$, pois $f, g \in \Omega^{0}(U)$. Logo:

$$
\begin{aligned}
d\left(\omega_{1} \wedge \omega_{2}\right) & =d\left(f g \epsilon_{I} \wedge \epsilon_{J}\right) \\
& =d f g \wedge \epsilon_{I} \wedge \epsilon_{J}+f d g \wedge \epsilon_{I} \wedge \epsilon_{J} \\
& =d f \wedge \epsilon_{I} \wedge g \epsilon_{J}+f d g \wedge \epsilon_{I} \wedge \epsilon_{J} \\
& =d f \wedge \epsilon_{I} \wedge g \epsilon_{J}+(-1)^{p} f \epsilon_{I} \wedge d g \wedge \epsilon_{J} \\
& =d \omega_{1} \wedge \omega_{2}+(-1)^{p} \omega_{1} \wedge d \omega_{2}
\end{aligned}
$$

onde usamos o lema 0.2 .8 na última passagem com o fato de que $d g \in \Omega^{1}(U)$ e $\epsilon_{I} \in \Omega^{p}(U)$ 
Resumindo, temos uma álgebra anti-comutativa $\Omega^{*}(U)$, com uma diferencial $d: \Omega^{*}(U) \longrightarrow$ $\Omega^{*+1}(U)$ e $d \circ d=0$. A cadeia:

$$
\Omega^{0}(U) \stackrel{d}{\longrightarrow} \Omega^{1}(U) \longrightarrow \cdots \longrightarrow \Omega^{p}(U) \stackrel{d}{\longrightarrow} \Omega^{p+1}(U) \longrightarrow \cdots
$$

é chamada de complexo de de Rham de $U$.

Teorema 1.1.7. Há precisamente um operador linear

$$
d: \Omega^{p}(U) \longrightarrow \Omega^{p+1}(U) \quad, \quad p=0,1, \ldots
$$

tal que :

(i) $f \in \Omega^{0}(U), d f=\frac{\partial f}{\partial x_{1}} \epsilon_{1}+\cdots+\frac{\partial f}{\partial x_{n}} \epsilon_{n}$;

(ii) $d \circ d=0$;

(iii) $d\left(\omega_{1} \wedge \omega_{2}\right)=d \omega_{1} \wedge \omega_{2}+(-1)^{p} \omega_{1} \wedge d \omega_{2}$.

Demonstração: Ora, nos três lemas anteriores provamos que a diferencial $d$ satisfaz as três condições impostas em (i), (ii) e (iii) no enunciado. Resta-nos provar a unicidade. Suponhamos que exista um outro operador $d^{\prime}$ que satisfaz as condições (i), (ii) e (iii) do enunciado. Por (i) teremos que $d^{\prime}=d$ em $\Omega^{0}(U)$. Em particular, para a $i$-ésima projeção $x_{i}: U \longrightarrow \mathbb{R}$, teremos $d x_{i}=d^{\prime} x_{i}$. Seguindo o mesmo raciocínio do exemplo $1.1 .3, d^{\prime} x_{i}=\epsilon_{i}$, função constante. Pela propriedade (ii), temos $d^{\prime} \circ d^{\prime}=0$, então $d^{\prime}\left(d^{\prime} x_{i}\right)=d^{\prime} \epsilon^{i}=0$, e por (iii), $d^{\prime} \epsilon_{I}=0$.

Tomemos $\omega=f \epsilon_{I}=f \wedge \epsilon_{I}, \operatorname{com} f \in C^{\infty}(U, \mathbb{R})$, então:

$$
d^{\prime} \omega=d^{\prime}\left(f \wedge \epsilon_{I}\right)=d^{\prime} f \wedge \epsilon_{I}+f \wedge d^{\prime} \epsilon_{I}=d^{\prime} f \wedge \epsilon_{I}=d f \wedge \epsilon_{I}=d \omega
$$

Logo, $d=d^{\prime}$, para todo $\omega \in \Omega^{p}(U)$.

Vejamos dois exemplos de cálculo da diferencial.

Exemplo 1.1.8. Seja $U \subset \mathbb{R}^{3}$ um aberto e $d: \Omega^{1}(U) \longrightarrow \Omega^{2}(U)$. 


$$
\begin{aligned}
d\left(f_{1} \epsilon_{1}+f_{2} \epsilon_{2}+f_{3} \epsilon_{3}\right) & =d f_{1} \wedge \epsilon_{1}+d f_{2} \wedge \epsilon_{2}+d f_{3} \wedge \epsilon_{3}+ \\
& =\left(\frac{\partial f_{1}}{\partial x_{1}} \epsilon_{1} \wedge \epsilon_{1}+\frac{\partial f_{1}}{\partial x_{2}} \epsilon_{2} \wedge \epsilon_{1}+\frac{\partial f_{1}}{\partial x_{3}} \epsilon_{3} \wedge \epsilon_{1}\right)+ \\
& +\left(\frac{\partial f_{2}}{\partial x_{1}} \epsilon_{1} \wedge \epsilon_{2}+\frac{\partial f_{2}}{\partial x_{2}} \epsilon_{2} \wedge \epsilon_{2}+\frac{\partial f_{2}}{\partial x_{3}} \epsilon_{3} \wedge \epsilon_{2}\right)+ \\
& +\left(\frac{\partial f_{3}}{\partial x_{1}} \epsilon_{1} \wedge \epsilon_{3}+\frac{\partial f_{3}}{\partial x_{2}} \epsilon_{2} \wedge \epsilon_{3}+\frac{\partial f_{3}}{\partial x_{3}} \epsilon_{3} \wedge \epsilon_{3}\right) \\
& =\left(\frac{\partial f_{2}}{\partial x_{1}}-\frac{\partial f_{1}}{\partial x_{2}}\right) \epsilon_{1} \wedge \epsilon_{2}+\left(\frac{\partial f_{2}}{\partial x_{3}}-\frac{\partial f_{3}}{\partial x_{2}}\right) \epsilon_{3} \wedge \epsilon_{2}+ \\
& +\left(\frac{\partial f_{1}}{\partial x_{3}}-\frac{\partial f_{3}}{\partial x_{1}}\right) \epsilon_{3} \wedge \epsilon_{1}
\end{aligned}
$$

onde usamos na primeira igualdade o teorema anterior no fato de que $d\left(f \wedge \epsilon_{i}\right)=d f_{i} \wedge$ $\epsilon_{i}+(-1)^{1} f_{i} \wedge d \epsilon_{i}=d f_{i} \wedge \epsilon_{i}$, e na última igualdade usamos que $\epsilon_{i} \wedge \epsilon_{j}=-\epsilon_{j} \wedge \epsilon_{i}$, pois ambas são 1-formas.

Exemplo 1.1.9. Seja $U \subset \mathbb{R}^{3}$ um aberto e $d: \Omega^{1}(U) \longrightarrow \Omega^{2}(U)$.

$$
\begin{aligned}
d\left(g_{3} \epsilon_{1} \wedge \epsilon_{2}+g_{1} \epsilon_{2} \wedge \epsilon_{3}+g_{2} \epsilon_{3} \wedge \epsilon_{1}\right) & =d g_{3} \wedge \epsilon_{1} \wedge \epsilon_{2}+d g_{1} \wedge \epsilon_{2} \wedge \epsilon_{3}+d g_{2} \wedge \epsilon_{3} \wedge \epsilon_{1} \\
& =\frac{\partial g_{3}}{\partial x_{1}} \epsilon_{1} \wedge \epsilon_{1} \wedge \epsilon_{2}+\frac{\partial g_{3}}{\partial x_{2}} \epsilon_{2} \wedge \epsilon_{1} \wedge \epsilon_{2}+\frac{\partial g_{3}}{\partial x_{3}} \epsilon_{3} \wedge \epsilon_{1} \wedge \epsilon_{2}+ \\
& +\frac{\partial g_{1}}{\partial x_{1}} \epsilon_{1} \wedge \epsilon_{2} \wedge \epsilon_{3}+\frac{\partial g_{1}}{\partial x_{2}} \epsilon_{2} \wedge \epsilon_{2} \wedge \epsilon_{3}+\frac{\partial g_{1}}{\partial x_{3}} \epsilon_{3} \wedge \epsilon_{2} \wedge \epsilon_{3}+ \\
& +\frac{\partial g_{2}}{\partial x_{1}} \epsilon_{1} \wedge \epsilon_{3} \wedge \epsilon_{1}+\frac{\partial g_{2}}{\partial x_{2}} \epsilon_{2} \wedge \epsilon_{3} \wedge \epsilon_{1}+\frac{\partial g_{2}}{\partial x_{3}} \epsilon_{1} \wedge \epsilon_{3} \wedge \epsilon_{1} \\
& =\left(\frac{\partial g_{1}}{\partial x_{1}}+\frac{\partial g_{2}}{\partial x_{2}}+\frac{\partial g_{3}}{\partial x_{3}}\right) \epsilon_{1} \wedge \epsilon_{2} \wedge \epsilon_{3} .
\end{aligned}
$$

\subsection{A cohomologia de de Rham}

Definição 1.2.1. A p-ésima cohomologia de de Rham é o espaço vetorial quociente:

$$
H^{p}(U)=\frac{\operatorname{ker}\left(d: \Omega^{p}(U) \longrightarrow \Omega^{p+1}(U)\right)}{i m\left(d: \Omega^{p-1}(U) \longrightarrow \Omega^{p}(U)\right)} .
$$


Em particular $H^{p}(U)=0$ para $p<0$ e $H^{0}(U)$ é o núcleo de

$$
d: C^{\infty}(U, \mathbb{R}) \longrightarrow \Omega^{1}(U)
$$

é o espaço das funções $f \in C^{\infty}(U, \mathbb{R})$ cujas derivadas se anulam em $U$, ou seja,

$$
H^{0}(U)=\left\{f: U \longrightarrow \mathbb{R}: \frac{\partial f}{\partial x_{i}}(x)=0, \forall x \in U, 1 \leqslant i \leqslant n\right\}
$$

Em um conjunto aberto $U$ podemos estabelecer uma relação de equivalência onde $q_{1} \sim q_{2}$ se existir uma curva contínua $\alpha:[a, b] \longrightarrow U$ tal que $\alpha(a)=q_{1}$ e $\alpha(b)=q_{2}$. Essas classes de equivalência particionam $U$ em abertos disjuntos, aos quais nomeamos componentes conexas de $U$. Uma componente conexa $W$ de $U$ será dita maximal se não for possível escrever $W$ como união de dois abertos disjuntos e não vazios, na topologia induzida pelo $\mathbb{R}^{n}$. Lembrando que qualquer aberto $U \subseteq \mathbb{R}^{n}$ terá sempre uma quantidade enumerável de componentes conexas, isto porque, para cada componente conexa, podemos escolher um ponto com coordenadas racionais para representá-la.

Lema 1.2.2. $H^{0}(U)$ é o espaço vetorial das aplicações $U \longrightarrow \mathbb{R}$ que são constantes em cada componente conexa de $U$.

Demonstração: Funções $f: U \longrightarrow \mathbb{R}$ que são localmente constantes fornecem uma partição de $U$ em abertos disjuntos $f^{-1}(c), c \in \mathbb{R}$, ou seja, cada $f^{-1}(c)$ será uma componente conexa de $U$, logo, $f$ será localmente constante quando for constante em cada componente conexa.

A $\operatorname{dim}_{\mathbb{R}}\left(H^{0}(U)\right)$ será a quantidade de componentes conexas de $U$. Já citamos que as componentes conexas de $U$ são enumeráveis, $\operatorname{logo} \operatorname{dim}_{\mathbb{R}}\left(H^{0}(U)\right)$ será um número inteiro não negativo ou $\infty$.

Quando $\omega \in \Omega^{p}(U)$ pertence ao núcleo de $d: \Omega^{p}(U) \longrightarrow \Omega^{p+1}(U)$, ou seja, $d \omega=0$, dizemos que $\omega$ é uma $p$-forma fechada. As aplicações que fazem parte de $d\left(\Omega^{p-1}\right) \subset \Omega^{p}$ são chamadas de $p$-formas exatas. Note que toda $p$-forma exata é uma $p$-forma fechada, mas o inverso não é verdade. Isto é justamente o que mede a $p$-ésima cohomologia de de Rham, o quanto as $p$-formas fechadas são exatas. No caso de $H^{p}(U)=0$, teremos que todas as $p$-formas fechadas serão exatas. As $p$-formas fechadas determinam as classes de cohomologia, da seguinte forma:

$$
[\omega]=\omega+d\left(\Omega^{p-1}\right) \subset \Omega^{p}, \omega \text { fechada. }
$$


Duas classes de cohomologia serão iguais quando diferirem por uma forma exata, ou seja, $[\omega]=\left[\omega^{\prime}\right]$ se $\omega-\omega^{\prime}$ é exata. Em geral o espaço das $p$-formas fechadas e o espaço das $p$-formas exatas tem dimensão infinita, o que torna curioso o fato de que a dimensão de $H^{p}(U)$ ser, usualmente, finita.

Podemos definir um produto entre classes da seguinte forma:

$$
\begin{aligned}
H^{p}(U) \times H^{q}(U) & \longrightarrow H^{p+q}(U) \\
\left(\left[\omega_{1}\right],\left[\omega_{2}\right]\right) & \longmapsto\left[\omega_{1} \wedge \omega_{2}\right]
\end{aligned}
$$

Vejamos que este produto está bem definido, pois:

$$
\begin{aligned}
\left(\omega_{1}+d \eta_{1}\right) \wedge\left(\omega_{2}+d \eta_{2}\right) & =\omega_{1} \wedge \omega_{2}+d \eta_{1} \wedge \omega_{2}+\omega_{1} \wedge d \eta_{2}+d \eta_{1} \wedge d \eta_{2} \\
& =\omega_{1} \wedge \omega_{2}+d\left(\eta_{1} \wedge \omega_{2}+(-1)^{p} \omega_{1} \wedge \eta_{2}+\eta_{1} \wedge d \eta_{2}\right)
\end{aligned}
$$

Para conseguir um funtor contravariante de $U$ em $H^{p}(U)$, tomemos uma aplicação linear infinitamente diferenciável $\phi_{1}: U_{1} \longrightarrow U_{2}$ entre abertos $U_{1} \subset \mathbb{R}^{n}$ e $U_{2} \subset \mathbb{R}^{m}$. Gostaríamos de definir:

$$
H^{p}(\phi): H^{p}\left(U_{2}\right) \longrightarrow H^{p}\left(U_{1}\right)
$$

tal que,

$$
\begin{aligned}
H^{p}\left(\phi_{1} \circ \phi_{2}\right) & =H^{p}\left(\phi_{1}\right) \circ H^{p}\left(\phi_{2}\right) \\
H^{p}(\mathrm{id}) & =\mathrm{id} .
\end{aligned}
$$

Mas antes disso temos:

Definição 1.2.3. Sejam dois abertos $U_{1} \subset \mathbb{R}^{n}$ e $U_{2} \subset \mathbb{R}^{m}$ e $\phi: U_{1} \longrightarrow U_{2}$ uma aplicação diferenciável. Definimos o pullback de $\phi$ quando $p>0$ como:

$$
\begin{aligned}
& \Omega^{p}(\phi): \Omega^{p}\left(U_{2}\right) \longrightarrow \Omega^{p}\left(U_{1}\right) \\
& \Omega^{p}(\phi)(\omega)_{x}\left(\xi_{1}, \ldots, \xi_{n}\right)=\omega_{\phi(x)}\left(D_{x} \phi\left(\xi_{1}\right), \ldots, D_{x} \phi\left(\xi_{p}\right)\right),
\end{aligned}
$$

e quando $p=0: \Omega^{p}(\phi)(\omega)_{x}=(\omega)_{\phi(x)}$.

É comum encontrarmos na literatura a notação $\phi^{*}$ para $\Omega^{p}(\phi)$, que torna a notação mais simples. Neste trabalho adotaremos a notação de $\phi^{*}$.

Tomemos $\phi: U_{1} \longrightarrow U_{2}$ e $\psi: U_{2} \longrightarrow U_{3}$, onde $U_{1} \subset \mathbb{R}^{n}, U_{2} \subset \mathbb{R}^{m}$ e $U_{3} \subset \mathbb{R}^{q}$. Usando a regra da cadeia temos $\mathrm{D}_{x}(\psi \circ \phi)=\mathrm{D}_{\phi(x)}(\psi) \circ \mathrm{D}_{x}(\phi)$, que na definição 1.2.3, nos dá:

$$
(\psi \circ \phi)^{*}=\psi^{*} \circ \phi^{*} \text { é claro que } \phi^{*}\left(\operatorname{id}_{U}\right)=i d_{\Omega^{p}(U)}
$$


Provemos a afirmação acima. Temos:

$$
\begin{gathered}
U_{1} \stackrel{\phi}{\longrightarrow} U_{2} \stackrel{\psi}{\longrightarrow} U_{3} \\
\Omega^{p}\left(U_{3}\right) \stackrel{\psi^{*}}{\longrightarrow} \Omega^{p}\left(U_{2}\right) \stackrel{\phi *}{\longrightarrow} \Omega^{p}\left(U_{1}\right)
\end{gathered}
$$

Seja $x \in U_{1}$, faremos $\phi(x)=y$ e $\psi(y)=\psi(\phi(x))=z$. Então, para $\omega \in \Omega^{p}\left(U_{3}\right)$, $\xi_{1}, \ldots, \xi_{p} \in \mathbb{R}^{n}$ e $x \in U_{1}$, temos:

$$
\begin{aligned}
(\psi \circ \phi)_{x}^{*}(\omega)\left(\xi_{1}, \ldots, \xi_{p}\right) & =\omega_{(\psi \circ \phi)(x)}\left(\mathrm{D}_{x}(\psi \circ \phi)\left(\xi_{1}\right), \ldots, \mathrm{D}_{x}(\psi \circ \phi)\left(\xi_{p}\right)\right) \\
& =\omega_{z}\left(\mathrm{D}_{y} \psi\left(\mathrm{D}_{x}\left(\xi_{1}\right)\right), \ldots, \mathrm{D}_{y} \psi\left(\mathrm{D}_{x}\left(\xi_{p}\right)\right)\right)
\end{aligned}
$$

onde usamos a regra da cadeira na última igualdade. Por outro lado:

$$
\begin{aligned}
\left(\psi^{*} \circ \phi^{*}\right)_{x}(\omega)\left(\xi_{1}, \ldots, \xi_{p}\right) & =\psi^{*}\left(\omega_{\phi(x)}\left(\mathrm{D}_{x} \phi\left(\xi_{1}\right), \ldots, \mathrm{D}_{x} \phi\left(\xi_{p}\right)\right)\right) \\
& =\psi^{*}\left(\omega_{y}\left(\mathrm{D}_{x} \phi\left(\xi_{1}\right), \ldots, \mathrm{D}_{x} \phi\left(\xi_{p}\right)\right)\right) \\
& =\omega_{\psi(y)}\left(\mathrm{D}_{y} \psi\left(\mathrm{D}_{x}\left(\xi_{1}\right)\right), \ldots, \mathrm{D}_{y} \psi\left(\mathrm{D}_{x}\left(\xi_{p}\right)\right)\right) \\
& =\omega_{z}\left(\mathrm{D}_{y} \psi\left(\mathrm{D}_{x}\left(\xi_{1}\right)\right), \ldots, \mathrm{D}_{y} \psi\left(\mathrm{D}_{x}\left(\xi_{p}\right)\right)\right)
\end{aligned}
$$

o que prova a veracidade da primeira igualdade. Para a segunda igualdade, temos:

$$
\begin{array}{cl}
\operatorname{id}_{U}: U \longrightarrow U & ; \quad \operatorname{id}_{U}(x)=x \quad ; U \subset \mathbb{R}^{n} \\
\operatorname{id}^{*}: \Omega^{p}(U) \longrightarrow \Omega^{p}(U) & ; \quad \operatorname{id}^{*} \omega=\omega .
\end{array}
$$

Assim, para $x \in U, \omega \in \Omega^{p}(U)$ e $\xi_{1}, \ldots, \xi_{p} \in \mathbb{R}^{n}$, temos:

$$
\operatorname{id}_{x}^{*} \omega\left(\xi_{1}, \ldots, \xi_{p}\right)=\omega_{\operatorname{id}(x)}\left(D_{x} \operatorname{id}\left(\xi_{1}\right), \ldots, D_{x} \operatorname{id}\left(\xi_{p}\right)\right)=\omega_{x}\left(\xi_{1}, \ldots, \xi_{p}\right)
$$

pois $\mathrm{D}_{x} \mathrm{id}\left(\xi_{i}\right)=\xi_{i}$ pelo teorema 1.1.7. Logo, as duas identidades estão provadas.

Exemplo 1.2.4. Para 1-forma constante $\epsilon_{i} \in \Omega^{1}(U)$, temos:

$$
\phi^{*}\left(\epsilon_{i}\right)=\sum_{i=1}^{n} \frac{\partial \phi_{i}}{\partial x_{k}} \epsilon_{k}=d \phi_{i}
$$

com $\phi_{i}$ a i-ésima função coordenada. Seja $\zeta=\left(\zeta^{1}, \ldots, \zeta^{n}\right) \in \mathbb{R}^{n}$, temos: 


$$
\begin{aligned}
\phi^{*}\left(\epsilon_{i}\right)(\zeta) & =\epsilon_{i}\left(D_{x} \phi(\zeta)\right) \\
& =\epsilon_{i}\left(\sum_{k=1}^{m} \sum_{l=1}^{n} \frac{\partial \phi_{k}}{\partial x_{l}} \zeta^{l} \epsilon_{k}\right) \\
& =\sum_{l=1}^{n} \frac{\partial \phi_{i}}{\partial x_{l}} \epsilon_{l}(\zeta)=d \phi_{i}(\zeta) .
\end{aligned}
$$

Teorema 1.2.5. Pela definição 1.2.3 de pull back, temos:

(i) $\phi^{*}(\omega \wedge \tau)=\phi^{*}(\omega) \wedge \phi^{*}(\tau)$;

(ii) $\phi^{*}(f)=f \circ \phi$ se $f \in \Omega^{0}\left(U_{2}\right)$;

(iii) $d \phi^{*}(\omega)=\phi^{*}(d \omega)$

Reciprocamente, se $\phi^{\prime}: \Omega^{*}\left(U_{2}\right) \longrightarrow \Omega^{*}\left(U_{1}\right)$ é um operador que satisfaz as três condições impostas, então $\phi^{\prime}=\phi^{*}$.

Demonstração: Para a primeira afirmação tomemos $x \in U_{1}, \xi_{1}, \ldots, \xi_{p+q} \in \mathbb{R}^{n}, \omega \in$ $\Omega^{p}\left(U_{2}\right), \tau \in \Omega^{q}\left(U_{2}\right)$ e consideremos $p, q>0$. Assim:

$$
\begin{aligned}
& \phi^{*}(\omega \wedge \tau)_{x}\left(\xi_{1}, \ldots, \xi_{p+q}\right)=(\omega \wedge \tau)_{\phi(x)}\left(\mathrm{D}_{x} \phi\left(\xi_{1}\right), \ldots, \mathrm{D}_{x} \phi\left(\xi_{p+q}\right)\right) \\
& =\sum_{I} \operatorname{sign}(\sigma) \omega_{\phi(x)}\left(\mathrm{D}_{x} \phi\left(\xi_{\sigma(1)}\right), \ldots, \mathrm{D}_{x} \phi\left(\xi_{\sigma(p)}\right)\right) \cdot \tau_{\phi(x)}\left(\mathrm{D}_{x} \phi\left(\xi_{\sigma(p+1)}\right), \ldots, \mathrm{D}_{x} \phi\left(\xi_{\sigma(p+q)}\right)\right) \\
& =\sum_{I} \operatorname{sign}(\sigma) \phi^{*} \omega_{x}\left(\xi_{\sigma(1)}, \ldots, \xi_{\sigma(p)}\right) \cdot \phi^{*} \tau_{x}\left(\xi_{\sigma(p+1)}, \ldots, \xi_{\sigma(p+q)}\right) \\
& =\left(\phi^{*}(\omega)_{x} \wedge \phi^{*}(\tau)_{x}\right)\left(\xi_{1}, \ldots, \xi_{p+q}\right)
\end{aligned}
$$

No caso em que $p=0$ ou $q=0$ a prova é similar.

A prova de (ii) está contida na definição de $\phi^{*}$ em grau zero.

Resta-nos provar a afirmação (iii). Primeiramente mostremos para o caso $f \in \Omega^{0}\left(U_{2}\right)$. Temos:

$$
d f=\sum_{k=1}^{m} \frac{\partial f}{\partial x_{k}} \epsilon_{k}=\sum_{k=1}^{m} \frac{\partial f}{\partial x_{k}} \wedge \epsilon_{k}
$$

onde usamos a definição de produto exterior, o item (i) do teorema 1.1 .7 e $\epsilon_{k} \in \Omega^{1}\left(U_{2}\right)$ 
ter valor constante. Então:

$$
\begin{aligned}
\phi^{*}(d f) & =\sum_{k=1}^{m} \phi^{*}\left(\frac{\partial f}{\partial x_{k}}\right) \wedge \phi^{*}\left(\epsilon_{k}\right) \\
& =\sum_{k=1}^{m} \frac{\partial f}{\partial x_{k}} \circ \phi \wedge \sum_{l=1}^{n} \frac{\partial \phi_{k}}{\partial x_{l}} \epsilon_{l} \\
& =\sum_{k=1}^{m} \sum_{l=1}^{n}\left(\frac{\partial f}{\partial x_{k}} \circ \phi\right)\left(\frac{\partial \phi_{k}}{\partial x_{l}}\right) \epsilon_{l} \\
& =\sum_{l=1}^{n} \sum_{k=1}^{m}\left(\left(\frac{\partial f}{\partial x_{k}} \circ \phi\right) \frac{\partial \phi_{k}}{\partial x_{l}}\right) \epsilon_{l} \\
& =\sum_{l=1}^{n} \frac{\partial(f \circ \phi)}{\partial x_{l}} \epsilon_{l}=d(f \circ \phi)=d\left(\phi^{*}(f)\right)
\end{aligned}
$$

onde usamos (i) e (ii) já provados e a regra da cadeira na última igualdade.

Como em situações anteriores, é suficiente provar o caso geral para $\omega=f \epsilon_{I}=f \wedge \epsilon_{I}$, com $f \in \Omega^{0}\left(U_{2}\right)$. Pelo lema 1.1.6, temos $d \omega=d f \wedge \epsilon_{I}$, pois $d \epsilon_{I}=0$. Além disso,

$$
\begin{aligned}
d \phi^{*}\left(\epsilon_{I}\right) & =d\left(\phi^{*}\left(\epsilon_{i_{1}}\right) \wedge \cdots \wedge \phi^{*}\left(\epsilon_{i_{p}}\right)\right) \\
& =\sum(-1)^{k-1} \phi^{*}\left(\epsilon_{i_{1}}\right) \wedge \cdots \wedge d \phi^{*}\left(\epsilon_{i_{k}}\right) \wedge \cdots \phi^{*}\left(\epsilon_{i_{p}}\right)=0
\end{aligned}
$$

isso porque no exemplo anterior ao teorema mostramos que $\phi^{*} \epsilon_{i}=d \phi_{i}$ e assim $d \phi^{*} \epsilon_{i}=$ $d\left(d \phi_{i}\right)=0$.

Para que a notação fique mais clara, escreveremos

$$
d x_{I}=d x_{i_{1}} \wedge \cdots \wedge d x_{i_{p}}
$$

em vez de usar $\epsilon_{I}=\epsilon_{i_{1}} \wedge \cdots \wedge \epsilon_{i_{p}}$. Assim, uma $p$-forma arbitrária pode ser escrita como:

$$
\omega(x)=\sum_{I} \omega_{I}(x) d x_{I}
$$

onde $I$ percorre todas as sequência $1 \leqslant i_{1}<i_{2}<\ldots<i_{p} \leqslant n$. Do exemplo 1.2 .4 temos $\phi\left(d y_{i}\right)=d \phi_{i}$, onde $y_{i}: U_{2} \longrightarrow \mathbb{R}$ é a $i$-ésima função coordenada e $\phi_{i}=y_{i} \circ \phi$ a $i$-ésima coordenada de $\phi$.

Exemplo 1.2.6. Tomemos $\gamma:(a, b) \longrightarrow U$ uma curva suave em $U$, com $\gamma=\left(\gamma_{1}, \ldots, \gamma_{n}\right)$, 
$e \omega=f_{1} d x_{1}+\ldots+f_{n} d x_{n} \in \Omega^{1}(U)$. Assim:

$$
\begin{aligned}
\gamma^{*}(\omega) & =\gamma^{*}\left(f_{1} \wedge d x_{1}\right)+\cdots+\gamma^{*}\left(f_{n} \wedge d x_{n}\right) \\
& =\gamma^{*}\left(f_{1}\right) \wedge \gamma^{*}\left(d x_{1}\right)+\cdots+\gamma^{*}\left(f_{n}\right) \wedge \gamma^{*}\left(d x_{n}\right) \\
& =\left(f_{1} \circ \gamma\right) d \gamma_{1}+\cdots+\left(f_{n} \circ \gamma\right) d \gamma_{n} \\
& =\left[\left(f_{1} \circ \gamma\right) \gamma_{1}^{\prime}+\cdots+\left(f_{n} \circ \gamma\right) \gamma_{n}^{\prime}\right] d t=<f(\gamma(t)), \gamma^{\prime}(t)>d t
\end{aligned}
$$

onde $<,>$ é o produto interno usual.

Exemplo 1.2.7. Se $\phi$ é uma função diferenciável entre dois abertos de $\mathbb{R}^{n}$, temos:

$$
\begin{aligned}
\phi^{*}\left(d x_{1} \wedge \cdots \wedge d x_{n}\right) & =\phi^{*}\left(d x_{1}\right) \wedge \cdots \wedge \phi^{*}\left(d x_{n}\right) \\
& =d \phi^{*}\left(x_{1}\right) \wedge \cdots \wedge d \phi^{*}\left(x_{n}\right) \\
& =d \phi_{1} \wedge \cdots \wedge d \phi_{n} \\
& =\operatorname{det}\left(D_{x} \phi\right) d x_{1} \wedge \cdots \wedge d x_{n}
\end{aligned}
$$

onde a última igualdade é consequência do lema 0.2.13 do capitulo anterior.

Exemplo 1.2.8. Tomemos

$$
\begin{aligned}
\phi: \mathbb{R}^{n} \times \mathbb{R} & \longrightarrow \mathbb{R}^{n} \\
(x, t) & \longmapsto \phi(x, t)=\psi(t) x
\end{aligned}
$$

onde $\psi \in C^{\infty}(\mathbb{R}, \mathbb{R})$. Então:

$$
\begin{aligned}
\phi^{*}\left(d x_{i}\right) & =d\left(\phi^{*} x_{i}\right) \\
& =d\left(\phi \circ x_{i}\right) \\
& =d\left(\psi(t) \cdot x_{i}\right) \\
& =x_{i} \psi^{\prime}(t) d t+\psi(t) d x_{i}
\end{aligned}
$$

Definição 1.2.9. Para uma aplicação diferenciável $\phi: U_{1} \longrightarrow U_{2}$, podemos associar a aplicação linear:

$$
\begin{aligned}
H^{p}(\phi): H^{p}\left(U_{2}\right) & \longrightarrow H^{p}\left(U_{1}\right) \\
{[\omega] } & \longmapsto\left[\phi^{*}(\omega)\right]
\end{aligned}
$$


Veja que a aplicação independe da escolha do representante, pois:

$$
\phi^{*}(\omega+d \eta)=\phi^{*}(\omega)+\phi^{*}(d \eta)=\phi^{*}(\omega)+d\left(\phi^{*} \eta\right) \in H^{p}\left(U_{1}\right)
$$

Além disso, se $\omega_{1} \in H^{p}\left(U_{2}\right)$ e $\omega_{2} \in H^{q}\left(U_{2}\right)$, temos:

$$
\begin{aligned}
H^{p+q}(\phi)\left(\left[\omega_{1}\right]\left[\omega_{2}\right]\right) & =H^{p+q}(\phi)\left(\left[\omega_{1} \wedge \omega_{2}\right]\right) \\
& =\left[\phi^{*}\left(\omega_{1} \wedge \omega_{2}\right)\right] \\
& =\left[\phi^{*} \omega_{1} \wedge \phi^{*} \omega_{2}\right] \\
& =\left[\phi^{*} \omega_{1}\right]\left[\phi^{*} \omega_{2}\right] \\
& =H^{p}(\phi)\left[\omega_{1}\right] \cdot H^{q}(\phi)\left[\omega_{2}\right]
\end{aligned}
$$

ou seja, $H^{p}(\phi): H^{p}\left(U_{2}\right) \longrightarrow H^{p}\left(U_{1}\right)$ é um homomorfismo de álgebras graduadas.

\subsection{O lema de Poincaré}

Lema 1.3.1. Se $U \subset \mathbb{R}^{n}$ é um aberto estrelado, existe um operador linear

$$
S_{p}: \Omega^{p}(U) \longrightarrow \Omega^{p-1}(U)
$$

tal que $d S_{p}+S_{p+1} d=i d$ quando $p>0$ e $S_{1} d=i d-e$, com $e(\omega)=\omega(0)$, para $\omega \in \Omega^{0}(U)$

Demonstração: Primeiramente lembremos que um conjunto estrelado é aquele que contém um ponto que pode ser conectado a outro ponto qualquer do conjunto por uma aplicação linear. No nosso caso podemos assumir $U$ como estrelado na origem, sem perda de generalidade, caso contário uma simples translação em $\psi$ resolveria o problema.

Consideremos o conjunto $\Omega^{p}(U \times \mathbb{R})$. Para cada $\omega \in \Omega^{p}(U, \mathbb{R})$, podemos escrever:

$$
\omega=\sum_{I} f_{I}(x, t) d x_{I}+\sum_{J} g_{J}(x, t) d t \wedge d x_{J}
$$

onde $I=\left(i_{1}, \ldots, i_{p}\right)$ e $J=\left(j_{1}, \ldots, j_{p-1}\right)$. Notemos que ao fazer isso separamos os termos que compõem $\omega$, sendo os primeiros aqueles que independem de $d t$ na combinação e o segundo somatório todos os termos que de $\omega$ que contém $d t$ na combinação. 
Definimos:

$$
\begin{aligned}
\widehat{S}_{p}: \Omega^{p}(U \times \mathbb{R}) & \longrightarrow \Omega^{p-1}(U) \\
\omega & \longmapsto \sum_{J}\left(\int_{1}^{0} g_{J}(x, t) d t\right) d x_{J} .
\end{aligned}
$$

Então:

$$
\begin{aligned}
d \widehat{S}_{p}(\omega) & =d\left(\sum_{J} \int_{1}^{0} g_{J}(x, t) d t\right) d x_{J} \\
& =\sum_{J, i}\left(\int_{0}^{1} \frac{\partial g_{J}(x, t)}{\partial x_{i}} d t\right) d x_{i} \wedge d x_{J}
\end{aligned}
$$

Temos também:

$$
\begin{aligned}
\widehat{S}_{p+1} d(\omega) & =\widehat{S}_{p+1}\left(\sum_{I} \frac{\partial f_{I}(x, t)}{\partial t} d t \wedge d x_{I}-\sum_{J, i} \frac{\partial g_{J}(x, t)}{\partial x_{i}} d t \wedge d x_{i} \wedge d x_{J}\right) \\
& =\sum_{I} \int_{0}^{1}\left(\frac{\partial f_{I}(x, t)}{\partial t} d t\right) d x_{I}-\sum_{J, i}\left(\int_{0}^{1} \frac{\partial g_{J}(x, t)}{\partial x_{i}} d t\right) d x_{i} \wedge d x_{J}
\end{aligned}
$$

E assim,

$$
\begin{aligned}
d \widehat{S}_{p}(\omega)+\widehat{S}_{p+1} d(\omega) & =\sum_{I} \int_{0}^{1}\left(\frac{\partial f_{I}(x, t)}{\partial t} d t\right) d x_{I} \\
& =\sum_{I} f_{I}(x, 1) d x_{I}-\sum_{I} f_{I}(x, 0) d x_{I}
\end{aligned}
$$

Até este ponto da demonstração, ainda não usamos a hipótese de que $U$ é um aberto estrelado. Usaremos no seguinte operador:

$$
\phi: U \times \mathbb{R} \longrightarrow U \quad, \quad \phi(x, t)=\psi(t) x
$$

onde $\phi$ é uma curva suave com:

$$
\left\{\begin{array}{ccc}
\psi(t)=0 & \text { se } & t \leqslant 0 \\
\psi(t)=1 & \text { se } & t \geqslant 1 \\
0 \leqslant \psi(t) \leqslant 1 & \text { se } & 0<t<1
\end{array}\right.
$$

como $U$ é estrelado, com as condições acima, $\phi(x) \in U$, qualquer que seja $x \in U$. Então, fica bem definida:

$$
\begin{aligned}
S_{p}: \Omega^{p}(U) & \longrightarrow \Omega^{p-1}(U) \\
\omega & \longmapsto \widehat{S}_{p}\left(\phi^{*}(\omega)\right) .
\end{aligned}
$$




$$
\begin{gathered}
\text { Tomando } \omega=\sum_{I} h_{I}(x) d x_{I} \text { e usando a diferencial como na definição } 1.2 .9 \\
\phi^{*}(\omega)=\sum h_{I}(\psi(t) x)\left(d \psi(t) x_{i_{1}}+\psi(t) d x_{i_{1}}\right) \wedge \cdots \wedge\left(d \psi(t) x_{i_{p}}+\psi(t) d x_{i_{p}}\right)
\end{gathered}
$$

onde, usando a notação na qual definimos $S_{p}$, teremos

$$
\sum_{I} f_{I}(x, t) d x_{I}=\sum_{I} h_{I}(\psi(t) x) \psi(t)^{p} d x_{I}
$$

o que implica

$$
\begin{aligned}
d S_{p}(\omega)+S_{p+1} d(\omega) & =\sum_{I} h_{I}(\psi(1) x) \psi(1)^{p} d x_{I}-\sum_{I} h_{I}(\psi(0) x) \psi(0)^{p} d x_{I} \\
& =\sum_{I} h_{I}(x) d x_{I}=\omega
\end{aligned}
$$

Para $p>0$, pois $\psi(0)=0$. No caso $p=0, \omega=h(x)$ e assim:

$$
d S_{0}(\omega)+S_{1} d(\omega)=h(x)-h(0)=\omega(x)=\omega(0) .
$$

E temos:

$$
d S_{p}(\omega)+S_{p+1} d(\omega)=\left\{\begin{array}{ccc}
\omega & \text { se } & p>0 \\
\omega(x)-\omega(0) & \text { se } & p=0
\end{array}\right.
$$

o que concluí a nossa demonstração.

Teorema 1.3.2. (Lema de Poincaré) Se U é um aberto estrelado, então, $H^{p}(U)=0$, para $p>0$ e $H^{0}(U)=\mathbb{R}$.

Ora, tomemos o operador $S_{p}$ do lema anterior. Para $\omega \in \Omega^{p}(U)$ uma $p$-forma fechada, com $p>0$, temos

$$
d S_{p}(\omega)+S_{p+1} d(\omega)=\omega \Rightarrow d S_{p}(\omega)=\omega
$$

pois $\omega$ é fechada e assim $S_{p+1} d(\omega)=0$. Logo, toda $p$-forma fechada é também exata. Assim $H^{p}(U)=0$, para $p>0$.

Para $p=0$, temos que $\omega(x)=\omega(0)$, ou seja, uma constante. Logo $H^{0}(U)=\mathbb{R}$. 


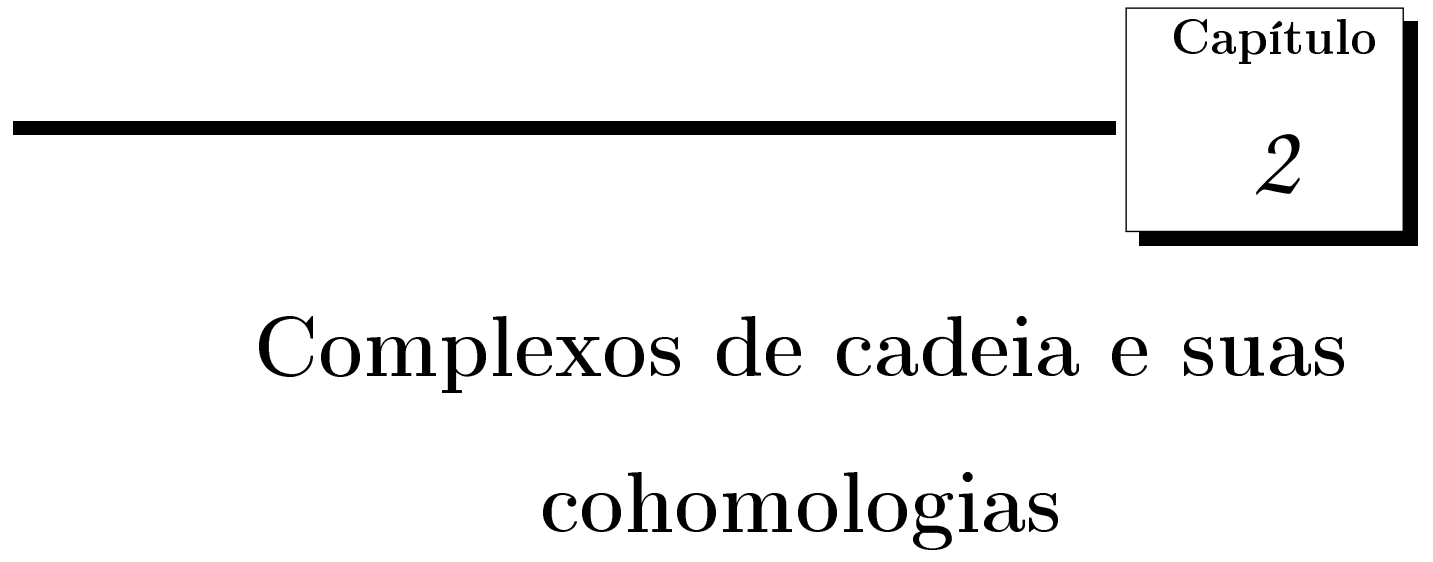

Neste capítulo apresentaremos algumas definições gerais de álgebra. Assim esclareceremos algumas construções do capítulo anterior e seremos capazes de construir alguns conceitos algébricos necessários para os próximos capítulos.

\subsection{Complexos de Cadeia}

Uma sequência de espaços vetoriais e aplicações lineares:

$$
A \stackrel{f}{\longrightarrow} B \stackrel{g}{\longrightarrow} C
$$

é chamada exata quando im $f=\operatorname{ker} g$ onde:

$$
\begin{aligned}
& \text { ker } g=\{b \in B \mid g(b)=0\}, \text { o núcleo de } g, \\
& \operatorname{im} f=\{f(a) \mid a \in A\}, \text { a imagem de } f .
\end{aligned}
$$

Note que $A \stackrel{f}{\longrightarrow} B \longrightarrow 0$ será exato quanto $f$ for sobrejetiva e que $0 \longrightarrow B \stackrel{g}{\longrightarrow} C$ 
será exato quando $g$ for injetiva. Uma sequência $A^{*}=\left\{A^{i}, d^{i}\right\}, i \in \mathbf{Z}$

$$
\cdots \longrightarrow A^{i-1} \stackrel{d^{i-1}}{\longrightarrow} A^{i} \stackrel{d^{i}}{\longrightarrow} A^{i+1} \stackrel{d^{i+1}}{\longrightarrow} A^{i+2} \longrightarrow \cdots
$$

de espaços vetoriais e aplicações lineares é chamada de complexo de cadeia quando $d^{i+1} \circ$ $d^{i}=0$, para qualquer $i \in \mathbf{Z}$, isto é im $d^{i} \subseteq \operatorname{ker} d^{i+1}$. Será exata quando ker $d^{i+1}=\operatorname{im} d^{i}$, para todo $i \in \mathbf{Z}$.

Uma sequência da forma:

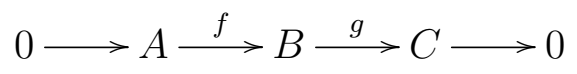

é chamada de exata curta. Isso significa que $f$ é injetiva, $g$ é sobrejetiva e im $f=\operatorname{ker} g$.

Toda sequencia exata longa como em (2), induzirá uma sequência exata curta, para cada $i \in \mathbf{Z}$,

$$
0 \longrightarrow \mathrm{im} d^{i-1} \longrightarrow A^{i} \longrightarrow \mathrm{im} d^{i} \longrightarrow 0
$$

que poderá ser usada para calcular $A^{i}$. Além disso os isomorfismos:

$$
\frac{A^{i-1}}{\operatorname{im} d^{i-2}} \cong \frac{A^{i-1}}{\operatorname{ker} d^{i-1}} \longrightarrow \operatorname{im} d^{i-1}
$$

são frequentemente aplicados em cálculos reais.

Lema 2.1.1. Suponha que $0 \longrightarrow A \stackrel{f}{\longrightarrow} B \stackrel{g}{\longrightarrow} C \longrightarrow 0$ é uma sequência exata curta de espaços vetoriais. Então $B$ tem dimensão finita se $A$ e $C$ tiverem. Além disso $B \cong$ $A \oplus C$.

Definição 2.1.2. Para um complexo de cadeia

$$
\cdots \longrightarrow A^{p-1} \stackrel{d^{p-1}}{\longrightarrow} A^{p} \stackrel{d^{p}}{\longrightarrow} A^{p+1} \stackrel{d^{p+1}}{\longrightarrow} A^{p+2} \longrightarrow \cdots
$$

definimos a p-ésima cohomologia por

$$
H^{p}\left(A^{*}\right)=\frac{\operatorname{ker} d^{p}}{\operatorname{im} d^{p-1}} .
$$


Os elementos de ker $d^{p}$ são chamados p-ciclos (ou fechados) e os elementos de im $d^{p-1}$ de p-fronteiras (ou exatos). Os elementos de $H^{p}\left(A^{*}\right)$ são chamados de classes de cohomologia.

Uma aplicação de cadeia $f: A^{*} \longrightarrow B^{*}$ entre complexos de cadeias consiste de aplicações lineares $f^{p}: A^{p} \longrightarrow B^{p}$ que satisfazem $d_{B}^{p} \circ f^{p}=f^{p+1} \circ d_{A}^{p}$. Uma aplicação de cadeia pode ser ilustrada através do seguinte diagrama comutativo:

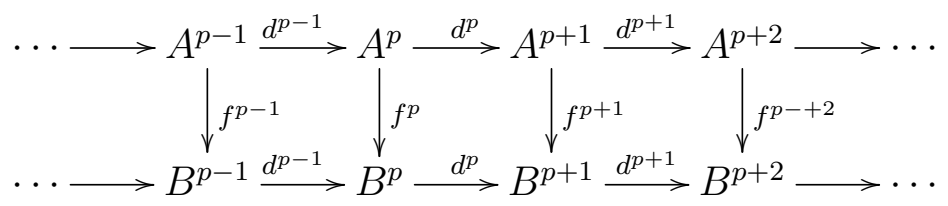

Lema 2.1.3. Uma aplicação de cadeia $f: A^{*} \longrightarrow B^{*}$ induz a seguinte aplicação linear:

$$
f^{*}=H^{*}(f): H^{p}\left(A^{*}\right) \longrightarrow H^{p}\left(B^{*}\right), \forall p
$$

Demonstração: Tomemos um ciclo $a \in A^{p},\left(d^{p}(a)=0\right)$ e sua classe de cohomologia:

$$
[a]=a+\operatorname{im} d^{p-1}
$$

Definimos

$$
f^{*}([a])=\left[f^{p}(a)\right]
$$

Primeiramente $f^{p}(a)$ é um ciclo pois

$$
d_{B}^{p}\left(f^{p}(a)\right)=f^{p+1}\left(d_{A}^{p}(a)\right)=f^{p+1}(0)=0
$$

Temos também que $\left[f^{p}(a)\right]$ independe da escolha do representante escolhido na classe $[a]$. Ora, se $\left[a_{1}\right]=\left[a_{2}\right]$, teremos que $a_{1}-a_{2} \in \operatorname{im~} d_{A}^{p-1}$, ou seja, $a_{1}-a_{2}=d_{A}^{p-1}(x)$, para algum $x \in A$. Logo:

$$
f^{p}\left(a_{1}\right)-f^{p}\left(a_{2}\right)=f^{p}\left(a_{1}-a_{2}\right)=f^{p} d_{A}^{p-1}(x)=d_{B}^{p-1} f^{p-1}(x)
$$

ou seja, $f^{p}\left(a_{1}\right)-f^{p}\left(a_{2}\right) \in \operatorname{im} d_{B}^{p-1}$ e, portanto, $f^{p}\left(a_{1}\right)$ e $f^{p}\left(a_{2}\right)$ definem a mesma classe de cohomologia.

Uma categoria $\mathcal{C}$ consiste de "objetos"e "morfismos" entre estes objetos, tal que a "composição" está definida, ou seja, se $f: C_{1} \longrightarrow C_{2}$ e $g: C_{2} \longrightarrow C_{3}$ são morfismos, então existe o morfismo $g \circ f: C_{1} \longrightarrow C_{3}$. Além disso devemos assumir que $\operatorname{id}_{C}: C \longrightarrow C$ é um morfismo para cada $C \in \mathcal{C}$.

O conceito ficara mais claro com alguns exemplos: 
Exemplo 2.1.4. A categoria de abertos em espaços euclidianos, onde os morfismos serão aplicações diferenciáveis.

Exemplo 2.1.5. A categoria de espaços vetoriais, onde os morfismos são aplicações lineares.

Exemplo 2.1.6. A categoria dos grupos abelianos, onde os morfismos são homomorfismos.

Exemplo 2.1.7. A categoria dos complexos de cadeias, onde os morfismos são as aplicações de cadeia.

Uma categoria com apenas um objeto é o mesmo que um semigrupo, ou seja, o semigrupo de morfismos do objeto.

Um funtor contravariante $F: \mathcal{C} \longrightarrow \mathcal{V}$ entre duas categorias é uma aplicação que associa a cada objeto $C \in \mathcal{C}$ a um objeto $F(C) \in \mathcal{V}$, e a cada morfismo $f: C_{1} \longrightarrow C_{2}$ em $\mathcal{C}$ a um morfismo $F(f): F\left(C_{2}\right) \longrightarrow F\left(C_{1}\right)$ em $\mathcal{V}$, tal que:

$$
F(g \circ f)=F(f) \circ F(g) \text { e } F\left(\operatorname{id}_{C}\right)=\operatorname{id}_{F(C)} .
$$

Um funtor covariante $F: \mathcal{C} \longrightarrow \mathcal{V}$ é uma aplicação $F(f): F\left(C_{1}\right) \longrightarrow F\left(C_{2}\right)$ tal que:

$$
F(g \circ f)=F(g) \circ F(f) \text { e } F\left(\operatorname{id}_{C}\right)=\operatorname{id}_{F(C)} .
$$

Logo, os funtores são aplicações que preservam as estruturas das categorias. Os contravariantes mudam a direção das aplicações e os covariantes as preservam. Vejamos alguns exemplos:

Exemplo 2.1.8. Seja $A$ um espaço vetorial e $F(C)=\operatorname{Hom}(C, A)$ as aplicações lineares de $C$ para $A$. Se $\phi: C_{1} \longrightarrow C_{2}$, então $\operatorname{Hom}(\phi, A): \operatorname{Hom}\left(C_{2}, A\right) \longrightarrow \operatorname{Hom}\left(C_{1}, A\right)$ é tal que $\psi \longmapsto \psi \circ \phi$ é um funtor contravariante da categoria de espaços vetoriais nela própria.

Do mesmo modo, $F(C)=\operatorname{Hom}(A, C) \operatorname{com} F(\phi): \psi \longmapsto \phi \circ \psi$ é um funtor covariante entre a categoria dos espaços vetoriais.

Exemplo 2.1.9. Tomemos a categoria $\mathcal{U}$ dos abertos em espaços euclidianos e aplicações diferenciáveis e Vect a categoria dos espaços vetoriais. O espaço vetorial $\Omega^{p}(U)$ das p- 
formas em $U \in \mathcal{U}$ define um funtor contravariante

$$
\Omega^{p}: \mathcal{U} \longrightarrow \mathcal{V e c t}
$$

Exemplo 2.1.10. Tomemos Vect* a categoria dos complexos de cadeia de espaços vetoriais. O complexo de de Rham define um funtor contravariante:

$$
\Omega^{*}: \mathcal{U} \longrightarrow \mathcal{V} e c t^{*}
$$

Exemplo 2.1.11. Cada p-ésima cohomologia $H^{p}: \mathcal{V} e c t^{*} \longrightarrow$ Vect é um funtor covariante.

Exemplo 2.1.12. A composição dos dois funtores acima é exatamente a cohomologia de de Rham, que é contravariante.

Uma sequência exata curta de complexos de cadeia

$$
0 \longrightarrow A^{*} \stackrel{f}{\longrightarrow} B^{*} \stackrel{g}{\longrightarrow} C^{*} \longrightarrow 0
$$

consiste de aplicações de cadeia $f$ e $g$ tal que

$$
0 \longrightarrow A^{p} \stackrel{f}{\longrightarrow} B^{p} \stackrel{g}{\longrightarrow} C^{p} \longrightarrow 0
$$

é uma sequência exata curta para todo $p$.

\subsection{Cohomologia de complexos de cadeia}

Lema 2.2.1. Para uma sequência exata curta de complexos de cadeias

$$
0 \longrightarrow A^{*} \stackrel{f}{\longrightarrow} B^{*} \stackrel{g}{\longrightarrow} C^{*} \longrightarrow 0
$$

teremos que a sequência

$$
H^{p}\left(A^{*}\right) \stackrel{f^{*}}{\longrightarrow} H^{p}\left(B^{*}\right) \stackrel{g^{*}}{\longrightarrow} H^{p}\left(C^{*}\right)
$$


é exata.

Demonstração: Como $0 \longrightarrow A^{p} \stackrel{f^{p}}{\longrightarrow} B^{p} \stackrel{g^{p}}{\longrightarrow} C^{p} \longrightarrow 0$ é exata para todo $p$, temos que $g^{p} \circ f^{p}=0$. Assim:

$$
g^{*} \circ f^{*}([a])=g^{*}\left(\left[f^{p}(a)\right)\right]=\left[g^{p}\left(f^{p}(a)\right)\right]=0
$$

para qualquer classe de cohomologia $[a] \in H^{p}(A)$, ou seja, im $f^{*} \subset \operatorname{ker} g^{*}$.

Reciprocamente, tomando $b \in H^{p}(B)$ tal que $g^{*}[b]=0$, temos:

$$
g^{p}(b)=d_{C}^{p-1}(c)
$$

Como $g^{p-1}$ é sobrejetiva, então existe $b_{1} \in B^{p-1} \operatorname{com} g^{p-1}(b)=c$, logo:

$$
\begin{aligned}
g^{p}\left(b-d_{B}^{p-1}\left(b_{1}\right)\right) & =g^{p}(b)-g\left(d_{B}^{p-1}\left(b_{1}\right)\right) \\
& =g^{p}(b)-d_{C}^{p-1}\left(g^{p-1}\left(b_{1}\right)\right) \\
& =g^{p}(b)-d_{C}^{p-1}(c)=0
\end{aligned}
$$

ou seja, $b-d_{B}^{p-1}\left(b_{1}\right) \in \operatorname{ker} g^{p}$. Como $\operatorname{ker} g=\operatorname{im} f$, existe $a \in H^{p}(A)$ tal que $f^{p}(a)=$ $b-d_{B}^{p-1}\left(b_{1}\right)$. Para mostrar que a é um $p$-ciclo é suficiente mostrar que $f^{p+1}\left(d_{A}^{p}(a)\right)=0$, pois $f$ é injetora,

$$
f^{p+1}\left(d_{A}^{p}(a)\right)=d_{B}^{p}\left(f^{p}(a)\right)=d_{B}^{p}\left(b-d_{B}^{p-1}\left(b_{1}\right)\right)=0
$$

pois $b$ é um $p$-ciclo e $d^{p} \circ d^{p-1}=0$. Encontramos assim uma classe de cohomologia $[a] \in H^{p}(A)$ tal que $f^{*}[a]=\left[b-d_{B}^{p-1}\left(b_{1}\right)\right]=[b]$, logo ker $g^{*} \subset \operatorname{im} f^{*}$, o que finaliza a nossa demonstração.

Definição 2.2.2. Para uma sequência exata curta de complexo de cadeia

$$
0 \longrightarrow A^{*} \stackrel{f}{\longrightarrow} B^{*} \stackrel{g}{\longrightarrow} C^{*} \longrightarrow 0
$$

definimos $\partial^{*}$ como a aplicação linear:

$$
\begin{aligned}
\partial^{*}: H^{p}\left(C^{*}\right) & \longrightarrow H^{p+1}\left(A^{*}\right) \\
{[c] } & \longmapsto\left[\left(f^{p+1}\right)^{-1}\left(d_{B}^{p}\left(\left(g^{p}\right)^{-1}(c)\right)\right)\right]
\end{aligned}
$$


A definição acima diz que para cada $b \in\left(g^{p}\right)^{-1}(c)$, temos $d_{B}^{p}(b) \in \operatorname{im} f^{p+1}$, e determina unicamente $a \in A^{p+1}$ tal que $f^{p+1}(a)=d_{B}^{p}(b)$ é um $p$-ciclo. Por fim, temos que $[a] \in$ $H^{p+1}\left(A^{*}\right)$ independe da escolha de $b \in\left(g^{p}\right)^{-1}(c)$.

Para facilitar o entendimento das provas dessas afirmações, ilustramos o diagrama de uma sequência exata curta de complexos de cadeia, onde $\partial^{*}$ aparece em destaque:

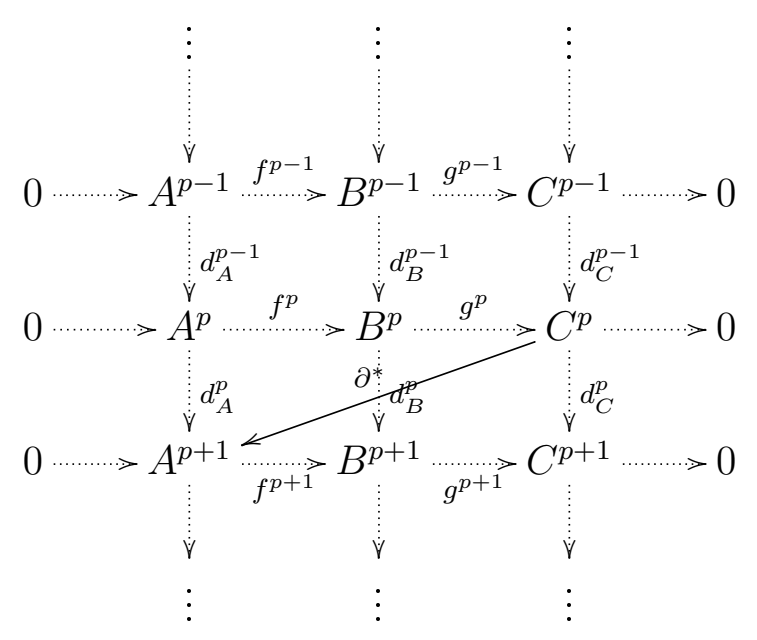

No que se segue provaremos algumas afirmações que sustentam a boa definição de $\partial^{*}$.

(i) Se $g^{p}(b)=c$ e $d_{C}^{p}(c)=0$ então $d_{B}^{p}(b) \in \operatorname{im} f^{p+1}$.

De fato, $g^{p+1} d_{B}^{p}(b)=d_{C}^{p}\left(g^{p}(b)\right)=d_{C}^{p}(c)=0$. Como a sequência é exata temos $\operatorname{ker} g^{p+1}=\operatorname{im} f^{p+1}, \operatorname{logo} d_{B}^{p}(b) \in \operatorname{im} f^{p+1}$.

(ii) Se $f^{p+1}(a)=d_{B}^{p}(b)$ então $d_{A}^{p+1}(a)=0$.

Temos $f^{p+2}\left(d_{A}^{p+1}(a)\right)=d_{B}^{p+1}\left(f^{p+1}(a)\right)=d_{B}^{p+1}\left(d_{B}^{p}(b)\right)=0$. Como $f$ é injetiva, $d_{A}^{p+1}(a)=0$.

(iii) Se $g^{p}\left(b_{1}\right)=g^{p}\left(b_{2}\right)=c$ e $f^{p+1}\left(a_{i}\right)=d_{B}^{p}\left(b_{i}\right)$, então $\left[a_{1}\right]=\left[a_{2}\right] \in H^{p+1}\left(A^{*}\right)$.

Como $g^{p}\left(b_{1}\right)=g^{p}\left(b_{2}\right)$, temos $g^{p}\left(b_{1}-b_{2}\right)=0, \operatorname{logo} b_{1}-b_{2} \in \operatorname{ker} g=\operatorname{im} f$, então $b_{1}-$ $b_{2}=f^{p}(a)$, para algum $a \in A^{p} . \operatorname{Assim} d_{B}^{p}\left(b_{1}\right)-d_{B}^{p}\left(b_{2}\right)=d_{B}^{p}\left(f^{p}(a)\right)=f^{p+1}\left(d_{A}^{p}(a)\right)$ e por fim $\left(f^{p+1}\right)^{-1}\left(d_{B}^{p}\left(b_{1}\right)\right)=\left(f^{p+1}\right)^{-1}\left(d_{B}^{p}\left(b_{2}\right)\right)+d_{A}^{p}(a)$, daí $\left[a_{1}\right]=\left[a_{2}\right]$.

Lema 2.2.3. A sequência

$$
H^{p}\left(B^{*}\right) \stackrel{g^{*}}{\longrightarrow} H^{p}\left(C^{*}\right) \stackrel{\partial^{*}}{\longrightarrow} H^{p+1}\left(A^{*}\right)
$$

é exata. 
Demonstração: Temos que

$$
\begin{aligned}
\partial^{*}\left(g^{*}([b])\right) & =\partial^{*}([g(b)]) \\
& =\left(f^{p+1}\right)^{-1}\left(d_{B}^{p}\left(g^{p}\right)^{-1}(g(b))\right) \\
& =\left(f^{p+1}\right)^{-1}\left(d_{B}^{p}(b)\right)=0
\end{aligned}
$$

pois $b$ é um $p$-ciclo. Logo im $g^{*} \subseteq \operatorname{ker} \partial^{*}$.

Por outro lado se $\partial^{*}[c]=0$, usando a sobrejeção de $g$, escolhemos $b \in B^{p}$ tal que $g^{p}(b)=c$. Como $\partial^{*}[c]=\left[\left(f^{p+1}\right)^{-1}\left(d_{B}^{p}(b)\right)\right]$, existe $a \in A^{p}$ tal que:

$$
d_{B}^{p}(b)=f^{p+1}\left(d_{A}^{p} a\right)=d_{B}^{b}\left(f^{p}(a)\right)
$$

$\operatorname{assim} d_{B}^{p}\left(b-f^{p}(a)\right)=0$, isto é $\left(b-f^{p}(a)\right)$ é um $p$-ciclo. Como $g\left(b+f^{p}(a)\right)=c$, temos $g^{*}\left[b-f^{p}(a)\right]=[c]$, ou seja, ker $\partial^{*} \subseteq \operatorname{im} g^{*}$.

Lema 2.2.4. A sequência

$$
H^{p}\left(C^{*}\right) \stackrel{\partial^{*}}{\longrightarrow} H^{p+1}\left(A^{*}\right) \stackrel{f^{*}}{\longrightarrow} H^{p+1}\left(B^{*}\right)
$$

é exata.

Demonstração: Tomemos $b \in B^{p}$ tal que $g(b)=c$. Assim:

$$
f^{*} \partial^{*}([c])=\left[f^{p+1}\left(\left(f^{p+1}\right)^{-1} d_{B}^{p}\left(g^{p}\right)^{-1}(c)\right)\right]=\left[d_{B}^{p}(b)\right]=0
$$

o que nos dá im $\partial^{*} \subseteq \operatorname{ker} f^{*}$

Reciprocamente, seja $f^{*}[a]=0$. Então $f^{p+1}(a)=d_{B}^{p}(b)$, para algum $b \in B^{p}$. Assim $d_{C}^{p}\left(g^{p}(b)\right)=g^{p+1}\left(d_{B}^{p}(b)\right)=g^{p+1}\left(f^{p+1}(a)\right)=0$, pois $\operatorname{ker} g^{p+1}=\operatorname{im} f^{p+1}$ e encerramos com $\partial^{*}\left[g^{p}(b)\right]=[a]$, ou seja, $\operatorname{ker} f^{*} \subseteq \operatorname{im} \partial^{*}$.

Teorema 2.2.5. (da sequência exata longa de homologia) Para uma sequência exata curta de complexos de cadeia $0 \longrightarrow A^{*} \stackrel{f}{\longrightarrow} B^{*} \stackrel{g}{\longrightarrow} C^{*} \longrightarrow 0$, a sequência

$$
\cdots \longrightarrow H^{p}\left(A^{*}\right) \stackrel{f^{*}}{\longrightarrow} H^{p}\left(B^{*}\right) \stackrel{g^{*}}{\longrightarrow} H^{p}\left(C^{*}\right) \stackrel{\partial^{*}}{\longrightarrow} H^{p+1}\left(A^{*}\right) \stackrel{f^{*}}{\longrightarrow} H^{p+1}\left(B^{*}\right) \longrightarrow \cdots
$$

é exata.

A prova deste teorema é a junção das provas dos três lemas anteriores. 
Definição 2.2.6. Duas aplicações de cadeia $f, g: A^{*} \longrightarrow B^{*}$ são ditas homotópicas se existir uma aplicação linear $s_{p}: A^{p} \longrightarrow B^{p-1}$, satisfazendo:

$$
d_{B}^{p-1} s_{p}+s_{p} d_{A}^{p-1}=f-g
$$

para todo $p$.

O diagrama abaixo nos dá a visão de duas aplicações em cadeia que são homotópicas:

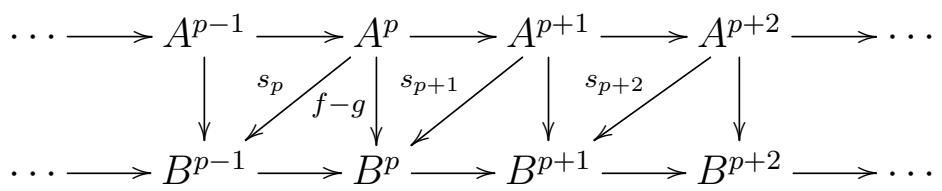

Lema 2.2.7. Duas aplicações de cadeia que são homotópicas $f, g: A^{*} \longrightarrow B^{*}$, induzem a mesma aplicação em homologia, ou seja:

$$
f *=g *: H^{p}(A) \longrightarrow H^{p}(B) .
$$

Demonstração: Tomemos $[a] \in H^{p}(A)$, então:

$$
\left(f^{*}-g^{*}\right)[a]=\left[f^{p}(a)-g^{p}(a)\right]=\left[d_{B}^{p} s_{p}(a)+s_{p} d_{A}^{p}(a)\right]=\left[d_{B}^{p} s_{p}(a)\right]=0
$$

pois $d_{A}^{p}(a)$ é um $p$-ciclo e $d_{B}^{p} s_{p}(a)$ é uma $p$-fronteira.

Observação 2.2.8. Para provar o lema de Poincaré no capítulo anterior, construímos aplicações lineares

$$
S^{p}: \Omega^{p}(U) \longrightarrow \Omega^{p-1}(U)
$$

com $d^{p-1} S^{p}+S^{p+1} d^{p}=i d$, para $p>0$, que é uma homotopia entre a id e 0. Assim temos que:

$$
i d^{*}=0^{*}: H^{p}(U) \longrightarrow H^{p}(U)
$$

Contudo, $i d^{*}=i d$ e $0^{*}=0$, então $i d=0$ em $H^{p}(U)$ e $H^{p}(U)=0$ quando $p>0$.

Lema 2.2.9. Se $A^{*}$ e $B^{*}$ são complexos de cadeia, então:

$$
H^{p}\left(A^{*} \oplus B^{*}\right)=H^{p}\left(A^{*}\right) \oplus H^{p}\left(B^{*}\right) .
$$


Demonstração: Segue diretamente dos fatos:

$$
\begin{aligned}
& \operatorname{ker} d_{A \oplus B}^{p}=\operatorname{ker} d_{A}^{p} \oplus \operatorname{ker} d_{B}^{p} \\
& \operatorname{im} d_{A \oplus B}^{p}=\operatorname{im} d_{A}^{p} \oplus \operatorname{im} d_{B}^{p}
\end{aligned}
$$

o que prova o lema. 


\section{Capítulo}

\section{A sequência de Mayer-Vietoris}

Neste capítulo introduziremos uma técnica para calcular a cohomologia de de Rham. Esta técnica é chamada de sequência de Mayer-Vietoris, onde conseguimos calcular $H^{*}\left(U_{1} \cup\right.$ $\left.U_{2}\right)$ em função de $H^{*}\left(U_{1}\right), H^{*}\left(U_{2}\right)$ e $H^{*}\left(U_{1} \cap U_{2}\right)$, onde $U_{1}$ e $U_{2}$ são abertos do $\mathbb{R}^{n}$. De um modo mais geral, somos capazes de calcular $H^{*}\left(U_{1} \cup \ldots \cup U_{n}\right)$ em função $\operatorname{dos} H^{*}\left(U_{\alpha}\right)$, onde $\alpha=\left\{i_{1}, \ldots, i_{r}\right\}$ são subconjuntos de $\{1, \ldots, n\}$ e $U_{\alpha}=U_{i_{1}} \cap \ldots \cap U_{i_{r}}$. Esta técnica, combinada com o lema de Poincaré, é capaz de produzir $H^{*}(U)$ para muitos abertos do $\mathbb{R}^{n}$. Por exemplo, se $U$ pode ser coberto por um número finito de abertos convexos $U_{i}$, então todo $U_{\alpha}$ também será convexo e $H^{*}\left(U_{\alpha}\right)$ é conhecido, pelo lema de Poincaré.

Teorema 3.0.10. Sejam $U_{1}$ e $U_{2}$ são abertos em $\mathbb{R}^{n}$ com $U=U_{1} \cup U_{2}$ e consideremos as aplicações

$$
\begin{aligned}
& i_{1}: U_{1} \longrightarrow U \\
& j_{1}: U_{1} \cap U_{2} \quad \longrightarrow \quad U_{1} \\
& i_{2}: U_{2} \longrightarrow U \\
& j_{2}: U_{1} \cap U_{2} \longrightarrow U_{2}
\end{aligned}
$$

as correspondentes inclusões. Então a sequência:

$$
0 \longrightarrow \Omega^{p}(U) \stackrel{I^{*}}{\longrightarrow} \Omega^{p}\left(U_{1}\right) \oplus \Omega^{p}\left(U_{2}\right) \stackrel{J^{*}}{\longrightarrow} \Omega^{p}\left(U_{1} \cap U_{2}\right) \longrightarrow 0
$$

é exata, onde $I^{p}(\omega)=\left(i_{1}^{*}(\omega), i_{2}^{*}(\omega)\right)$ e $J^{p}\left(\omega_{1}, \omega_{2}\right)=j_{1}^{*}\left(\omega_{1}\right)-j_{2}^{*}\left(\omega_{2}\right)$.

Demonstração: Relembremos alguns pontos já alcançados. Para uma aplicação suave 
$\phi: U \longrightarrow W$ e uma $p$-forma $\omega=\sum_{I} f_{I} d x_{I} \in \Omega^{p}(W)$, temos:

$$
\phi^{*}(\omega)=\sum_{I}\left(f_{I} \circ \phi\right) d \phi_{i_{1}} \wedge \cdots \wedge d \phi_{i_{p}}
$$

Em particular, quando $\phi$ for uma inclusão, podemos escrever $\phi_{i}=x_{i}$, assim:

$$
d \phi_{i_{1}} \wedge \cdots \wedge d \phi_{i_{p}}=d x_{i_{1}} \wedge \cdots \wedge d x_{i_{p}}
$$

e temos:

$$
\phi^{*}(\omega)=\sum_{I}\left(f_{I} \circ \phi\right) d x_{I}=\sum_{I}\left(\left.f_{I}\right|_{U}\right) d x_{I}=:\left.\omega\right|_{U}
$$

Seja $\omega \in \Omega^{p}(U)$ tal que $I^{p}(\omega)=0$. Logo $i_{1}^{*}(\omega)=i_{2}^{*}(\omega)=0$, e temos

$$
i_{1}^{*}(\omega)=\sum_{I}\left(f_{I} \circ i_{1}\right) d x_{I}=\sum_{I}\left(\left.f_{I}\right|_{U_{1}}\right) d x_{I}=0
$$

Isso ocorre se, e somente se, $\left.f_{I}\right|_{U_{1}}=0$. Usando o mesmo raciocíneo para $i_{2}^{*}$, chegamos a conclusão que tevemos ter $f_{I}=0$ para todo $x \in U$, pois $\left.f_{I}\right|_{U_{1}}=0=\left.f_{I}\right|_{U_{2}}$ e $U=U_{1} \cup U_{2}$, $\log 0 \omega=0$ e $I^{p}$ é injetora.

Mostremos agora que ker $J^{p}=\operatorname{im} I^{p}$. Seja $\omega \in \Omega^{p}(U)$, então:

$$
\begin{aligned}
J^{p} \circ I^{p}(\omega) & =j_{2}^{*} i_{2}^{*}(\omega)-j_{1}^{*} i_{1}^{*}(\omega) \\
& =j_{2}^{*}\left(\left.\omega\right|_{U_{2}}\right)-j_{1}^{*}\left(\left.\omega\right|_{U_{1}}\right) \\
& =\left.\omega\right|_{U_{1} \cap U_{2}}-\left.\omega\right|_{U_{1} \cap U_{2}}=0,
\end{aligned}
$$

ou seja, im $I^{p} \subset \operatorname{ker} J^{p}$. Tomemos agora duas $p$-formas $\omega_{1} \in \Omega^{p}\left(U_{1}\right)$ e $\omega_{2} \in \Omega^{p}\left(U_{2}\right)$,

$$
\omega_{1}=\sum_{I} f_{I} d x_{I} \quad \text { e } \quad \omega_{2}=\sum_{J} g_{J} d x_{J}
$$

tais que $\left(\omega_{1}, \omega_{2}\right) \in \operatorname{ker} J^{p}$. Assim $J^{p}\left(\omega_{1}, \omega_{2}\right)=0 \Rightarrow j_{2}^{*}\left(\omega_{2}\right)-j_{1}^{*}\left(\omega_{1}\right)=0 \Rightarrow j_{1}^{*}\left(\omega_{1}\right)=$ $\left.j_{2}^{*}\left(\omega_{2}\right) \Rightarrow \omega_{1}\right|_{U_{1} \cap U_{2}}=\left.\omega_{2}\right|_{U_{1} \cap U_{2}}$. Com isso, fica bem definida $h_{I}: U \longrightarrow \mathbb{R}^{n}$, onde:

$$
h_{I}(x)=\left\{\begin{array}{lll}
f_{I}(x) & \text { se } & x \in U_{1} \\
g_{I}(x) & \text { se } & x \in U_{2}
\end{array}\right.
$$

Notemos que $h_{I}$ é uma função suave, pois $f_{I}$ e $g_{I}$ o são e coincidem na intersecção de seus domínios. Assim, se $\omega=\sum_{I} h_{I} d x_{I}$, temos $I^{p}=\left(\omega_{1}, \omega_{2}\right)$ e com isso ker $J^{p} \subset \operatorname{im} I^{p}$. 
Resta-nos provar a sobrejetividade de $J^{p}$. Para isso usaremos uma partição da unidade, ou seja, funções suaves

$$
p_{1}: U \longrightarrow[0,1] \quad \text { e } p_{2}: U \longrightarrow[0,1]
$$

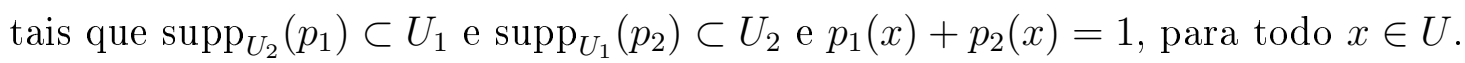

Seja $f: U_{1} \cap U_{2} \longrightarrow \mathbb{R}$ tal que $f$ é suave. Nosso objetivo é usar a partição da unidade para estender $f$ a $U_{1}$ e $U_{2}$ de forma suave. Definimos:

$$
\begin{gathered}
f_{2}(x)=\left\{\begin{array}{ccc}
-f(x) p_{1}(x) & \text { se } & x \in U_{1} \cap U_{2} \\
0 & \text { se } & x \in U_{2}-\operatorname{supp}_{U}\left(p_{1}\right)
\end{array}\right. \\
f_{1}(x)=\left\{\begin{array}{ccc}
f(x) p_{2}(x) & \text { se } & x \in U_{1} \cap U_{2} \\
0 & \text { se } & x \in U_{1}-\operatorname{supp}_{U}\left(p_{2}\right)
\end{array}\right.
\end{gathered}
$$

que são suaves e estão bem definidas pois $\operatorname{supp}_{U}\left(p_{1}\right) \subset U_{1} \cap U_{2}$ e $\operatorname{supp}_{U}\left(p_{2}\right) \subset U_{1} \cap U_{2}$. Como $p_{1}(x)+p_{2}(x)=1$, temos $f(x)=f_{1}(x)-f_{2}(x)$, se $x \in U_{1} \cap U_{2}$.

Tomemos agora $\omega \in \Omega^{p}\left(U_{1} \cap U_{2}\right)$. Podemos escrever $\omega=\sum_{I} f_{I} d x_{I}$ e aplicar a extensão apresentada acima para cada uma das funções $f_{I}: U_{1} \cap U_{2} \longrightarrow \mathbb{R}$ e obteremos $f_{I, 2}: U_{2} \longrightarrow$ $\mathbb{R}$ e $f_{I, 1}: U_{1} \longrightarrow \mathbb{R}$, ambas suaves e de tal forma que $f_{I}=f_{I, 1}-f_{I, 2}$ em $U_{1} \cap U_{2}$. Com isso obtemos duas $p$-formas $\omega_{1}=\sum_{I} f_{I, 1} d x_{I} \in \Omega^{p}\left(U_{1}\right)$ e $\omega_{2}=\sum_{I} f_{I, 2} d x_{I} \in \Omega^{p}\left(U_{2}\right)$, tais que:

$$
\begin{aligned}
J^{p}\left(\omega_{1}, \omega_{2}\right) & =j_{1}^{*}\left(\omega_{1}\right)-j_{2}^{*}\left(\omega_{2}\right) \\
& =\left.\omega_{1}\right|_{U_{1} \cap U_{2}}-\left.\omega_{2}\right|_{U_{1} \cap U_{2}} \\
& =\left.\sum_{I} f_{I, 1}\right|_{U_{1} \cap U_{2}} d x_{I}-\left.\sum_{I} f_{I, 2}\right|_{U_{1} \cap U_{2}} d x_{I} \\
& =\sum_{I}\left(\left.f_{I, 1}\right|_{U_{1} \cap U_{2}}-\left.f_{I, 2}\right|_{U_{1} \cap U_{2}}\right) d x_{I} \\
& =\sum_{I}^{I} f_{I} d x_{I}=\omega
\end{aligned}
$$

ou seja, $J^{p}$ é sobrejetora.

Teorema 3.0.11. (Mayer-Vietoris) Tomemos dois abertos $U_{1}$ e $U_{2}$ em $\mathbb{R}^{n}$ e consideremos $U=U_{1} \cup U_{2}$. Então existe uma sequência exata de cohomologia.

$$
\cdots \longrightarrow H^{p}(U) \stackrel{I^{*}}{\longrightarrow} H^{p}\left(U_{1}\right) \oplus H^{p}\left(U_{2}\right) \stackrel{J^{*}}{\longrightarrow} H^{p}\left(U_{1} \cap U_{2}\right) \stackrel{\partial^{*}}{\longrightarrow} H^{p+1}(U) \longrightarrow \cdots
$$

onde $I^{*}([\omega])=\left(i_{1}^{*}[\omega], i_{2}^{*}[\omega]\right)$ e $J^{*}\left(\left[\omega_{1}\right],\left[\omega_{2}\right]\right)=j_{1}^{*}\left[\omega_{1}\right]-j_{2}^{*}\left[\omega_{2}\right]$, são as mesmas inclusões do 
teorema anterior.

Demonstração: Consideremos a cadeia:

$$
0 \longrightarrow \Omega^{*}(U) \stackrel{I}{\longrightarrow} \Omega^{*}\left(U_{1}\right) \oplus \Omega^{*}\left(U_{2}\right) \stackrel{J}{\longrightarrow} \Omega^{*}\left(U_{1} \cap U_{2}\right) \longrightarrow 0
$$

Pelo teorema 3.0 .10 temos que esta cadeia é uma sequência exata curta de complexos de cadeia. O teorema 2.2.5 nos dá que a sequência pode ser expandida a uma sequência longa em cohomologia:

$\cdots \longrightarrow H^{p}\left(\Omega^{*}(U)\right) \stackrel{I^{*}}{\longrightarrow} H^{p}\left(\Omega^{*}\left(U_{1}\right) \oplus \Omega^{*}\left(U_{2}\right)\right) \stackrel{J^{*}}{\longrightarrow} H^{p}\left(\Omega^{*}\left(U_{1} \cap U_{2}\right)\right) \stackrel{\partial^{*}}{\longrightarrow} H^{p+1}\left(\Omega^{*}(U)\right) \longrightarrow \cdots$

e o lema 2.2 .9 nos dá a última igualdade necessária $H^{p}\left(\Omega^{*}\left(U_{1}\right) \oplus \Omega^{*}\left(U_{2}\right)\right)=H^{p}\left(\Omega^{*}\left(U_{1}\right)\right) \oplus$ $H^{p}\left(\Omega^{*}\left(U_{2}\right)\right)$.

Corolário 3.0.12. Sejam $U_{1}$ e $U_{2}$ abertos do $\mathbb{R}^{n}$ disjuntos, então:

$$
I^{*}: H^{p}\left(U_{1} \cup U_{2}\right) \longrightarrow H^{p}\left(U_{1}\right) \oplus H^{p}\left(U_{2}\right)
$$

é um isomorfismo.

Demonstração: Como $U_{1} \cap U_{2}=\varnothing$, temos $H^{p}\left(U_{1} \cap U_{2}\right)=0=H^{p-1}\left(U_{1} \cap U_{2}\right)$. Logo, pelo teorema 3.0 .11 temos:

$$
0 \longrightarrow H^{p}(U) \stackrel{I^{*}}{\longrightarrow} H^{p}\left(U_{1}\right) \oplus H^{p}\left(U_{2}\right) \longrightarrow 0
$$

assim, $I^{*}$ é bijetora, logo um isomorfismo.

Exemplo 3.0.13. Usaremos o teorema 3.0.11 para calcular a cohomologa de de Rham do plano perfurado $\mathbb{R}^{2}-\{0\}$. Tomemos

$$
\begin{aligned}
& U_{1}=\mathbb{R}^{2}-\{(x, y) \mid x \geqslant 0 ; y=0\} \\
& U_{2}=\mathbb{R}^{2}-\{(x, y) \mid x \leqslant 0 ; y=0\} .
\end{aligned}
$$

Tanto $U_{1}$ quanto $U_{2}$ são abertos extrelados do $\mathbb{R}^{2}$, logo $H^{p}\left(U_{1}\right)=H^{p}\left(U_{2}\right)=0$, para $p>0$ e $H^{0}\left(U_{1}\right)=H^{0}\left(U_{2}\right)=\mathbb{R}$. A interseção $U_{1} \cap U_{2}=\mathbb{R}^{2}-\mathbb{R}=\mathbb{R}_{+}^{2} \cup \mathbb{R}_{-}^{2}$, é a uni $\tilde{a} o$ 
disjunta de dois abertos, os semi-planos $y>0$ e $y<0$. Logo, pelo corolário anterior e pelo lema de Poincaré:

$$
H^{p}\left(U_{1} \cup U_{2}\right)=\left\{\begin{array}{ccc}
0 & \text { se } & p>0 \\
\mathbb{R} \oplus \mathbb{R} & \text { se } & p=0
\end{array}\right.
$$

Pela sequência de Mayer-Vietoris, temos:

$$
\begin{gathered}
\cdots \longrightarrow H^{p}\left(U_{1}\right) \oplus H^{p}\left(U_{2}\right) \stackrel{J^{*}}{\longrightarrow} H^{p}\left(U_{1} \cap U_{2}\right) \stackrel{\partial^{*}}{\longrightarrow} \\
H^{p+1}\left(\mathbb{R}^{2}-\{0\}\right) \stackrel{I^{*}}{\longrightarrow} H^{p+1}\left(U_{1}\right) \oplus H^{p+1}\left(U_{2}\right) \longrightarrow \cdots
\end{gathered}
$$

Onde, para $p>0$, temos:

$$
0 \longrightarrow H^{p}\left(U_{1} \cap U_{2}\right) \stackrel{\partial^{*}}{\longrightarrow} H^{p+1}\left(\mathbb{R}^{2}-\{0\}\right) \longrightarrow 0
$$

é uma sequência exata. Assim, $\partial^{*}$ é um isomorfismo e $H^{p}\left(\mathbb{R}^{2}-\{0\}\right)=0$ para $p>0$.

Quando $p=0$ temos a sequência exata:

$$
\begin{gathered}
\cdots \longrightarrow H^{-1}\left(U_{1} \cap U_{2}\right) \longrightarrow H^{0}\left(\mathbb{R}^{2}-\{0\}\right) \stackrel{I^{0}}{\longrightarrow} H^{0}\left(U_{1}\right) \oplus H^{0}\left(U_{2}\right) \stackrel{J^{0}}{\longrightarrow} \\
H^{0}\left(U_{1} \cap U_{2}\right) \stackrel{\partial^{*}}{\longrightarrow} H^{1}\left(\mathbb{R}^{2}-\{0\}\right) \stackrel{I^{1}}{\longrightarrow} H^{1}\left(U_{1}\right) \oplus H^{1}\left(U_{2}\right) \longrightarrow \cdots
\end{gathered}
$$

Como $H^{-1}(U)=0$ para qualquer aberto, em particular $H^{-1}\left(U_{1} \cap U_{2}\right)=0$ e assim, $I^{0}$ é injetiva. Como $H^{1}\left(U_{1}\right)=H^{1}\left(U_{2}\right)=0$, $\partial^{*}$ é sobrejetiva. A sequência ** é:

$$
\begin{gathered}
0 \longrightarrow H^{0}\left(\mathbb{R}^{2}-\{0\}\right) \stackrel{I^{0}}{\longrightarrow} \overbrace{H^{0}\left(U_{1}\right) \oplus H^{0}\left(U_{2}\right)}^{=\mathbb{R} \oplus \mathbb{R}} \stackrel{J^{0}}{\longrightarrow} \\
\overbrace{H^{0}\left(U_{1} \cap U_{2}\right)}^{=\mathbb{R} \oplus \mathbb{R}} \stackrel{\partial^{*}}{\longrightarrow} H^{1}\left(\mathbb{R}^{2}-\{0\}\right) \longrightarrow 0
\end{gathered}
$$

Contudo, $U=\mathbb{R}^{2}-0$ é conexo. Então $H^{0}\left(\mathbb{R}^{2}-0\right) \cong \mathbb{R}$ e, pelo fato de $I^{0}$ ser injetiva, devemos ter im $I^{0} \cong \mathbb{R}$ e, como a sequência é exata im $I^{0}=\operatorname{ker} J^{0}$, logo, $J^{0}$ tem posto 1 . Portanto:

$$
\partial^{*}: H^{0}\left(U_{1} \cap U_{2}\right) / i m J^{0} \stackrel{\cong}{\longrightarrow} H^{1}\left(\mathbb{R}^{2}-\{0\}\right)
$$


Como $H^{0}\left(U_{1} \cap U_{2}\right)=i m J^{0} \cong \mathbb{R}$, mostramos que:

$$
H^{p}\left(\mathbb{R}^{2}-\{0\}\right)=\left\{\begin{array}{ccc}
0 & \text { se } & p \geqslant 2 \\
\mathbb{R} & \text { se } & p=1 \text { ou } p=0
\end{array}\right.
$$

Observação 3.0.14. A cohomologa de de Rham $H^{1}\left(\mathbb{R}^{2}-\{0\}\right)$ tem um gerador conhecido. É a forma elemento de ângulo:

$$
\omega=\frac{-y d x+x d y}{x^{2}+y^{2}}
$$

Um estudo sobre este gerador pode ser encontrado no item [2] da bibliografia, página 191, teorema 2.

Teorema 3.0.15. Seja $U \subset \mathbb{R}^{n}$. Caso existam abertos convexos $U_{1}, \ldots, U_{r}$ tais que $U=U_{1} \cup \ldots \cup U_{r}$, então $H^{p}(U)$ é finitamente gerado.

Demonstração: Provaremos este fato por de indução sobre $r$.

O caso $r=1$ está coberto pelo lema de Poincaré. Assumiremos verdadeiro para $r-1$ e sejam $V=U_{1} \cup \ldots \cup U_{r-1}$ e $U=V \cup U_{r}$. O teorema anterior nos dá a sequência exata:

$$
H^{p-1}\left(V \cap U_{r}\right) \stackrel{\partial^{*}}{\longrightarrow} H^{p}(U) \stackrel{I^{*}}{\longrightarrow} H^{p}(V) \oplus H^{p}\left(U_{r}\right)
$$

o que nos leva a:

$$
0 \longrightarrow \operatorname{im} \partial^{*} \stackrel{\partial^{*}}{\longrightarrow} H^{p}(U) \stackrel{I^{*}}{\longrightarrow} \operatorname{im} I^{*} \longrightarrow 0
$$

que também é exata. O lema 2.1.1 diz que $H^{p}(U)=\operatorname{im} \partial^{*} \oplus \operatorname{im} I^{*}$. Como $V \cap U_{r}=$ $\left(U_{1} \cap U_{r}\right) \cup \ldots \cup\left(U_{r-1} \cap U_{r}\right)$, temos que tanto $V$ quanto $V \cap U_{r}$ são a união de $r-1$ abertos convexos. Então, pela hipótese de indução, temos que $H^{p-1}\left(V \cap U_{r}\right), H^{p}(V)$ e $H^{p}\left(U_{r}\right)$ são finitamente gerados e, portanto, $H^{p}(U)$ também. 


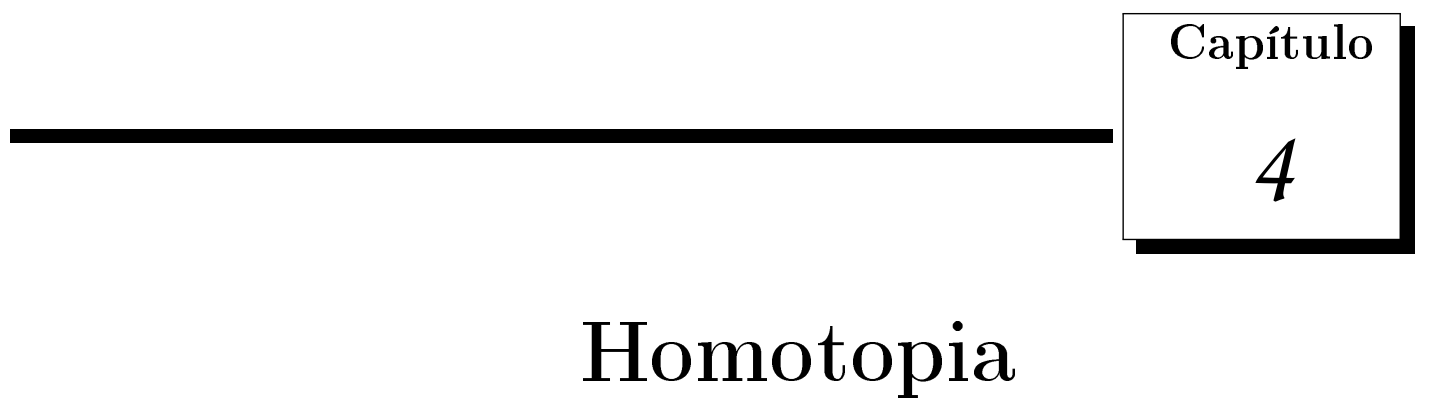

Neste capítulo mostraremos que a cohomologia de de Rham é funtorial na categoria das aplicações diferenciáveis entre espaços euclidianos e calcularemos $H^{*}\left(\mathbb{R}^{n}-\{0\}\right)$.

\subsection{O funtor de cohomologia}

Definição 4.1.1. Duas aplicações contínuas $f_{0}: X \longrightarrow Y$ e $f_{1}: X \longrightarrow Y$ entre espaços topológicos são chamados de homotópicas se existir uma aplicação contínua:

$$
F: X \times[0,1] \longrightarrow Y
$$

tal que $F(x, 0)=f_{0}(x)$ e $F(x, 1)=f_{1}(x)$, para todo $x \in X$.

Denotaremos a homotopia entre duas aplicações por $f_{0} \simeq f_{1}$, e $F$ é chamada de homotopia de $f_{0}$ a $f_{1}$. Podemos pensar em $F$ como a família das aplicações contínuas $f_{t}: X \longrightarrow Y$ com $0 \leqslant t \leqslant 1$ dada por $f_{t}(x)=F(x, t)$, que deformam $f_{0}$ em $f_{1}$.

Lema 4.1.2. Homotopia é uma relação de equivalência.

Demonstração: Ora, se $F$ é uma homotopia de $f_{0}$ a $f_{1}$, podemos definir a homotopia de $f_{1}$ a $f_{0}$ por $G(x, t)=F(x, 1-t)$, para $0 \leqslant t \leqslant 1$, ou seja, se $f_{0} \simeq f_{1}$ então, $f_{1} \simeq f_{0}$. 
Se $f_{0} \simeq f_{1}$ por $F$ e $f_{1} \simeq f_{2}$ por $G$ definimos:

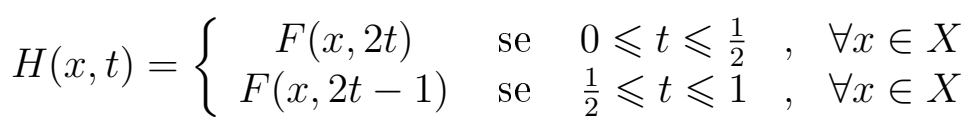

então $f_{0} \simeq f_{2}$ por $H$.

Finalmente temos $f_{0} \simeq f_{0}$ via $F(x, t)=f_{0}(x), 0 \leqslant t \leqslant 1$ e qualquer $x \in X$.

Lema 4.1.3. Sejam $X, Y$ e $Z$ espaços topológicos e

$$
\begin{array}{ll}
f_{0}: X \longrightarrow Y & g_{0}: Y \longrightarrow Z \\
f_{1}: X \longrightarrow Y & g_{1}: Y \longrightarrow Z
\end{array}
$$

aplicações contínuas. Se $f_{0} \simeq f_{1}$ e $g_{0} \simeq g_{1}$, então $g_{0} \circ f_{0} \simeq g_{1} \circ f_{1}$.

Demonstração: Consideremos:

$$
F(x, t), 0 \leqslant t \leqslant 1, \forall x \in X \quad \text { e } \quad G(y, t), 0 \leqslant t \leqslant 1, \forall y \in Y
$$

onde $F$ é a homotopia de $f_{0}$ para $f_{1}$ e $G$ a de $g_{0}$ para $g_{1}$ e definimos:

$$
H(x, t)=G(F(x, t), t), 0 \leqslant t \leqslant 1, \forall x \in X .
$$

Notemos que $H$ é contínua por ser a composição de funções contínuas. Além disso:

$$
H(x, 0)=G(F(x, 0), 0)=G\left(f_{0}(x), 0\right)=g_{0} \circ f_{0}(x)
$$

e

$$
H(x, 1)=G(F(x, 1), 1)=G\left(f_{1}(x), 1\right)=g_{1} \circ f_{1}(x) .
$$

Logo, $g_{0} \circ f_{0} \simeq g_{1} \circ f_{1}$ por $H$.

Definição 4.1.4. Uma aplicação contínua $f: X \longrightarrow Y$ é chamada equivalência de homotopia se existir uma aplicação continua $g: Y \longrightarrow X$ tal que $f \circ g \simeq i d_{X}$ e $g \circ f \simeq i d_{Y}$. Neste caso, g é chamada de homotopia inversa de $f$.

Definição 4.1.5. Dois espaço topológicos $X$ e $Y$ são ditos homotopicamente equivalentes (ou simplesmente homotópicos) quando existir uma equivalência de homotopia entre eles. 
Definição 4.1.6. Um espaço topológico $X$ é dito contrátil se for homotópico a um ponto, o que é o mesmo que dizer que $i d_{X}$ é homotópica a uma função constante.

Definição 4.1.7. As classes de equivalência definidas pela relação de equivalência de homotopia são chamadas de tipos homotópicos.

Exemplo 4.1.8. Seja $Y \subseteq \mathbb{R}^{m}$ com a topologia induzida por $\mathbb{R}^{m}$. Se para duas aplicações contínuas $f_{0}: X \longrightarrow Y$ e $f_{1}: X \longrightarrow Y$, o segmento de reta que liga $f_{0}(x)$ a $f_{1}(x)$ estiver contido em $Y$ para todo $x \in X$, podemos definir uma homotopia de $f_{0}$ para $f_{1}$, da seguinte forma:

$$
\begin{aligned}
F: X \times[0,1] & \longrightarrow & Y \\
(x, t) & \longmapsto & (1-t) f_{0}(x)+t f_{1}(x) .
\end{aligned}
$$

Em particular este resultado também mostra que todo conjunto estrelado em $\mathbb{R}^{n}$ é contrátil.

Lema 4.1.9. Se $U, V$, são abertos em espaços euclidianos, então:

(i) Toda aplicação contínua $h: U \longrightarrow V$ é homotópica a uma aplicação diferenciável;

(ii) Para duas aplicações diferenciáveis $f_{0}: U \longrightarrow V$ e $f_{1}: U \longrightarrow V$ homotópicas, existe uma aplicação diferenciácel $F: U \times \mathbb{R} \longrightarrow V \operatorname{com} F(x, 0)=f_{0}(x)$ e $F(x, 1)=f_{1}(x)$, para todo $x \in U$ ( $F$ é chamada de homotopia suave de $f_{0}$ para $f_{1}$ ).

Demonstração: Pelo lema A no anexo deste trabalho, podemos aproximar $h$ por uma função $f$ que seja suave, de forma que todo segmento que ligue $h(x)$ à $f(x)$ esteja contido em $V$. Assim, pela mesma $F$ do exemplo anterior, $h \simeq f$.

Para (ii), consideremos $G$ a homotopia de $f_{0}$ para $f_{1}$ e $\psi: \mathbb{R} \longrightarrow[0,1]$ uma função suave tal que

$$
\begin{cases}\psi(t)=0 & \text { se } \quad t \leqslant \frac{1}{3} \\ \psi(t)=1 & \text { se } \quad t \geqslant \frac{2}{3}\end{cases}
$$

Construímos:

$$
\begin{aligned}
H: U \times \mathbb{R} & \longrightarrow V \\
(x, t) & \longmapsto H(x, t)=G(x, \psi(t))
\end{aligned}
$$

como $H(x, t)=f_{0}(x)$ para $t \leqslant \frac{1}{3}$ e $H(x, t)=f_{1}(x)$ para $t \geqslant \frac{2}{3}$, $H$ é suave em $U \times$ $\left(-\infty, \frac{1}{3}\right) \cup U \times\left(\frac{2}{3}, \infty\right)$. Assim, o lema $\mathrm{A}$ do anexo permite que aproximemos $H$ por uma aplicação suave $F: U \times \mathbb{R} \longrightarrow V$ tal que, $F$ e $V$ tenham a mesma restrição em $U \times[0,1]$. Assim, para todo $x \in U$, temos $F(x, 0)=H(x, 0)=f_{0}(x)$ e $F(x, 1)=H(x, 1)=f_{1}(x)$. 
Teorema 4.1.10. Se $f, g: U \longrightarrow V$ são aplicações suaves e $f \simeq g$, então as aplicações induzidas

$$
f^{*}, g^{*}: \Omega^{*}(V) \longrightarrow \Omega^{*}(U)
$$

são homotopias de cadeia, como na definição 2.2.6.

Demonstração: Nesta demonstração seguiremos um roteiro parecido com a prova do lema de Poincaré (1.3.2). Toda $p$-forma pode ser escrita como:

$$
\omega=\sum_{I} f_{I}(x, t) d x_{I}+\sum_{J} g_{J}(x, t) d t \wedge d x_{J}
$$

Se $\phi_{0}: U \longrightarrow U \times \mathbb{R}$ é a inclusão $\phi_{0}(x)=(x, 0)$, então:

$$
\phi_{0}^{*}(\omega)=\sum_{I} f_{I}(x, 0) d \phi_{I}=\sum_{I} f_{I}(x, 0) d x_{I}
$$

isso porque $\phi_{0}^{*}\left(d t \wedge d x_{j}\right)=0$, pois $\phi$ é constante, como no exemplo 1.2.4. Analogamente, para $\phi_{1}=(x, 1)$ temos:

$$
\phi_{1}^{*}(\omega)=\sum_{I} f_{I}(x, 1) d \phi_{I}=\sum_{I} f_{I}(x, 1) d x_{I}
$$

Usaremos a mesma aplicação usada no lema de Poincaré.

$$
\widehat{S}_{p}: \Omega^{p}(U \times \mathbb{R}) \longrightarrow \Omega^{p-1}(U)
$$

que satisfaz:

$$
\left(d \widehat{S}_{p}+\widehat{S}_{p+1} d\right)(\omega)=\phi_{1}^{*}(\omega)-\phi_{0}^{*}(\omega)=\sum_{I} f_{I}(x, 1) d x_{I}-\sum_{I} f_{I}(x, 0) d x_{I}
$$

Consideremos a composição $U \stackrel{\phi_{0}}{\longrightarrow} U \times \mathbb{R} \stackrel{F}{\longrightarrow} V$, onde $F$ é a homotopia entre $f$ e $g$. Então $F \circ \phi_{0}=f$ e $F \circ \phi_{1}=g$. Definimos:

$$
\begin{aligned}
S_{p}: \Omega^{p}(V) & \longrightarrow \Omega^{p-1}(U) \\
\omega & \longmapsto S_{p}(\omega)=\widehat{S}_{p} \circ F^{*}(\omega) .
\end{aligned}
$$

Com isso temos $d S_{p}+S_{p+1} d=g^{*}-f^{*}$. De fato, usando a equação $\left(*\right.$ aplicada a $F^{*}$, 
obtemos:

$$
\begin{aligned}
d \widehat{S}_{p} F^{*}(\omega)+\widehat{S}_{p+1} d F^{*}(\omega) & =\phi_{1}^{*} F^{*}(\omega)-\phi_{0}^{*} F^{*}(\omega)=\left(F \circ \phi_{1}\right)^{*}(\omega)-\left(F \circ \phi_{0}\right)^{*}(\omega) \\
& =g^{*}(\omega)-f^{*}(\omega)
\end{aligned}
$$

além disso, $\widehat{S}_{p+1} d F^{*}(\omega)=\widehat{S}_{p+1} F^{*} d(\omega)=S_{p+1} d(\omega)$, pois $F$ é uma aplicação de cadeia. Logo $S_{p}$ satisfaz as condições desejadas e $f^{*}$ e $g^{*}$ são homotopias de cadeia.

Sendo assim, o lema 2.2.7 nos diz que $f^{*}=g^{*}: H^{p}(V) \longrightarrow H^{p}(U)$. Para uma aplicação contínua $\phi: U \longrightarrow V$, podemos encontrar uma aplicação suave $f: U \longrightarrow V$ com $\phi \simeq f$, pelo lema 4.1.9 item (i). Os lemas 2.2.7 e o 4.1.2, junto com o teorema anterior nos mostram quem $f^{*}: H^{p}(V) \longrightarrow H^{p}(U)$ independe da escolha da $f$. Então, podemos definir:

$$
\phi^{*}=H^{p}(\phi): H^{p}(V) \longrightarrow H^{p}(U)
$$

pondo $\phi^{*}=f^{*}$, onde $f: U \longrightarrow V$ é uma aplição suave homotópica a $\phi$.

Teorema 4.1.11. Para $U, V$ e $W$ espaços euclidianos e $p \in \mathbb{Z}$, temos:

(i) Se $\phi_{0}, \phi_{1}: U \longrightarrow V$ são aplicações contínuas e homotópicas, então

$$
\phi_{0}^{*}=\phi_{1}^{*}: H^{p}(V) \longrightarrow H^{p}(U)
$$

(ii) $S e \phi: U \longrightarrow V$ e $\psi: V \longrightarrow W$ são continuas, então

$$
(\psi \circ \phi)^{*}=\phi^{*} \circ \psi^{*}: H^{p}(W) \longrightarrow H^{p}(U)
$$

(iii) Se a aplicação contínua $\phi: U \longrightarrow V$ é uma equivalência de homotopia, então

$$
\phi^{*}: H^{p}(U) \longrightarrow H^{p}(V)
$$

é um isomorfismo.

Demonstração: Podemos tomar $f: U \longrightarrow V$ suave de tal forma que $f \simeq \phi_{0}$ e $f \simeq \phi_{1}$. Logo, pelo lema 4.1.2, $\phi_{0}$ e $\phi_{1}$ fazem parte da mesma classe de equilavência, logo $\phi_{0}^{*}=\phi_{1}^{*}$.

Caso $\phi$ e $\psi$ sejam suaves, sabemos que $\Omega^{p}(\psi \circ \phi)=\Omega^{p}(\phi) \circ \Omega^{p}(\psi)$. No caso geral, pelo lema 4.1 .9 podemos tomar $f: U \longrightarrow V$ e $g: V \longrightarrow W$ suaves e tais que $f \simeq \phi$ e $g \simeq \psi$. 
Assim, pelo lema 4.1.3, temos $\psi \circ \phi \simeq f \circ g$, e usando o lema 2.2.7. $(\psi \circ \phi)^{*}=(g \circ f)^{*}=$ $f^{*} \circ g^{*}=\phi^{*} \circ \psi^{*}$ e segue (ii).

Para (iii) basta considerar $\psi$ a homotopia inversa de $\phi$. Assim $\psi \circ \phi \simeq \operatorname{id}_{U}$ e $\phi \circ \psi \simeq \operatorname{id}_{V}$. Então $\psi^{*}: H^{p}(U) \longrightarrow H^{p}(V)$ é a inversa de $\phi^{*}: H^{p}(V) \longrightarrow H^{p}(U)$.

Este resultado nos mostra que $H^{p}(U)$ só depende do tipo de homotopia de $U$. Em particular, temos:

Corolário 4.1.12. (Invariância topológica): Um homeomorfismo $h: U \longrightarrow V$ entre abertos euclidianos induz um isomorfismo

$$
h^{*}: H^{p}(V) \longrightarrow H^{p}(U)
$$

para todo $p$.

Demonstração: Considerando no item (iii) do teorema anterior $h^{-1}: V \longrightarrow U$ a homotopia inversa de $h$, segue o resultado.

Corolário 4.1.13. Se $U \subseteq \mathbb{R}^{n}$ é um aberto contrátil, então $H^{p}(U)=0$ quando $p>0$ e $H^{0}(U)=\mathbb{R}$

Demonstração: Lembremos que um conjunto é contrátil se, e somente se, a identidade é homotópica a uma aplicação constante. Então, tomemos $F: U \times[0,1] \longrightarrow V$ a homotopia entre $f_{0}=$ id e $f_{1}$ uma aplicação constante com valor $x_{0}$.

Para qualquer $x \in U, F(x, t)$ define uma curva contida em $V$, que conecta $x$ a $x_{0}$, logo $U$ é conexo, e pelo lema $1.2 .2 H^{0}(U)=\mathbb{R}$.

Se $p>0$, então $\Omega^{p}\left(f_{1}\right): \Omega^{p}(U) \longrightarrow \Omega^{p}(U)$ é a aplicação nula e, pelo teorema 4.1.11 item (i), temos $\operatorname{id}_{H^{p}(U)}=f_{0}^{*}=f_{1}^{*}=0, \operatorname{logo}, H^{p}(U)=0$.

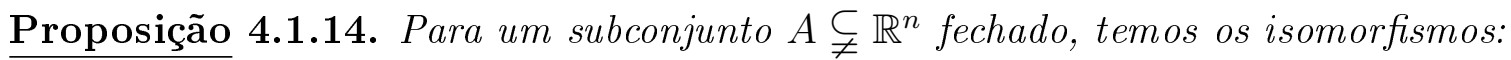

$$
\begin{aligned}
H^{p+1}\left(\mathbb{R}^{n+1}-A\right) & \cong H^{p}\left(\mathbb{R}^{n}-A\right), p \geqslant 1 \\
H^{1}\left(\mathbb{R}^{n+1}-A\right) & \cong H^{0}\left(\mathbb{R}^{n}-A\right) / \mathbb{R} \cdot 1 \\
H^{0}\left(\mathbb{R}^{n+1}-A\right) & \cong \mathbb{R}
\end{aligned}
$$

onde identificaremos $\mathbb{R}^{n}$ como o subespaço $\mathbb{R}^{n} \times\{0\}$ de $\mathbb{R}^{n+1}$ e $\mathbb{R} \cdot 1$ denota o subespaço de dimensão 1 das funções constantes. 
Demonstração: Definimos os abertos de $\mathbb{R}^{n+1}=\mathbb{R}^{n} \times \mathbb{R}$ :

$$
\begin{aligned}
& U_{1}=\mathbb{R}^{n} \times(0, \infty) \cup\left(\mathbb{R}^{n}-A\right) \times(-1, \infty) \\
& U_{2}=\mathbb{R}^{n} \times(-\infty, 0) \cup\left(\mathbb{R}^{n}-A\right) \times(-\infty, 1)
\end{aligned}
$$

Então $U_{1} \cup U_{2}=\mathbb{R}^{n+1}-A$ e $U_{1} \cap U_{2}=\mathbb{R}^{n}-A \times(-1,1)$. Tomemos $\phi: U_{1} \longrightarrow U_{2}$ dada por adicionar 1 a $(n+1)$-ésima coordenada. Para $x \in U_{1}$, temos que o segmento de reta entre $x$ e $\phi(x)$ e de $\phi(x)$ para algum ponto fixado em $\mathbb{R}^{n} \times(0, \infty)$ esta contido em $U_{1}$. Como no exemplo 4.1.8, podemos tomar homotopias de id para $\phi$ e de $\phi$ para uma aplicação constante. Logo, $U_{1}$ é contrátil. Analogamente, $U_{2}$ também é contrátil. Logo $H^{p}\left(U_{1}\right)$ e $H^{p}\left(U_{2}\right)$ são descritos no corolário 4.1.13.

Seja pr a projeção de $U_{1} \cap U_{2}$ em $\mathbb{R}^{n}-A$. Definimos $i: \mathbb{R}^{n}-A \longrightarrow U_{1} \cap U_{2}$ por $i(y)=(y, 0)$. Assim $i \circ \mathrm{pr} \simeq \operatorname{id}_{U_{1} \cap U_{2}}$. Pelo teorema 4.1.11 item (iii), concluímos que:

$$
\operatorname{pr}^{*}: H^{p}\left(\mathbb{R}^{n}-A\right) \longrightarrow H^{p}\left(U_{1} \cap U_{2}\right)
$$

é um isomorfirsmo para todo $p$. E o teorema 3.0.11 nos dá o isomorfismo

$$
\partial^{*}: H^{p}\left(U_{1} \cap U_{2}\right) \longrightarrow H^{p+1}\left(\mathbb{R}^{n+1}-A\right)
$$

para $p \geqslant 1$. Então a composição de $\operatorname{pr}^{*} \operatorname{com} \delta^{*}$ nos dá a primeira parte da prova da proposição.

Consideremos a sequência exata:

$$
0 \longrightarrow H^{0}\left(\mathbb{R}^{n+1}-A\right) \stackrel{I^{*}}{\longrightarrow} H^{0}\left(U_{1}\right) \oplus H^{0}\left(U_{2}\right) \stackrel{J^{*}}{\longrightarrow} H^{0}\left(U_{1} \cap U_{2}\right) \stackrel{\partial^{*}}{\longrightarrow} H^{1}\left(\mathbb{R}^{n+1}-A\right) \longrightarrow 0
$$

Um elemento de $H^{0}\left(U_{1}\right)$ ou $H^{0}\left(U_{2}\right)$ é uma função constante. Logo, um elemento de $H^{0}\left(U_{1}\right) \oplus H^{0}\left(U_{2}\right)$ é um par de funções constantes com valores $a_{1}$ e $a_{2}$. Sua imagem por $J^{*}$, pelo teorema 3.0 .11 , é constante em $U_{1} \cap U_{2}$ com valor $a_{1}-a_{2}$, e assim ker $\partial^{*}=\operatorname{im} J^{*}=\mathbb{R} \cdot 1$, e obtemos o isomorfismo:

$$
H^{1}\left(\mathbb{R}^{n+1}-A\right) \cong \frac{H^{0}\left(U_{1} \cap U_{2}\right)}{\mathbb{R} \cdot 1} \cong \frac{H^{0}\left(\mathbb{R}^{n+1}-A\right)}{\mathbb{R} \cdot 1}
$$

$\operatorname{como} \operatorname{dim}\left(\operatorname{im} I^{*}\right)=\operatorname{dim}\left(\operatorname{ker} \partial^{*}\right)=1, H^{0}\left(\mathbb{R}^{n+1}-A\right) \cong \mathbb{R}$

Corolário 4.1.15. Nas mesmas condições da proposição anterior, temos o difeomorfismo 
$R: \mathbb{R}^{n+1}-A \longrightarrow \mathbb{R}^{n+1}-A$, definido por:

$$
\left(x_{1}, \ldots, x_{n}, x_{n+1}\right) \longmapsto R\left(x_{1}, \ldots, x_{n}, x_{n+1}\right)=\left(x_{1}, \ldots, x_{n},-x_{n+1}\right) .
$$

A aplicação induzida

$$
R^{*}: H^{p+1}\left(\mathbb{R}^{n+1}-A\right) \longrightarrow H^{p+1}\left(\mathbb{R}^{n+1}-A\right)
$$

é a multiplicação por -1 .

Demonstração: Na notação do teorema anterior, teremos:

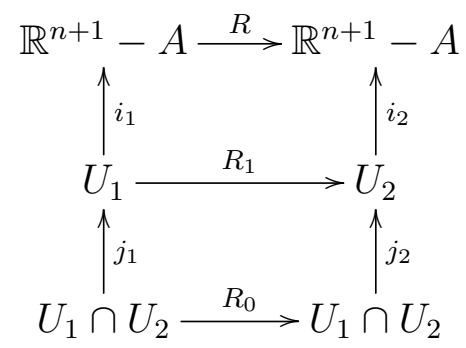

e

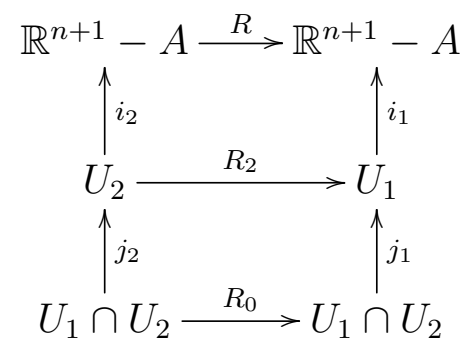

onde $R_{0}, R_{1}$ e $R_{2}$ são as restrições de $R$. Vimos que $\partial^{*}: H^{p}\left(U_{1} \cap U_{2}\right) \longrightarrow H^{p}\left(\mathbb{R}^{n+1}-A\right)$ é sobrejetora. Então, é suficiente provar que $R^{*} \circ \partial^{*}([\omega])=-\partial^{*}([\omega])$, para uma $p$-forma fechada $\omega$ em $U_{1} \cap U_{2}$.

Usando o teorema 3.0.10 podemos tomar $\omega_{1} \in \Omega^{p}\left(U_{1}\right)$ e $\omega_{2} \in \Omega^{p}\left(U_{2}\right) \operatorname{com} \omega=$ $j_{1}^{*}\left(\omega_{1}\right)-j_{2}^{*}\left(\omega_{2}\right)$. A definição 2.2 .2 de $\partial^{*}$ mostra que $\partial^{*}([\omega])=[\tau]$, onde $\tau \in \Omega^{p+1}\left(\mathbb{R}^{n+1}-A\right)$ é determinado por $i_{1}^{*}([\tau])=d \omega_{1}$ e $i_{2}^{*}([\tau])=d \omega_{2}$. Além disso:

$$
\begin{aligned}
-R_{0}^{*} \omega & =R_{0}^{*} \circ j_{2}^{*}\left(\omega_{2}\right)-R_{0}^{*} \circ j_{1}^{*}\left(\omega_{1}\right)=j_{1}^{*}\left(R_{1}^{*} \circ \omega_{2}\right)-j_{2}^{*}\left(R_{2}^{*} \circ \omega_{1}\right) \\
i_{1}^{*}\left(R^{*} \tau\right) & =R_{1}^{*}\left(i_{2}^{*} \tau\right)=R_{1}^{*}\left(d \omega_{2}\right)=d\left(R_{1}^{*} \omega_{2}\right) \\
i_{2}^{*}\left(R^{*} \tau\right) & =R_{2}^{*}\left(i_{1}^{*} \tau\right)=R_{2}^{*}\left(d \omega_{1}\right)=d\left(R_{2}^{*} \omega_{1}\right)
\end{aligned}
$$


Essas equações e a definição de $\partial^{*}$ nos dão que

$$
\begin{aligned}
\partial^{*}\left(-\left[R_{0}^{*} \omega\right]\right) & =\left[\left(I^{*}\right)^{-1}\left(d\left(J^{*}\left(-R^{*} \circ \omega\right)\right)\right)\right] \\
& =\left[\left(I^{*}\right)^{-1}\left(d\left(j_{1}^{*}\left(R_{1}^{*} \omega_{2}\right)-j_{2}^{*}\left(R_{2}^{*} \omega_{1}\right)\right)\right)\right] \\
& =\left[\left(I^{*}\right)^{-1}\left(d\left(R_{1}^{*} \omega_{2}\right)-d\left(R_{2}^{*} \omega_{1}\right)\right)\right]=\left[R^{*} \tau\right]
\end{aligned}
$$

Para a projeção pr : $U_{1} \cap U_{2} \longrightarrow \mathbb{R}^{n}-A$, temos que pr $\circ R_{0}=$ pr, e portanto:

$$
H^{p}\left(\mathbb{R}^{n}-A\right) \stackrel{\mathrm{pr}^{*}}{\longrightarrow} H^{p}\left(U_{1} \cap U_{2}\right) \stackrel{R_{0}^{*}}{\longrightarrow} H^{p}\left(U_{1} \cap U_{2}\right)
$$

é a própria pr* Como pr* é isomorfismo, $R_{0}^{*}$ tem que ser a identidade em $U_{1} \cap U_{2}$. Assim, o lado esquerdo da equação (*) é $\partial^{*}[\omega]$, o que finaliza a demonstração.

Teorema 4.1.16. Para $n \geqslant 2$, temos os isomorfismos:

$$
H^{p}\left(\mathbb{R}^{n}-\{0\}\right)=\left\{\begin{array}{ccc}
\mathbb{R}, & \text { se } \quad p=0, n-1 \\
0, & \text { caso contrário }
\end{array}\right.
$$

Demonstração: Por indução sobre $n$. O caso $n=2$ já foi feito no exemplo 3.0.13. Suponhamos que $H^{p}\left(\mathbb{R}^{k-1}-\{0\}\right)$ satisfaz as condições impostas, ora, como $\{0\}$ é um conjunto fechado, pelo lema $H^{p}\left(\mathbb{R}^{k-1}-\{0\}\right) \simeq H^{p+1}\left(\mathbb{R}^{k}-\{0\}\right)$, o que encerra a demonstração.

Observação 4.1.17. Um gerador conhecido para $H^{p}\left(\mathbb{R}^{n}-\{0\}\right)$ é a forma elemento de ângulo sólido:

$$
\omega(x)=\frac{1}{|x|^{n}} \sum_{i=1}^{n}(-1)^{i+1} x_{i} d x_{1} \wedge \ldots \wedge \widehat{d x}_{i} \wedge \ldots \wedge d x_{n} .
$$

Apresentado no item [2] da bibliografia, página 425, exemplo 17.

Uma matriz $A$ invertível $n \times n$, define um isomorfismo linear $\mathbb{R}^{n} \longrightarrow \mathbb{R}^{n}$, e um difeomorfismo $f_{A}: \mathbb{R}^{n}-\{0\} \longrightarrow \mathbb{R}^{n}-\{0\}$.

Lema 4.1.18. Para cada $n \geqslant 2$, a aplicação induzida

$$
f_{A}^{*}: H^{n-1}\left(\mathbb{R}^{n}-\{0\}\right) \longrightarrow H^{n-1}\left(\mathbb{R}^{n}-\{0\}\right)
$$

é a multiplicação por $\frac{\operatorname{det} A}{|\operatorname{det} A|} \in\{-1,1\}$ 
Demonstração: Seja $B$ a matriz obtida a partir da matriz $A$, trocando a linha $r$ pela soma das linhas $r$ e $c$ vezes a linha $s$, onde $r \neq s$ e $c \in \mathbb{R}$. Assim

$$
B=\left(I+c E_{r, s}\right) A
$$

onde $I$ é a matriz identidade e $E_{r, s}$ a matriz com as entradas iguais a 1 nas linha $r$ e coluna $s$ e 0 nas demais entradas. Assim, a homotopia que leva $f_{A}$ a $f_{B}$ é definida pela matriz:

$$
\left(I+t c E_{r, s}\right) A \quad 0 \leqslant t \leqslant 1
$$

Do teorema 4.1.11 segue que $f_{A}^{*}=f_{B}^{*}$. Além $\operatorname{disso} \operatorname{det} A=\operatorname{det} B$. Por operações elementares com a matriz $A$, ela pode ser transformada em $\operatorname{diag}(1, \ldots, 1, d)$, onde $d=$ $\operatorname{det} A$. Assim, é suficiente provar para matrizes diagonais. As matrizes:

$$
\operatorname{diag}\left(1, \ldots, 1, \frac{|d|^{t} d}{|d|}\right) \quad 0 \leqslant t \leqslant 1
$$

produz uma homotopia, o que reduz nosso problema nos casos $A=(1, \ldots, 1, \pm 1)$, então $A$ será a identidade ou a aplicação $R$ do corolário 4.1.15, o que encerra a demonstração.

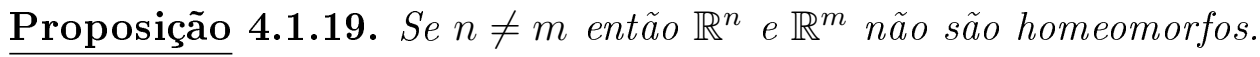

Demonstração: Podemos assumir que um possível homeomorfismo de $\mathbb{R}^{n}$ em $\mathbb{R}^{m}$ levaria 0 em 0 , caso contrário bastaria uma translação para que isso acontecesse. Este homeomorfismo induziria um homeomorfismo entre $\mathbb{R}^{n}-\{0\}$ e $\mathbb{R}^{m}-\{0\}$. Então:

$$
H^{p}\left(\mathbb{R}^{n}-\{0\}\right) \cong H^{p}\left(\mathbb{R}^{m}-\{0\}\right)
$$

para todo $p$, o que gera o absurdo. 


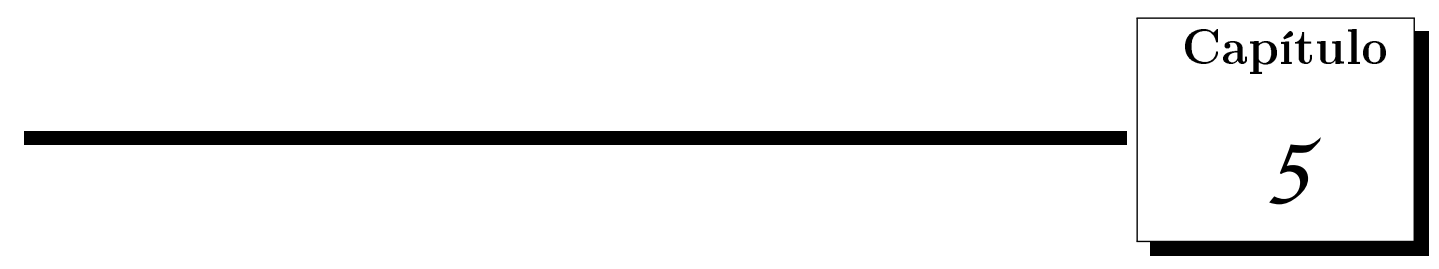

\section{Aplicações da Cohomologia de de Rham}

Neste capítulo apresentaremos aplicações da cohomologia de de Rham.

\subsection{Primeiros resultados}

Neste capítulo usaremos as notações $D^{n}=\left\{x \in \mathbb{R}^{n}\right.$ tal que $\left.\|x\| \leqslant 1\right\}$, a bola de raio um e $S^{n-1}=\left\{x \in \mathbb{R}^{n}\right.$ tal que $\left.\|x\|=1\right\}$ a esfera de raio 1 .

Lema 5.1.1. Não existe aplicação contínua $g: D^{n} \longrightarrow S^{n-1}$ tal que $\left.g\right|_{S^{n-1}}=i d_{S^{n-1}}$.

Demonstração: Vamos assumir que tal aplicação exista para $n \geqslant 2$. Consideremos a aplicação:

$$
\begin{array}{ccc}
r: \mathbb{R}^{n}-\{0\} & \longrightarrow \mathbb{R}^{n}-\{0\} \\
x & \longmapsto & \frac{x}{\|x\|} .
\end{array}
$$

Temos $\operatorname{id}_{\mathbb{R}^{n}-\{0\}} \simeq r$, pois $\mathbb{R}^{n}-\{0\}$ sempre conterá segmento de reta entre $x$ e $r(x)$, como no exemplo 4.1.8. Consideremos $H(x, t)=g(t \cdot r(x)), 0 \leqslant t \leqslant 1$, que é uma homotopia entre $r$ e uma aplicação constante. Logo $\mathbb{R}^{n}-\{0\}$ seria contrátil. Pelo corolário 4.1.13 $H^{n-1}\left(\mathbb{R}^{n}-\{0\}\right)=0$, o que contradiz o teorema 4.1.16. Se $n=1, D^{1}$ é conexo e $S^{0}=\{ \pm 1\}$ não é conexo.

Teorema 5.1.2. (Teorema do ponto fixo de Brouwer, 1912) Toda aplicação continua $f: D^{n} \longrightarrow D^{n}$ tem um ponto fixo. 
Demonstração: Suponhamos que $f(x) \neq x$ para todo $x \in D^{n}$. Assim, podemos definir o ponto $g(x) \in S^{n-1}$ como o ponto de interseção de $S^{n-1}$ com a semi-reta que liga $f(x)$ e $x$.

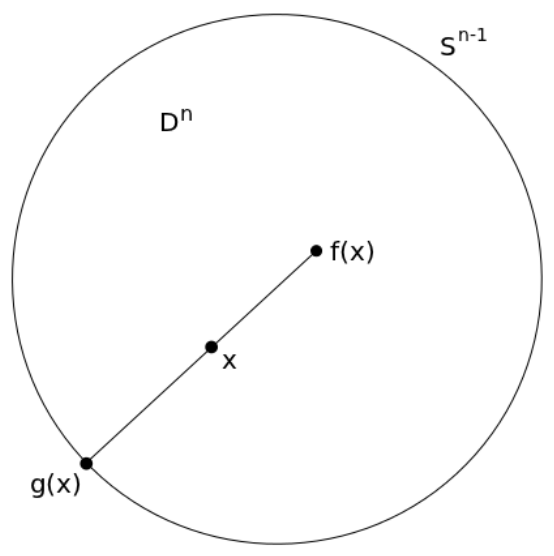

Temos que $g(x)=x+t u$, onde $u=\frac{x-f(x)}{\|x-f(x)\|}, \mathrm{e}$

$$
t=-x \cdot u+\sqrt{1-\|x\|^{2}+(x \cdot u)}
$$

Onde $x \cdot u$ é o produto interno usual. A expressão de $g(x)$ foi obtida da solução da equação $(x+t u) \cdot(x+t u)=1$. Temos duas soluções para esta equação, pois a semi-reta que liga $f(x)$ e $x$ e intercepta $S^{n-1}$ em dois pontos. Estamos interessados na solução com $t \geqslant 0$. Logo, $g$ é contínua com $\left.g\right|_{S^{n-1}}=\mathrm{id}_{S^{n-1}}$, o que é absurdo, pelo lema anterior.

O espaço tangente de $S^{n}$ em um ponto $x \in S^{n}$ é $T_{x} S^{n}=\{x\}^{\perp}$. Um campo vetorial tangente em $S^{n}$ é uma aplicação contínua $\nu: S^{n} \longrightarrow \mathbb{R}^{n+1}$ tal que $\nu(x) \in T_{x} S^{n}$ para todo $x \in S^{n}$.

Teorema 5.1.3. A esfera $S^{n}$ tem um campo vetorial tangente $\nu$ com $\nu(x) \neq 0$ para todo $x \in S^{n}$ se, e somente se, $n$ é impar.

Demonstração: $O$ campo vetorial $\nu$ pode ser estendido a um campo vetorial em $\mathbb{R}^{n}-\{0\}$ por:

$$
\omega(x)=\nu\left(\frac{x}{\|x\|}\right)
$$

Temos que $\omega(x) \neq 0$ e $\omega(x) \cdot x=0$. Tomemos a homotopia entre $f_{0}=\mathrm{id}_{\mathbb{R}^{n+1}-\{0\}}$ e a aplicação antipodal $f_{1}(x)=-x$, dada por:

$$
F(x, t)=(\cos \pi t) x+(\sin \pi t) \omega(x) .
$$


O item (i) do teorema 4.1.11 nos dá que $f_{1}^{*}$ é a identidade em $H^{n}\left(\mathbb{R}^{n+1}-\{0\}\right)$, que, pelo teorema 4.1.16 é 1-dimensional. Por outro lado o lema 4.1.18 nos diz que $f_{1}^{*}$ é a multiplicação por $(-1)^{n+1}$, logo, $n$ tem que ser ímpar.

Reciprocamente, para $n=2 m-1$, podemos definir um campo vetorial $\nu$ por:

$$
\nu\left(x_{1}, x_{2}, \ldots, x_{2 m}\right)=\left(x_{2},-x_{1}, x_{4},-x_{3}, \ldots, x_{2 m},-x_{2 m-1}\right) .
$$

Lema 5.1.4. (Urysohn-Tietze) $S e A \subseteq \mathbb{R}^{n}$ é fechado e $f: A \longrightarrow \mathbb{R}^{m}$ uma aplicação contínua, então existe uma aplicação contínua $g: \mathbb{R}^{n} \longrightarrow \mathbb{R}^{m}$ tal que $\left.g\right|_{A}=f$.

Demonstração: Denotaremos a distância euclidiana em $\mathbb{R}^{n}$ por $d(x, y)$ e definimos a distância entre um ponto e um conjunto $A$ por $d(x, A)=\inf _{y \in A} d(x, y)$.

Para $p \in \mathbb{R}^{n}-A$, temos a vizinhança aberta $U_{p} \subseteq \mathbb{R}^{n}-A$ de $p$ dada por:

$$
U_{p}=\left\{x \in \mathbb{R}^{n} \mid d(x, p)<\frac{1}{2} d(p, A)\right\}
$$

Essas vizinhanças dos $p \in \mathbb{R}^{n}-A$ fornecem uma cobertura aberta de $\mathbb{R}^{n}-A$. Usando o lema B do apêndice deste trabalho, podemos obter uma partição da unidade $\phi_{p}$ subordinada a cobertura aberta encontrada. Definimos a aplicação $g$ da seguinte forma:

$$
g(x)=\left\{\begin{array}{cll}
f(x) & \text { se } & x \in A \\
\sum_{p \in \mathbb{R}^{n}-A} \phi_{p}(x)(a(p)) & \text { se } & x \in \mathbb{R}^{n}-A
\end{array}\right.
$$

onde $a(p) \in A$ é escolhido de tal forma que $d(p, a(p))<2 d(p, A)$. Como a soma é localmente finita em $\mathbb{R}^{n}-A$, g é suave em $\mathbb{R}^{n}-A$. Resta mostrar a continuidade de $g$ para pontos $x_{0}$ na fronteira de $A$. Assim, se $x \in U_{p}$, então:

$$
d\left(x_{0}, p\right) \leqslant d\left(x_{0}, x\right)+d(x, p)<d\left(x_{0}, x\right)+\frac{1}{2} d(p, A) \leqslant d\left(x_{0}, x\right)+\frac{1}{2} d\left(p, x_{0}\right) .
$$

Então, $d\left(x_{0}, p\right)<2 d\left(x_{0}, x\right)$ para $x \in U_{p}$. Como $d(p, a(p))<2 d(p, A) \leqslant 2 d\left(x_{0}, p\right)$, conseguimos, para $x \in U_{p}$ que $d\left(x_{0}, a(p)\right) \leqslant d\left(x_{0}, p\right)+d(p, a(p))<3 d\left(x_{0}, p\right)<6 d\left(x_{0}, x\right)$. 
E, para todo $x \in \mathbb{R}^{n}-A$, temos:

$$
g(x)-g\left(x_{0}\right)=\sum_{p \in \mathbb{R}^{n}-A} \phi(x)_{p}\left(f(a(p))-f\left(x_{0}\right)\right)
$$

E assim:

$$
\left\|g(x)-g\left(x_{0}\right)\right\| \leqslant \sum_{p} \phi(x)_{p}\left\|f(a(p))-f\left(x_{0}\right)\right\| .
$$

Agora, para um arbitrário $\epsilon>0$, escolhemos $\delta>0$ tal que $\left\|f(y)-f\left(x_{0}\right)\right\|<\epsilon$ para todo $y \in A \operatorname{com} d\left(x_{0}, y\right)<6 \delta$. Se $x \in \mathbb{R}^{n}-A$ e $d\left(x, x_{0}\right)<\delta$, então para qualquer $x \in U_{p}$, temos $d\left(x_{0}, a(p)\right)<6 \delta$ e $\left\|f(a(p))-f\left(x_{0}\right)\right\|<\epsilon$. Segue de $(*)$ que:

$$
\left\|g(x)-g\left(x_{0}\right)\right\| \leqslant \sum_{p} \phi(x)_{p}\left\|f(a(p))-f\left(x_{0}\right)\right\|<\sum_{p} \phi(x)_{p} \cdot \epsilon=\epsilon
$$

e $g$ é contínua também na fronteira de $A$.

Observação 5.1.5. A prova apresentada acima também contínua válida, com pequenas modificações, quando $\mathbb{R}^{n}$ é substituído por um espaço métrico e $\mathbb{R}^{m}$ por um espaço vetorial topológico localmente convexo.

Lema 5.1.6. Sejam $A \subseteq \mathbb{R}^{n}$ e $B \subseteq \mathbb{R}^{m}$ dois conjuntos fechados e $\phi: A \longrightarrow B$ é um homeomorfismo. Então existe um homeomorfismo $h: \mathbb{R}^{m+n} \longrightarrow \mathbb{R}^{m+n}$ tal que:

$$
h\left(x, 0_{m}\right)=\left(0_{n}, \phi(x)\right),
$$

para todo $x \in A$.

Demonstração: Pela lema 5.1 .4 podemos estender $\phi$ para uma aplicação contínua $f_{1}$ : $\mathbb{R}^{n} \longrightarrow \mathbb{R}^{m}$ e definir o homeomorfismo $h_{1}: \mathbb{R}^{n} \times \mathbb{R}^{m} \longrightarrow \mathbb{R}^{n} \times \mathbb{R}^{m}$ :

$$
h_{1}(x, y)=\left(x, y+f_{1}(x)\right) .
$$

Assim $h_{1}^{-1}(x, y)=\left(x, y-f_{1}(x)\right)$. Analogamente, considerando $\phi^{-1}: B \longrightarrow A$, a inversa de $\phi$, podemos estender $\phi^{-1}$ a uma aplicação contínua $f_{2}: \mathbb{R}^{m} \longrightarrow \mathbb{R}^{n}$ e definir

$$
h_{2}: \mathbb{R}^{n} \times \mathbb{R}^{m} \longrightarrow \mathbb{R}^{n} \times \mathbb{R}^{m}
$$


por:

$$
h_{2}(x, y)=\left(x+f_{2}(y), y\right) \text {. }
$$

onde $h_{2}^{-1}(x, y)=\left(x-f_{2}(y), y\right)$. Definimos $h$ como $h=h_{2}^{-1} \circ h_{1}$ e, para todo $x \in A$, temos:

$$
\begin{aligned}
h\left(x, 0_{m}\right)=h_{2}^{-1} \circ h_{1}\left(x, 0_{m}\right) & =h_{2}^{-1}\left(x, f_{1}(x)\right)=h_{2}^{-1}(x, \phi(x))=\left(x-f_{2}(\phi(x)), \phi(x)\right) \\
& =\left(x-\phi^{-1}(\phi(x)), \phi(x)\right)=\left(0_{n}, \phi(x)\right) .
\end{aligned}
$$

Vamos identificar $\mathbb{R}^{n}$ com um subespaço de $\mathbb{R}^{n+m}$ da seguinte forma:

$$
(x_{1}, x_{2}, \ldots, x_{n}, \overbrace{0, \ldots, 0}^{\text {m vezes }})
$$

Corolário 5.1.7. Se $\phi: A \longrightarrow B$ é um homeomorfismo entre subconjuntos fechados do $\mathbb{R}^{n}$, então $\phi$ pode ser estendida a um homeomorfismo $\widetilde{\phi}: \mathbb{R}^{2 n} \longrightarrow \mathbb{R}^{2 n}$.

Demonstração: Consideremos $h$ como no lema anterior. Seja $H: \mathbb{R}^{n} \times \mathbb{R}^{n} \longrightarrow \mathbb{R}^{n} \times \mathbb{R}^{n}$, dada por $H(x, y)=(y, x)$ e $\widetilde{\phi}=H \circ h$.

Observação 5.1.8. A aplicação encontrada $\widetilde{\phi}$ nós dá também um homeomorfismo entre $\mathbb{R}^{2 n}-A$ e $\mathbb{R}^{2 n}-B$ por restrição.

Teorema 5.1.9. Tomemos $A \varsubsetneqq \mathbb{R}^{n}$ e $B \varsubsetneqq \mathbb{R}^{n}$ fechados. Se $A$ e $B$ são homeomorfos, então

$$
H^{p}\left(\mathbb{R}^{n}-A\right) \cong H^{p}\left(\mathbb{R}^{n}-B\right)
$$

Demonstração: Mostremos primeiramente, por indução sobre $m$, que:

$$
\begin{aligned}
H^{p+m}\left(\mathbb{R}^{m+n}-A\right) & \cong H^{p}\left(\mathbb{R}^{n}-A\right) \quad p>0 \\
H^{m}\left(\mathbb{R}^{m+n}-A\right) & \cong H^{0}\left(\mathbb{R}^{n}-A\right) / \mathbb{R} \cdot 1
\end{aligned}
$$

O caso $m=1$ é a proposição 4.1.14. Suponhamos verdadeiro para algum $k \geqslant 2$. Então:

$$
\begin{aligned}
H^{p+k}\left(\mathbb{R}^{k+n}-A\right) & \cong H^{p}\left(\mathbb{R}^{n}-A\right) \quad p>0 \\
H^{k}\left(\mathbb{R}^{k+n}-A\right) & \cong H^{0}\left(\mathbb{R}^{n}-A\right) / \mathbb{R} \cdot 1 .
\end{aligned}
$$

Pela proposição 4.1.14 e pela hipótese de indução

$$
H^{p+k+1}\left(\mathbb{R}^{n+k+1}-A\right) \cong H^{p+k}\left(\mathbb{R}^{n+k}-A\right) \cong H^{p}\left(\mathbb{R}^{n}-A\right) \cong H^{n+1}\left(\mathbb{R}^{k+1}-A\right)
$$


e

$$
H^{k+1}\left(\mathbb{R}^{n+k+1}-A\right) \cong H^{1}\left(\mathbb{R}^{n+1}-A\right) \cong H^{0}\left(\mathbb{R}^{n}-A\right) / \mathbb{R} \cdot 1
$$

Logo, (\#) é válida para todo $m \geqslant 0$. Analogamente, para $B$ temos:

$$
\begin{aligned}
H^{p+m}\left(\mathbb{R}^{m+n}-B\right) & \cong H^{p}\left(\mathbb{R}^{n}-B\right) \quad p>0 \\
H^{m}\left(\mathbb{R}^{m+n}-B\right) & \cong H^{0}\left(\mathbb{R}^{n}-B\right) / \mathbb{R} \cdot 1 .
\end{aligned}
$$

No corolário anterior mostramos que $\mathbb{R}^{2 n}-A$ e $\mathbb{R}^{2 n}-B$ são homeomorfos. O corolário de invariância topológica 4.1 .12 mostra que isso induz um isomorfismo entre suas cohomologias de de Rham. Assim, para $p>0$ :

$$
H^{p}\left(\mathbb{R}^{n}-A\right) \cong H^{p+n}\left(\mathbb{R}^{2 n}-A\right) \cong H^{p+n}\left(\mathbb{R}^{2 n}-B\right) \cong H^{p}\left(\mathbb{R}^{n}-B\right)
$$

e, $\operatorname{para} p=0$ :

$$
H^{0}\left(\mathbb{R}^{n}-A\right) / \mathbb{R} \cdot 1 \cong H^{n}\left(\mathbb{R}^{2 n}-A\right) \cong H^{n}\left(\mathbb{R}^{2 n}-B\right) \cong H^{0}\left(\mathbb{R}^{n}-B\right) / \mathbb{R} \cdot 1
$$

Observação 5.1.10. Para $A \subseteq \mathbb{R}^{n}$ fechado, o complementar $U=\mathbb{R}^{n}-A$ é um aberto $e$ será uma união disjunta e enumerável de componentes conexas, todas abertas. Se forem infinitas, então $\operatorname{dim}\left(H^{0}(U)\right)=\infty$. Caso contrário, o número de componentes conexas é igual a $\operatorname{dim}\left(H^{0}(U)\right)$.

Corolário 5.1.11. Se $A$ e $B$ são dois fechados homeomorfos em $\mathbb{R}^{n}$, então $\mathbb{R}^{n}-A$ e $\mathbb{R}^{n}-B$ tem o mesmo número de componentes conexas.

Demonstração: Se $A \neq \mathbb{R}^{n}$ e $B \neq \mathbb{R}^{n}$, então o resultado segue no teorema e da observação anteriores. Se $A=\mathbb{R}^{n}$ e $B \neq \mathbb{R}^{n}$, temos $\mathbb{R}^{n+1}-A$ com duas componentes conexas, enquanto $\mathbb{R}^{n+1}-B$ será conexo, o que não pode acontecer.

Teorema 5.1.12. (da separação de Jordan-Brouwer) $S e \Sigma \subseteq \mathbb{R}^{n}$, com $n \geqslant 2$ é homeomorfo a $S^{n-1}$ ent $\tilde{a} o$ :

(i) $\mathbb{R}^{n}-\Sigma$ tem exatamente duas componentes conexas $U_{1}$ e $U_{2}$, onde $U_{1}$ é limitada e $U_{2}$ é ilimitada; 
(ii) $\Sigma$ é a fronteira de $U_{1}$ e $U_{2}$.

Demonstração: Como $\Sigma$ é compacto, então é fechado em $\mathbb{R}^{n}$. Usando o corolário 5.1.11. temos o mesmo número de componentes conexas em $\mathbb{R}^{n}-\Sigma$ e em $\mathbb{R}^{n}-S^{n-1}$. Consideremos os conjuntos

$$
D^{\circ n}=\left\{x \in \mathbb{R}^{n} \mid\|x\|<1\right\} \quad \text { e } \quad W=\left\{x \in \mathbb{R}^{n} \mid\|x\|>1\right\} .
$$

Ambos conexos com $\mathbb{R}^{n}-S^{n-1}=D^{\circ n} \cup W$. Logo, $\mathbb{R}^{n}-S^{n-1}$ tem duas componentes conexas, e assim, $\mathbb{R}^{n}-\Sigma$ também tem.

Tomemos $r=\max _{x \in \Sigma}\|x\|, r<\infty$ pois $\Sigma$ é compacto e a norma é contínua. Consideremos

$$
r W=\left\{x \in \mathbb{R}^{n} \mid\|x\|>r\right\}
$$

Notemos que $r W \cap \Sigma=\varnothing$ e que $r W$ é conexo. Assim, devemos ter $r W$ inteiramente contido em uma das componente conexa de $\mathbb{R}^{n}-\Sigma$. Como $r W$ é ilimitado, está contido em $U_{2}$ e $U_{2}$ é ilimitada. Assim, $U_{1}$ deverá estar contido em $\mathbb{R}^{n}-r W=\left\{x \in \mathbb{R}^{n} \mid\|x\| \leqslant r\right\}$, logo, $U_{1}$ está contida em um conjunto limitado e, portanto, será limitada.

Para provar (ii), tomemos $p \in \Sigma$ e consideremos $V$ uma vizinhança aberta qualquer de $p$. Como $\Sigma$ é fechado e $V$ aberto, temos $A=\Sigma-(\Sigma \cap V)$ será fechado em $\Sigma$ e será homeomorfo a um conjunto fechado $B \subsetneq S^{n-1}$. Claro que $\mathbb{R}^{n}-B$ será conexo e, pelo corolário 5.1.11 temos $\mathbb{R}^{n}-A$ conexo. Assim, sejam $p_{1} \in U_{1}, p_{2} \in U_{2}$ e $\gamma:[a, b] \longrightarrow \mathbb{R}^{n}-A$ uma curva tal que $\gamma(a)=p_{1}$ e $\gamma(b)=p_{2}$. Por (i) temos que $\gamma$ intercepta $\Sigma$ pelo menos uma vez e $\gamma^{-1}[a, b] \subseteq[a, b]$ é um conjunto fechado. Consideremos $\gamma\left(c_{1}\right)$ e $\gamma\left(c_{2}\right)$ o primeiro e o último pontos de interseç̧ão de $\gamma \operatorname{com} \Sigma$. Como $\gamma(a)=p_{1}$ e $\gamma(b)=p_{2}$, temos que $c_{1}, c_{2} \in(a, b)$. Além disso $\gamma\left(c_{1}\right) \in \Sigma \cap V$ e $\gamma\left(c_{2}\right) \in \Sigma \cap V$ e mais, $\gamma\left(\left[a, c_{1}\right)\right) \subseteq U_{1}$ e $\gamma\left(\left(c_{2}, b\right]\right) \subseteq U_{2}$. Então, podemos encontrar $t_{1} \in\left[a, c_{1}\right)$ e $t_{2} \in\left(c_{2}, b\right]$ tais que $\gamma\left(t_{1}\right) \in U_{1} \cap V$ e $\gamma\left(t_{2}\right) \in U_{2} \cap V$, e assim $p$ será um ponto de fronteira.

Teorema 5.1.13. Se $A \subseteq \mathbb{R}^{n}$ é homeomorfo a $D^{k}$ com $k \leqslant n$, então $R^{n}-A$ é conexo.

Demonstração: $A$ é compacto e, portanto, fechado. Como temos $D^{k} \subseteq \mathbb{R}^{k} \subseteq \mathbb{R}^{n}$ e $R^{n}-D^{k}$ conexo, o corolário 5.1 .11 nos dá o resultado.

Teorema 5.1.14. (Brouwer) Seja $U \subseteq \mathbb{R}^{n}$ um aberto e $f: U \longrightarrow \mathbb{R}^{n}$ uma aplicação continua e injetiva. A imagem $f(U)$ é aberta em $\mathbb{R}^{n}$ e f aplica $U$ homeomorficamente em $f(U)$. 
Demonstração: É suficiente provar que $f(U)$ é aberto, pois isso fará com que $\mathrm{f}(\mathrm{W})$ seja aberto para $W \subseteq U$, e isso provará a continuidade de $f^{-1}: f(U) \longrightarrow U$, a inversa de $f$. Consideremos a bola fechada com centro em $x_{0} \in U$ :

$$
D=\left\{x \in \mathbb{R}^{n} \mid\left\|x-x_{0}\right\| \leqslant \delta\right\}
$$

Contida em $U$ e com fronteira $S$ e interior $D^{\circ}=D-S$. É suficiente mostrar que $f\left(D^{\circ}\right)$ é aberto. Quando $n=1$, temos $f\left(D^{\circ}\right)$ aberto, pois $f$ é contínua e função de uma variável.

Assumimos $n \geqslant 2$. Tanto $S$ quanto $\Sigma=f(S)$ são homeomorfos a $S^{n-1}$. Tomemos $U_{1}$ e $U_{2}$ as duas compomentes conexas de $\mathbb{R}^{n}-\Sigma$, como no teorema 5.1.12. As duas são abertas, $U_{1}$ é limitada e $U_{2}$ é ilimitada. Pelo teorema $5.1 .13 \mathbb{R}^{n}-f(D)$ é conexo e como $\left(\mathbb{R}^{n}-f(D)\right) \cap \Sigma=\varnothing$, devemos ter $\mathbb{R}^{n}-f(D) \subseteq U_{2}$ ou $\mathbb{R}^{n}-f(D) \subseteq U_{1}$, mas $\mathbb{R}^{n}-D$ é ilimitado, então $\mathbb{R}^{n}-f(D) \subseteq U_{2}$. Assim $\Sigma \cup U_{1}=\mathbb{R}^{n}-U_{2} \subseteq f(D)$. Então $U_{1} \subseteq f\left(D^{\circ}\right)$. Como $D^{\circ}$ é convexo, $f\left(D^{\circ}\right)$ também é conexo, além disso $f\left(D^{\circ}\right) \subseteq U_{1} \cup U_{2}$, então $f\left(D^{\circ}\right)=U_{1}$, ou seja, $f\left(D^{\circ}\right)$ é aberto.

Corolário 5.1.15. (Invariância de dominio) $S e V \subseteq \mathbb{R}^{n}$ tem a topologia induzida do $\mathbb{R}^{n}$ e é homeomorfo a um aberto de $\mathbb{R}^{n}$, então $V$ é um aberto de $\mathbb{R}^{n}$.

Demonstração: Ora, sendo $V$ homeomorfo a algum aberto $U \subseteq \mathbb{R}^{n}$ por alguma aplicação $f$, então $V=f(U)$ e o resultado segue do teorema anterior.

Corolário 5.1.16. (Invariância da dimensão) Sejam $U \subseteq \mathbb{R}^{n}$ e $V \subseteq \mathbb{R}^{m}$ dois abertos não vazios. Se $U$ é homeomorfo a $V$ então $m=n$.

Demonstração: Assumimos que $m<n$. Do corolário anterior aplicado a $V$, considerado como um subconjunto de $\mathbb{R}^{n}$ pela inclusão $\mathbb{R}^{m} \subset \mathbb{R}^{n}$, segue que $V$ é aberto em $\mathbb{R}^{n}$, o que não é possível, pois $V$ está contido em um subespaço próprio.

Exemplo 5.1.17. Um nó em $\mathbb{R}^{3}$ é um subconjunto $\Sigma \subseteq \mathbb{R}^{3}$ que é homeomorfomo a $S^{1}$. O nó complementar, é um aberto $U=\mathbb{R}^{3}-\Sigma$. Mostremos que:

$$
H^{p}(U) \cong \begin{cases}\mathbb{R} & \text { se } 0 \leqslant p \leqslant 2 \\ 0 & \text { caso contrário. }\end{cases}
$$

De acordo com o teorema 5.1 .9 é suficiente mostrar isso com o nó trivial $S^{1} \subseteq \mathbb{R}^{2} \subseteq$ 
$\mathbb{R}^{3}$. Calculemos:

$$
H^{p}\left(\mathbb{R}^{2}-S^{1}\right)=H^{p}\left(D^{\circ 2}\right) \oplus H^{p}\left(\mathbb{R}^{2}-D^{2}\right)
$$

Onde $D^{\circ 2}$ é o interior de $D^{2}$. Temos que $D^{\circ 2}$ é estrelado $e \mathbb{R}^{2}-D^{2}$ difeomorfo a $\mathbb{R}^{2}-\{0\}$. Usando o teorema 1.3.2 e o exemplo 3.0 .13 chegamos a conclusão de que * tem dimensão 2 para $p=0$, dimensão 1 para $p=1$ e dimensão 0 para $p \geqslant 2$. Aplicando a proposição 4.1.14, temos que $H^{p+1}\left(\mathbb{R}^{3}-S^{1}\right) \cong H^{p}\left(\mathbb{R}^{2}-S^{1}\right)$. 


\section{Anexo}

\section{Lema A}

Suponhamos que $A \subseteq U_{0} \subseteq U \subseteq \mathbb{R}^{n}$, onde $U_{0}$ e $U$ são abertos do $\mathbb{R}^{n}$ e $A$ fechado em $U$. Seja $h: U \longrightarrow W$ uma aplicação contínua em um aberto $W \subseteq \mathbb{R}^{m}$, com restrição suave à $U_{0}$. Para qualquer função contínua $\epsilon: U \longrightarrow(0, \infty)$ existe uma função suave $f: U \longrightarrow W$ que satisfaz:

(i) $\|f(x)-h(x)\| \leqslant \epsilon(x)$, para todo $x \in U$;

(ii) $f(x)=h(x)$, para todo $x \in A$.

\section{Lema B (Partição da Unidade)}

Se $U \subseteq \mathbb{R}^{n}$ é um aberto e $V=\left(V_{i}\right)_{i \in I}$ uma cobertura de $U$ onde $V_{i}$ são abertos, então existem funções suaves $\phi_{i}: U \longrightarrow[0,1] \operatorname{com} i \in I$, satisfazendo:

(i) $\operatorname{supp}_{U}\left(\phi_{i}\right) \subseteq V_{i}$ para todo $i \in I$;

(ii) Todo $x \in U$ tem uma vizinhança $W$ onde apenas um número finito de $\phi_{i}$ não são nulas;

(iii) Para todo $x \in U$ temos $\sum_{i \in I} \phi_{i}(x)=1$.

Ou seja, $\left(\phi_{i}\right)_{i \in I}$ é uma partição da unidade formada por funções suaves e subordinada a cobertura $V$. 


\section{Referências Bibliográficas}

[1] Lima, E.L., Álgebra Exterior Coleção Matemática Universitária, Rio de Janeiro, IMPA, 2005.

[2] Lima, E.L., Curso de Análise Volume 2 Rio de Janeiro, IMPA, 1981.

[3] Lima, E.L., Análise Real Volume 3 Coleção Matemática Universitária, Rio de Janeiro, IMPA, 2008.

[4] Lima, E.L., Homologia Básica, Rio de Janeiro, IMPA, 2009.

[5] Madsen, I. H., From calculus to cohomology: de Rham cohomology and characteristic classes, Cambrigde University Press, 1996. 